Shedding light on fractals:

Exploration of the

Sierpinski carpet optical antenna

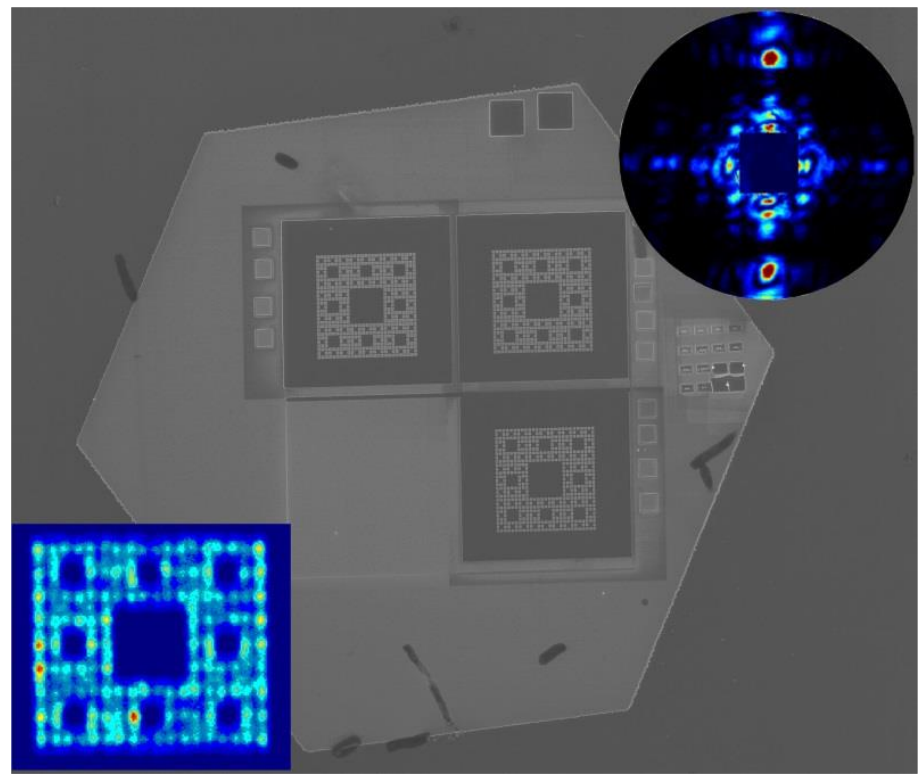

Ting Lee Chen 
SHEDDING LIGHT ON FRACTALS:

EXPLORATION OF THE

\section{SIERPINSKI CARPET OPTICAL ANTENNA}


Thesis committee members

$\begin{array}{lll}\text { Supervisor } & \text { Prof. dr. J. L. Herek } & \text { Universiteit Twente } \\ & & \\ \text { Invited members } & \text { Prof. dr. Rudolf Bratschitsch } & \text { Münster University } \\ & \text { Prof. dr. L. Kuipers } & \text { Utrecht University } \\ & \text { Prof. dr. K. J. Boller } & \text { Universiteit Twente } \\ & \text { Prof. dr. H. Gardiniers } & \text { Universiteit Twente } \\ & \text { dr. Ir. M. J. Bentum } & \text { Universiteit Twente }\end{array}$

This work was carried out at the Optical Sciences group, which is a part of:

Department of Science and Technology

and MESA+ Institute for Nanotechnology,

University of Twente, P.O. Box 217,

7500 AE Enschede, The Netherlands.

Financial support was provided by a VICI grant from the CW section of the Nederlandse Wetenschappelijk Organisatie (NWO) to Prof. Jennifer L. Herek.

ISBN: 978-90-365-3894-7

\section{Copyright $@ 2015$ by Ting Lee Chen}

All rights reserved. No part of the material protected by this copyright notice may be reproduced or utilized in any form or by any means, electronic or mechanical, including photocopying, recording or by any information storage and retrieval system, without the prior permission of the author. 


\title{
SHEDDING LIGHT ON FRACTALS: EXPLORATION OF THE SIERPINSKI CARPET OPTICAL ANTENNA
}

\section{DISSERTATION}

\author{
to obtain \\ the degree of doctor at the University of Twente, \\ on the authority of the rector magnificus, \\ Prof. dr. H. Brinksma, \\ on account of the decision of the graduation committee, \\ to be publicly defended \\ on Thursday 21 May 2015 at 16.45 \\ by \\ Ting Lee Chen \\ born on September 4, 1976 \\ in Hualien, Taiwan
}


This manuscript has been approved by:

Supervisor: Prof. dr. J. L. Herek 
To Jesus the Nazareth 



\section{Contents}

Chapter 1 Introduction 1

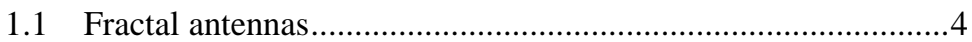

1.2 Surface plasmon polaritons and optical antennas .....................6

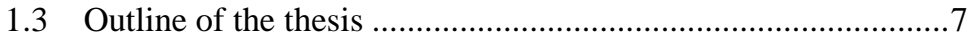

Chapter 2 The fundamental concepts 9

2.1 Concept of SPPs and LSPs...................................................10

2.1.1 Dielectric constant of metal: Drude model ..................10

2.1.2 The excitation of SPPs................................................12

2.1.3 The dispersion relation of SPP ..................................13

2.1.4 Localized surface plasmon (LSP) ...............................16

2.1.5 Localized surface plasmon resonance (LSPR) ............17

2.2 Introduction of optical antennas ............................................ 18

2.2.1 The bright and dark plasmonic mode ..........................19

2.2.2 The Fano resonance in optical antennas .....................21

Chapter 3 Sample fabrication and characterization methodologies 27

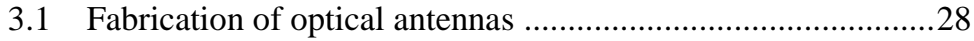

3.1.1 Synthesis of single-crystalline gold flakes .................28

3.1.2 Removing residual chemicals on a gold flake by $\mathrm{O}_{2}$ plasma cleaning ............................................................. 30

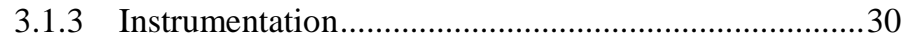

3.1.4 Fabrication result of the multi- crystalline gold film and single-crystalline gold flakes ...................................32

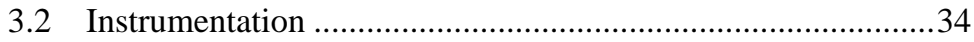


3.2.1 White light dark-field microscopy

3.2.2 Two-Photon Photoluminescence (TPPL)

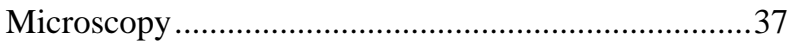

3.2.3 Back focal plane (leakage radiation) microscopy .........39

Chapter 4 Analysis of eigenmodes by eigen-decomposition method 43

4.1 The eigen-decomposition theory of plasmonic resonances .....44

4.1.1 The coupled dipole equation.........................................44

4.1.2 The concept of eigenpolarizability …...........................45

4.1.3 Localization of plasmonic eigenmode ........................46

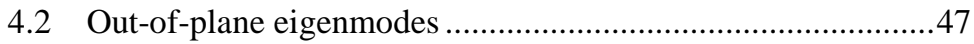

4.2.1 The eigenpolarizability spectra of out-of-plane eigenmodes ............................................................... 47

4.2.2 The eigenmodes of superradiant peak ........................50

4.2.3 The eigenmodes of subradiant peak ….........................51

4.3 In-plane eigenmodes ............................................................54

4.3.1 The eigenpolaribility spectra of in-plane

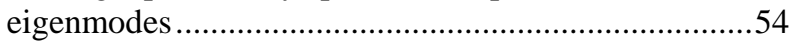

4.3.2 The self-similarity in the structure of dipole

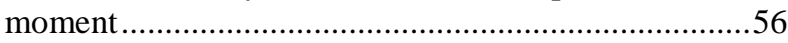

4.4 The trajectory plot of wavelength - far field radiation

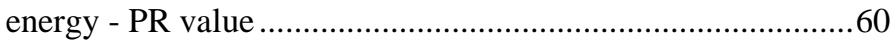

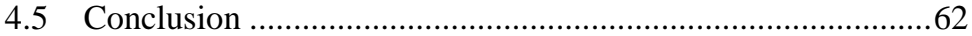

Chapter 5 Characterization at visible and near-infrared wavelengths 63

5.1 Three morphologies of broadband optical antennas................64

5.1.1 Sample schematics......................................................64

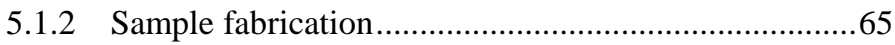

5.2 Far-field scattering property ...............................................67 
5.3 Near-field intensity measurements .68

5.3.1 The two photon photoluminescence images of samples

5.3.2 The estimation of near field intensity by TPPL images.

5.3.3 The FDTD simulation result of TPPL enhancement factor $\alpha$

5.4 Conclusion .75

Chapter 6 Observation of surface lattice resonance

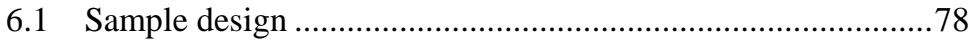

6.2 The FDTD method and coupled dipole approximation ............79

6.3 Comparison of diffraction images with calculated results ......80

6.4 The dispersion relation from BFP images ................................ 84

6.5 The calculation of dominate eigenmode by eigen-

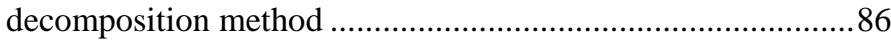

6.6 The Fano-dip in scattering spectrum ...................................... 88

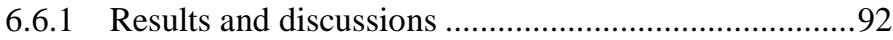

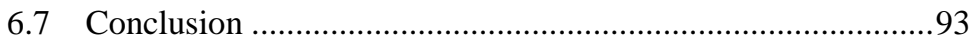

Chapter 7 Outlook and applications 95

7.1 The investigations of other aperiodic or fractal metal nanoparticle arrays .............................................................. 96

7.2 The potential application of Sierpinski carpet optical

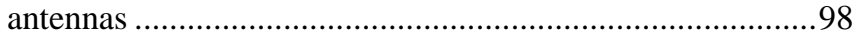

$\begin{array}{ll}\text { Bibliography } & 101\end{array}$

List of publications 115

$\begin{array}{ll}\text { Summary } & 117\end{array}$

$\begin{array}{ll}\text { Acknowledgements } & 121\end{array}$ 



\section{Chapter 1}

\section{Introduction}

Since Marconi began his pioneering work on signal transmission through wireless systems in 1895 [1], antennas have revolutionized the way of transferring messages and information among people. Nowadays antennas are widely used in radio, television, radar, mobile phones and satellite communications. The function of an antenna is to capture or emit electromagnetic waves. These may be propagating through the air, under the water, in outer space beyond the atmosphere of the earth, or even inside of soil and rocks. Antennas are constructed of conductive metals, and the specific design - corresponding to the working wavelength region-depends on the purpose. The dipole, bowtie or Yagi-Uda design of antennas [2] are very common and can be seen everywhere (Fig. 1.1). A simple design rule, for example for the dipole antenna, is that the length of the antenna is approximately half of the operating wavelength.

(a)

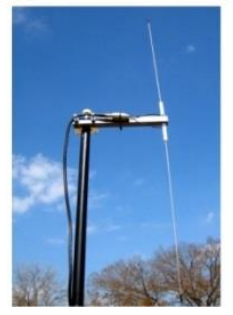

(b)

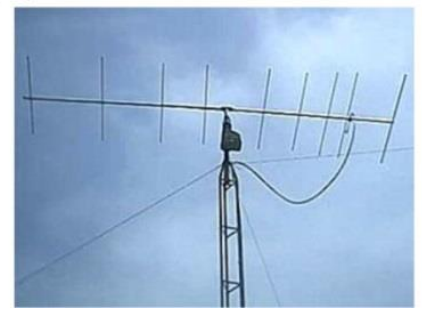

(c)

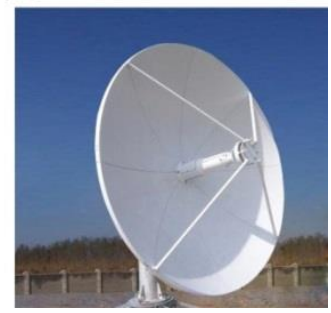

Fig. 1.1: Different antennas for various applications. (a) A long range VHF wireless microphone $1 / 2$ wave dipole antenna (adaption from FM DX Antenna). It works at $150 \mathrm{MHz}$ through 216 MHz. (b) A Yagi-Uda antenna. It operates in the HF to UHF bands. (about $3 \mathrm{MHz}$ to $3 \mathrm{GHz}$ ) (c) A microwave satellite antenna (adaption from Probecom). It operates at 12.5 to $12.75 \mathrm{GHz}$ 
Less common but arguably more interesting antenna designs are based on fractal geometries. The fractal geometry is built upon the rule of selfsimilarity, in which a characteristic pattern repeats itself on different length scales, which is called 'scale invariance'. The history of fractals started already in the 17th century, when Leibniz considered self-similarity in his writing [3]. Following the advent of the computer age, in the 1970s Mandelbrot introduced the word "fractal" and illustrated the concept with stunning computer-aided graphics [4].

Fractals also exist in nature. Fig. 1.2 shows examples of natural objects exhibiting self-similar patterns: a Romanesco broccoli, frost crystals and an autumn fern. The same patterns in each of them can approximately exist at different scales. It means that if we zoom in a smaller portion of a fractal object, it still shows the representative features of the whole object.

(a)

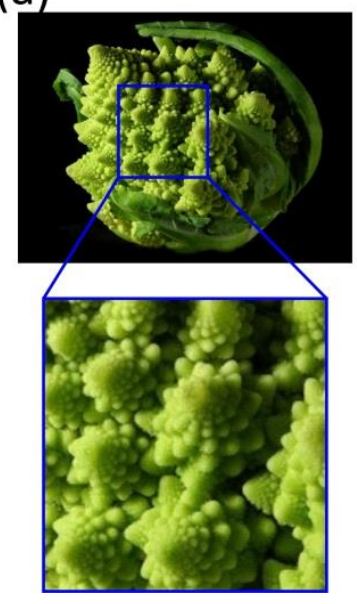

(b)

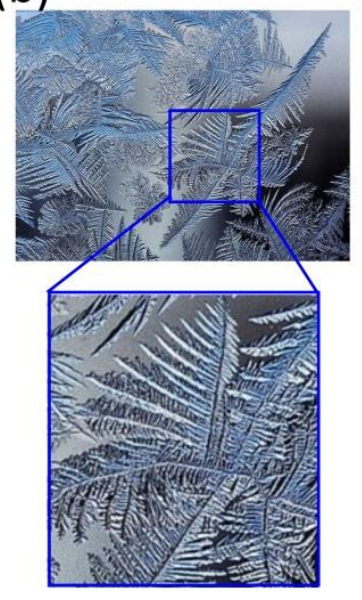

(c)

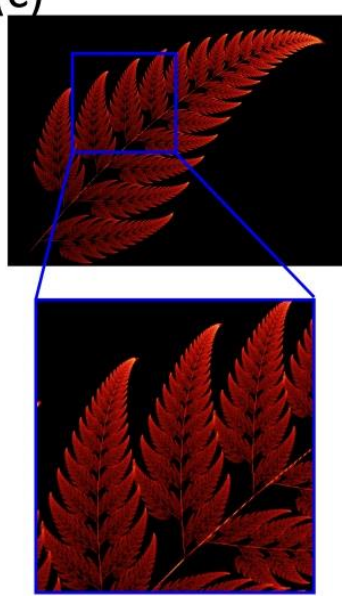

Fig. 1.2: Examples of the fractal geometry in natural objects. (a) the Romanesco broccoli. (b) frost crystals on a glass. (c) an autumn fern. The self-similarity of each object can be seen from their magnification of detailed structures within the blue frame.

Another fractal example having a rigorous mathematical definition is the Sierpinski carpet. In 1916, Wacław Sierpiński described a fractal geometry 
by subdividing a shape into smaller copies of itself, removing one or more copies, and continuing recursively [5]. This process can be used on any shape such as an equilateral triangle or square. Fig. 1.3 shows the construction process for a square - the creation of the Sierpinski carpet. The square is cut into 9 congruent subsquares in a 3-by-3 grid, with the central subsquare removed. The same procedure is then applied recursively to the remaining 8 subsquares. We can calculate the area of Sierpinski carpet in each iteration. Assuming the area of square in (a) is 1 , then the area of Sierpinski carpet (b), (c) and (d) are $8 / 9,(8 / 9)^{2}$ and $(8 / 9)^{3}$. If we continue this iteration to infinity, we get the area $(8 / 9)^{\infty}=0$. Therefore, we see that the scale invariance property of Sierpinski carpet gives an odd result that the area becomes zero. We may ask another question about how big the Sierpinski carpet is, or more precisely: what is the dimension of a fractal? This question involves the concept of 'fractal dimension' which is not discussed here. The dimension for the Sierpinski carpet is not a positive integer but equals $\ln 8 / \ln 3 \approx 1.8928$ [6].

(a)

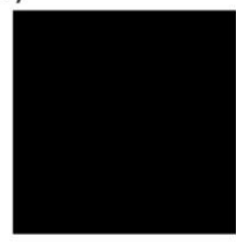

(b)

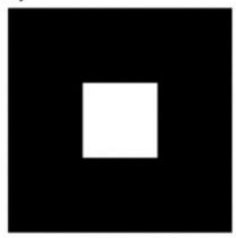

(c)

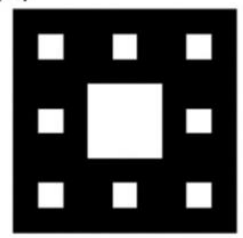

(d)

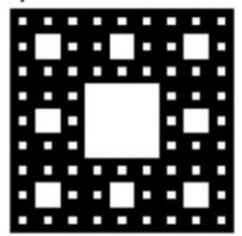

Fig. 1.3: The construction of a Sierpinski carpet. Starting from $0^{\text {th }}$ order (a), a solid square is divided into a $3 \times 3$ grid congruent subsquares and the center one is removed (b). The same procedure is applied to each subsquare, then each subsquare (c), (d), etc.

Borrowing the idea of fractal antennas in radio frequency, this thesis explores the use of fractals in optical antennas. Among many different fractals, the 'Sierpinski carpet-like [6]' geometry shown in Fig. 1.4 has been chosen as a prototype system. We chose to start from a circle (or square) as the basic element. This circle is copied into an array of three by three and the central one is removed, creating the 1 st order unit of the structure. The same procedure is applied to construct the next order of the fractal pattern. With the continuation of this simple iterative process, for which the first three steps 
are visualized, this Sierpinski carpet inspired structure shows an increasing complexity in periodicity. Note that an essential difference between the structures we study here and the classical Sierpinski carpet is that the latter consists of one continuous material with holes (Fig. 1.3), whereas our design is constructed from isolated monomers arranged in the characteristic pattern. Nevertheless, the clear similarity merits the labeling of our structure as a Sierpinski carpet antenna, and the self-similarity character adopted in our Sierpinski carpet optical antennas, in which the monomer is a gold particle, will be studied and characterized by optical simulation methods as well as experimental tools.

Below we will introduce fractal antennas in the radio-frequency (RF) region, which provides the inspiration to pursue fractal optical antennas, as well as surface plasmon polaritons: an important research area that makes optical antennas feasible.
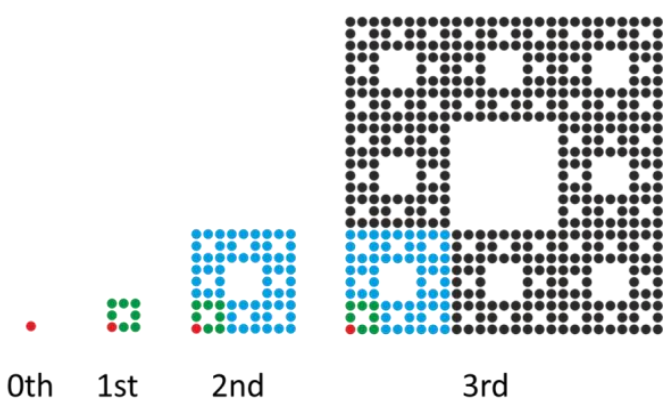

Fig. 1.4: The Sierpinski carpet inspired fractal structure that is investigated in this thesis. Starting from $0^{\text {th }}$ order - a monomer (red circle), then a $3 \times 3$ grid with the central monomer removed $\left(1^{\text {st }}\right.$ order) and so forth ( $2^{\text {nd }}$ and $3^{\text {rd }}$ order $)$.

\subsection{Fractal antennas}

In 1990, the concept of self-similarity was first introduced into the design of antennas for wireless communication [7]. Since then, various types of fractal geometries have been adapted to the design of antenna elements. Fractal antennas have been developed, patented and commercialized in mobile handsets [8] and radio-frequency identification readers [9]; see Fig. 1.5(a), 
(b). The key advantage of the fractal geometry is that it is compact in size yet capable of operating over a broadband response in the radio or microwave wavelength regimes.

(a)

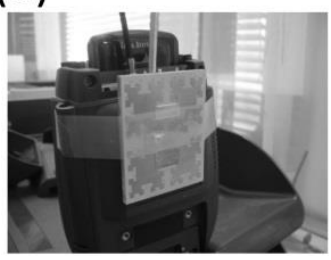

(b)

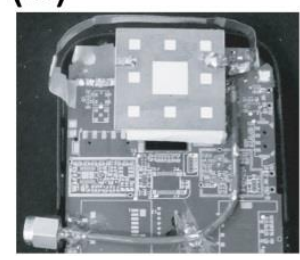

(c)

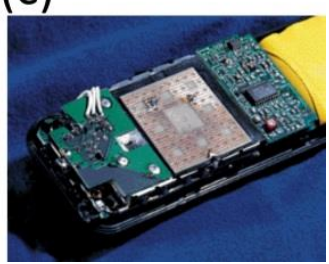

Fig. 1.5: (a) A fractal patch antenna attached to a handheld reader for performance testing (adapted from [8]). (b) A fractal antenna mounted on a candy bar phone(adapted from [9]). (c) A Sierpinski carpet fractal antenna in a cell phone. This image adapted from Ref. [10].

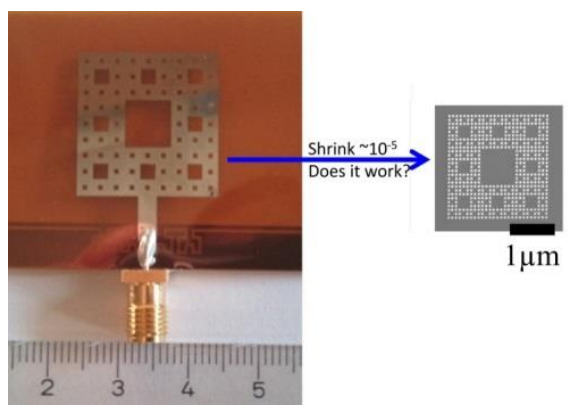

Fig. 1.6: Shrinking the size of fractal antenna to $\sim 10^{-5}$ scale. Left Sierpinski carpet fractal antenna is adapted from [12]. The right one is the SEM picture of a Sierpinski carpet optical antenna made by gold nanoparticles.

For world wide mobile service, there are at least five frequency bands currently assigned: 850, 900, 1800, 1900 and $2100 \mathrm{MHz}$. The dual bands for a mobile phone refers that it operates at $850 / 1800 \mathrm{MHz}$ frequencies in Europe or $850 / 1900 \mathrm{MHz}$ frequencies in north America. And the tri-band means that the operation frequencies are at 850/1700/1900 for North America or 850/1900/2100 for Europe and Asia. Many fractal antennas are thus designed for efficient broadband response at frequency regions of $824-960$ 
$\mathrm{MHz}$ and $1710-2170 \mathrm{MHz}$, corresponding to wavelengths of $0.31-0.36 \mathrm{~m}$ and $0.14-0.18 \mathrm{~m}$. It's not an exaggeration to say that today fractal antennas are hidden everywhere [10] [11] (e.g., Fig. 1.5(c)).

It is intriguing to consider what happens if we shrink the size of the Sierpinski carpet fractal antenna (see Fig. 1.6); can we make it active in the visible region simply by scaling? The visible light wavelengths range from $390-700 \mathrm{~nm}$, corresponding to $430-790 \mathrm{THz}$. The $10^{-5}$ scale difference between the wavelength of radio frequency and visible light presents a formidable challenge for the fabrication of fractal antennas for optical wavelength regions. This thesis describes the efforts of extending the concept of fractal geometry in antennas design to the visible and near-infrared wavelength regions.

\subsection{Surface plasmon polaritons and optical antennas}

Surface plasmon polaritons (SPPs) in metal nanoparticles have been found to be capable of enhancing light-matter interactions on the nanoscale, giving rise to a new research field called "plasmonics" [13]. Briefly speaking, the SPP is a collective motion of conduction electrons in metal films (propagating surface plasmon) or particles (localized surface plasmon) with the electromagnetic field of an incident light (Fig. 1.7(a)).

The rising interest and importance of the field of plasmonics is reflected in the amount of publications from 1990 to 2011, as shown in Fig. 1.7(b). After the year 2000, a steep rise is seen, corresponding to the proposal for using a metal thin film to create a "perfect lens" [16]. The negative refractive index of thin metal films was found to be able to counterintuitively enhance the amplitude of evanescent waves without the violation of the law of energy conservation. Therefore, a thin metal film can be used to focus a quasielectrostatic field beyond diffraction limit. It was also discovered that the condition of this focusing action of a metal film is exactly the same condition for the excitation of SPPs [17]. This finding has inspired many more researchers to explore SPPs and their applications. 
SPPs in metal nanoparticles confine and localize the electromagnetic energy of an incident light within several tens of nanometers, which is smaller than the wavelength of the incident light [13]. This property renders the enhancement of near field intensity and the increase of scattering cross section of metal nanoparticles, which favors the light-matter interaction of many nanophotonic devices [18] [19]. SPPs enable the development of optical antennas, and give rise to a different consideration of antenna designs for optical-frequency region from the RF region. It's important to understand the physics of SPPs in metals when we shrink the size of fractal antennas for the RF region to adapt them for the optical-frequency region. Further details will be discussed in Chapter 2.

(a)
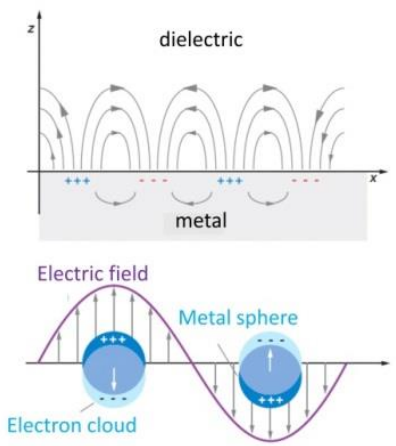

(b)

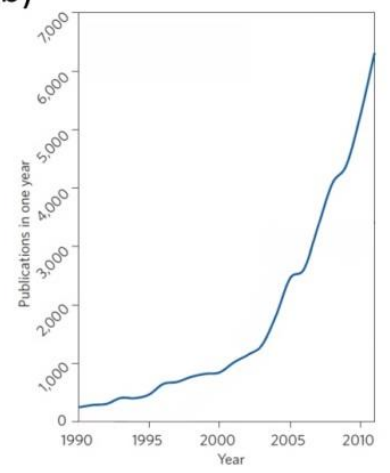

Fig. 17: (a) Upper: a surface plasmon polariton (or propagating surface plasmon). Lower: a localized surface plasmon. The figure is adapted from [14] (b) The comparison of publications of plasmons from year 1990 to 2011. The figure is adapted from [15].

\subsection{Outline of the thesis}

The central aim of this thesis is to elucidate the optical properties of fractal optical antennas. The focus here is on one type of fractal geometry-the Sierpinski carpet optical antenna-that serves as a rich playground for experimental and theoretical investigations. 
In Chaper 2, the fundamental concepts of surface plasmon polaritons (SPPs), localized surface plasmons (LSPs), and optical antennas are introduced. Optical antennas exploit SPPs as light localizers. The concepts of super- and sub- radiant modes of optical antennas are introduced. The Fano resonance caused by the interference between sub- and superradiant modes in optical antennas is introduced.

In Chapter 3, the sample fabrication including the instrument and materials are described. The schematics and concepts of the experimental setups used this research are presented, including. white-light dark field microscopy, two-photon photoluminescence microscopy and back focal plane microscopy.

In Chapter 4, the eigenmodes of the Sierpinski carpet optical antennas from $0^{\text {th }}-3^{\text {rd }}$ order are calculated by the eigen-decomposition method. The concept of eigenpolarizability is introduced. The dipole moment structure, the super- or sub-radiance and localization property of some representative eigenmodes are investigated. The self-similarity in the Sierpinski carpet optical antenna is correlated to the self-similarity in the dipole moment structure of some of the eigenmodes.

In Chapter 5, the far-field scattering and near-field intensity enhancement of three antenna morphologies - the Sierpinski carpet, the pseudo-random and periodic patterns-are investigated. Two-photon photoluminescence microscopy is adopted to measure the near-field intensity enhancement.

In Chapter 6, a surface lattice resonance of the Sierpinski carpet optical antenna is visualized by the back focal plane microscopy. The surface lattice resonance arises from the coupling a subradiant eigenmode with the in-plane diffracted light waves. This is the first time the resonant Wood's anomaly is visualized for an aperiodic fractal optical antenna.

Finally, in Chapter 7, the key results of this research are extracted, upon which an outlook for further work and potential applications is presented. 


\section{Chapter 2}

\section{The fundamental concepts}

This chapter introduces the fundamental concepts of surface plasmon polaritons (SPPs), localized surface plasmons (LSPs) and optical antennas, paving the way for understanding subsequent chapters in this thesis. The dispersion relation of SPPs is calculated, and the concepts of the Drude model and localized surface plasmon resonances are introduced. Super- and sub-radiant modes and Fano resonances of optical antennas are also introduced in this chapter. 


\subsection{Concept of SPPs and LSPs}

The diffraction limit was first derived by Ernst Abbe in 1873 [20], who determined that the size of the smallest spot in which light can be concentrated is around half of its wavelength. Recently it has been found that plasmons in metals can concentrate light beyond the diffraction limit [17]. A plasmon is a quantum of conduction electrons oscillating in a metal (Fig. 1.7(a)), which provides the nanoscale confinement and localization of light. This can hardly be found in other systems of such small scale and can be applied in almost every aspect of photonics [21]. In this section, we will introduce the Drude model which describes the motion of conduction electrons driven by an external electric field. This helps us to understand the resonance condition of LSPs. The excitation and dispersion relation of SPPs will be introduced. This section serves as preparation knowledge for understanding optical antennas introduced in the next section.

\subsubsection{Dielectric constant of metal: Drude model}

The Drude model is used for describing the behavior of valence electrons in a crystal structure of metals. The valence electrons are assumed to be completely detached from ions and form an electron gas. When light is incident on a metal, the electron gas oscillates and moves at a distance $x$ with respect to the positive ions, which causes the metal to be polarized with the surface charge density -nex, which produces a electric field $E=\frac{n e x}{\epsilon_{0}}$, where $n$ is the number of electrons; $e$ is the electric charge. The electric displacement $D(\omega)=\epsilon_{0} E(\omega)+P(\omega)$ and $D(\omega)=\epsilon_{0} \epsilon E(\omega)$, we have the dielectric constant:

$$
\epsilon(\omega)=1+\frac{|P(\omega)|}{\epsilon_{0}|E(\omega)|} .
$$

Here $\epsilon(\omega)$ is complex. The equation of motion for the electron gas can be written as:

$$
m \frac{d^{2} x}{d t^{2}}+m \gamma \frac{d x}{d t}=e E_{0} e^{-i \omega t}
$$


where $m$ is the effective mass of free electrons, $\gamma$ is the damping term and $\gamma=\frac{v_{F}}{l}, v_{F}$ is the Fermi velocity and 1 is the electron mean free path between scattering events.

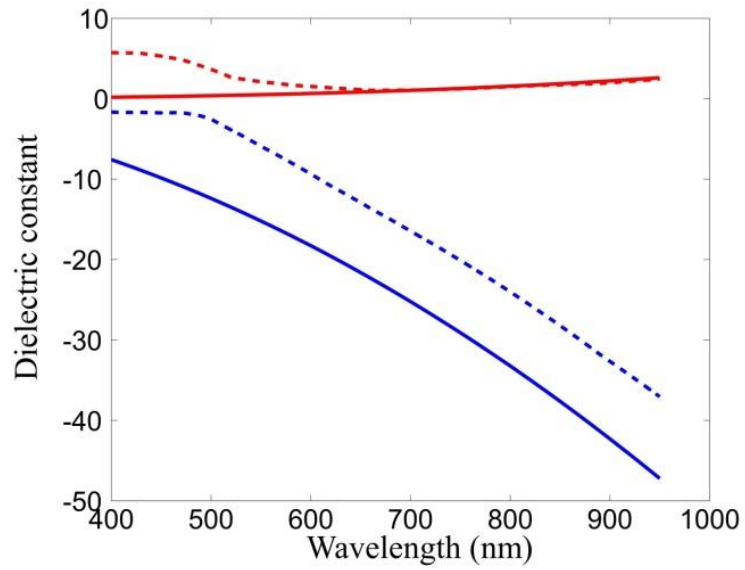

Fig. 2.1: The dielectric constant of gold calculated by the Drude model. The blue and red solid curves are the real and imaginary parts of the dielectric constant calculated by the Drude model, respectively. The blue and red dashed curves are the real and imaginary parts of the dielectric constant obtained from experimental data [22].

Solving Eq. 2.2 and using Eq. 2.1, the dielectric constant is:

$$
\epsilon_{\text {Drude }}(\omega)=1-\frac{\omega_{P}^{2}}{\omega^{2}+i \gamma \omega},
$$

where $\omega_{P}=\sqrt{\frac{n e^{2}}{m \epsilon_{0}}}$ is the plasma frequency.

For gold $\omega_{P}=13.8 \times 10^{15} \mathrm{~Hz}$ and $\gamma=1.075 \times 10^{14} \mathrm{~Hz}$; the real and imaginary parts of the Drude dielectric constant for gold is plotted in Fig. 2.1. We can see that the trend of the real (blue) and imaginary (red) parts of the dielectric constant calculated by the Drude model are similar to that of the experimental data [22]. For wavelengths below $550 \mathrm{~nm}$, the imaginary part of the dielectric function calculated by the Drude model is lower than the result 
of experiment data. This discrepancy is because the interband transitions are not considered in Drude model: the electrons in lower-lying bands absorb light photons with higher energies and transit to the conduction bands [23]. We should note that the real part of $\epsilon_{\text {Drude }}(\omega)$ is negative. This is an important feature for plasmonic materials, which determines the resonance condition of localized surface plasmons which will be introduced in Section 2.1.5.

We can further use the dielectric constant calculated by the Drude model to get the refractive index of metal. Let $N$ be the refractive index of metal, $N$ $=N_{r}+i N_{i}$, where $N_{r}$ is the real part and $N_{i}$ is the imaginary part of $N$. We have $\epsilon_{\text {Drude }}(\omega)=\epsilon^{\prime}+i \epsilon^{\prime \prime}=\left(N_{r}+i N_{i}\right)^{2}$, therefore,

$$
N_{r}=\sqrt{\frac{\sqrt{\epsilon^{\prime 2}+\epsilon^{\prime \prime^{2}}+\epsilon^{\prime}}}{2}} \text { and } N_{i}=\sqrt{\frac{\sqrt{\epsilon^{2}+\epsilon^{\prime \prime^{2}}}-\epsilon^{\prime}}{2}} \text {. }
$$

\subsubsection{The excitation of SPPs}

(a)

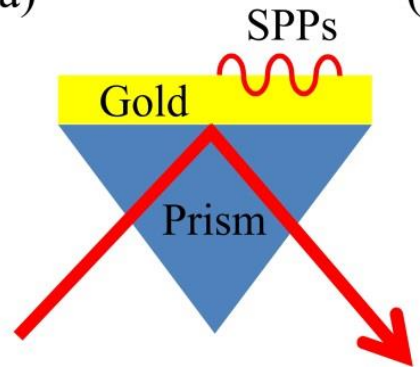

(b)

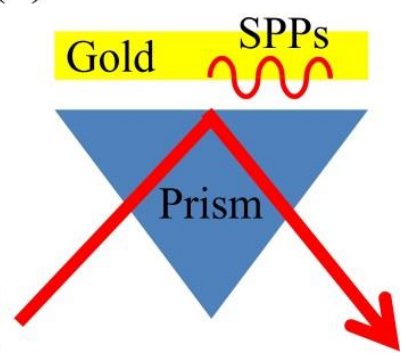

Fig. 2.2: (a) the Kretchmann configuration. (b) the Otto configuration.

Surface Plasmon Polaritons (SPPs) are longitundinal electromagnetic waves that propagate along the boundary between a metal and a dielectric layer. When SPPs are excited in a metal film, the collective motion of conduction electrons moves coherently with an incident light. The coherent motion between conduction electrons and light can be established only under the phase matching condition - the momentum of incident light waves matches 
the momentum of conduction electrons of a metal film. However, electrons have mass while photons do not; hence a special optical design is needed to excite the SPPs.

To meet the phase matching condition, optical designs such as Kretchmann and Otto configurations, named after their originators, are needed. Fig. 2.2(a), (b) show these two configurations [17]. In (a), a metal film (e.g. gold or silver) with a thickness of several tens of nanometer is coated on top of a prism. The excitation light is incident on the film from the prism side. The exponential decaying waves, caused by the total internal reflection of incident light, couples with the SPPs on the air side. In (b), a gold film is placed above the prism, which is close enough for the coupling of SPPs with the exponential decaying waves. These two configurations both generate evanescent waves to provide the momentum-matching condition to drive conduction electrons coherently, and therefore generate SPPs on the metal film.

The propagation of SPPs on a metal film can be observed by a leakage radiation microscopy [24] [25] [26]. The principle and schematic of the leakage radiation microscopy will be discussed in Chapter 3 .

\subsubsection{The dispersion relation of SPP}

The electric field of the SPP travelling along the interface between a dielectric film and a metallic film can be expressed as:

$$
\begin{aligned}
& \vec{E}_{d}(x, z, t)=E_{d 0} e^{i\left(k_{x} x-\omega t\right)} e^{-k_{z 1} z} \\
& \vec{E}_{m}(x, z, t)=E_{m 0} e^{i\left(k_{x} x-\omega t\right)} e^{-k_{z 2} z}
\end{aligned}
$$

$\vec{E}_{d}$ in Eq. (2.4) is the electric field in the dielectric film with a real and positive relative permittivity $\epsilon_{1}$, and $\overrightarrow{\mathrm{E}}_{\mathrm{m}}$ in Eq. (2.5) is the electric field in the metal film with a complex relative permittivity $\epsilon_{2}=\epsilon_{2}^{\prime}+i \epsilon_{2}^{\prime \prime}$, where $\epsilon_{2}^{\prime}<0$, $\epsilon_{2}^{\prime \prime}>0 . \omega$ is the angular frequency, $k_{x}$ is the $\mathrm{x}$-axis wave vector along the interface $\mathrm{x}=0, \mathrm{k}_{\mathrm{z} 1}$ is the $\mathrm{z}$-axis wave vector in the dielectric film and $\mathrm{k}_{\mathrm{z} 1}$ is the z-axis wave vector in the metal film. Eqs. (2.4) and (2.5) represents the SPP travelling towards $\mathrm{x}$ direction and exponentially decaying in the $\mathrm{z}$ 
direction. By imposing the appropriate continuity relation of boundary condition $\epsilon_{1} k_{z 2}=\epsilon_{2} k_{z 1}$ by solving Maxwell equations, we get

$$
\begin{array}{ll}
k_{x}=k_{0} \sqrt{\frac{\epsilon_{1} \epsilon_{2}}{\epsilon_{1}+\epsilon_{2}}} & \\
k_{z j}=k_{0} \frac{\epsilon_{j}}{\sqrt{\epsilon_{1}+\epsilon_{2}}} & j=1,2
\end{array}
$$

From Eqs. (2.6), (2.7), we have

$$
\begin{aligned}
& k_{x}=k_{0} \sqrt{\frac{\epsilon_{1}\left[\epsilon_{2}^{\prime}\left(\epsilon_{2}^{\prime}+\epsilon_{1}\right)+\epsilon_{2}^{\prime \prime 2}\right]}{\left(\epsilon_{2}^{\prime}+\epsilon_{1}\right)^{2}+\epsilon_{2}^{\prime \prime 2}}\left[1+i \frac{\epsilon_{1} \epsilon_{2}^{\prime \prime 2}}{\epsilon_{2}^{\prime}\left(\epsilon_{2}^{\prime}+\epsilon_{1}\right)+\epsilon_{2}^{\prime \prime 2}}\right]} \\
& k_{z 1}=k_{0} \sqrt{\frac{\epsilon_{2}^{\prime}\left(\epsilon_{2}^{\prime}+\epsilon_{1}\right)+\epsilon_{2}^{\prime \prime 2}\left(\epsilon_{2}^{\prime}-\epsilon_{1}\right)}{\left(\epsilon_{2}^{\prime}+\epsilon_{1}\right)^{2}+\epsilon_{2}^{\prime \prime 2}}\left\{1+i \frac{\epsilon_{2}^{\prime \prime 2}\left[\epsilon_{2}^{\prime}\left(\epsilon_{2}^{\prime}+2 \epsilon_{1}\right)+\epsilon_{2}^{\prime \prime 2}\right]}{\epsilon_{2}^{\prime \prime}\left(\epsilon_{2}^{\prime}+\epsilon_{1}\right)+\epsilon_{2}^{\prime \prime 2}\left(\epsilon_{2}^{\prime}-\epsilon_{1}\right)}\right\}} \\
& k_{z 2}=k_{0} \sqrt{\frac{\epsilon_{1}^{2}\left(\epsilon_{2}^{\prime}+\epsilon_{1}\right)}{\left(\epsilon_{2}^{\prime}+\epsilon_{1}\right)^{2}+\epsilon_{2}^{\prime \prime 2}}\left(1-i \frac{\epsilon_{2}^{\prime \prime 2}}{\left(\epsilon_{2}^{\prime}+\epsilon_{1}\right)}\right)},
\end{aligned}
$$

where $k_{0}$ is the wave vector of incident light. Eqs. (2.8) - (2.10) can be further simplified by assuming $\epsilon_{2}^{\prime}<0,\left|\epsilon_{2}^{\prime}\right|<\epsilon_{1}$, and $\epsilon_{2}^{\prime \prime} \ll\left|\epsilon_{2}^{\prime}\right|$, we have [17]

$$
\begin{aligned}
& k_{x} \approx k_{0} \sqrt{\frac{\epsilon_{1} \epsilon_{2}^{\prime}}{\epsilon_{2}^{\prime}+\epsilon_{1}}}\left[1+i \frac{\epsilon_{1} \epsilon_{2}^{\prime \prime}}{2 \epsilon_{2}^{\prime}\left(\epsilon_{2}^{\prime}+\epsilon_{1}\right)}\right] \\
& k_{z 1} \approx k_{0} \frac{\epsilon_{2}^{\prime}}{\sqrt{\epsilon_{2}^{\prime}+\epsilon_{1}}}\left(1+i \frac{\epsilon_{2}^{\prime 2}}{2 \epsilon_{2}^{\prime}}\right) \\
& k_{z 2} \approx k_{0} \frac{\epsilon_{1}}{\sqrt{\epsilon_{2}^{\prime}+\epsilon_{1}}}\left(1-i \frac{\epsilon_{2}^{\prime \prime 2}}{2\left(\epsilon_{2}^{\prime}+\epsilon_{1}\right)}\right)
\end{aligned}
$$

However, in Ref. [27] it was shown that, comparing with Eqs. (2.8) - (2.10), Eqs. (2.11) - (2.13) give inaccurate results for the case study of $\mathrm{Ag} / \mathrm{GaP}$ even under assumptions of $\epsilon_{2}^{\prime}<0,\left|\epsilon_{2}^{\prime}\right|<\epsilon_{1}$, and $\epsilon_{2}^{\prime \prime} \ll\left|\epsilon_{2}^{\prime}\right|$. 
(a)

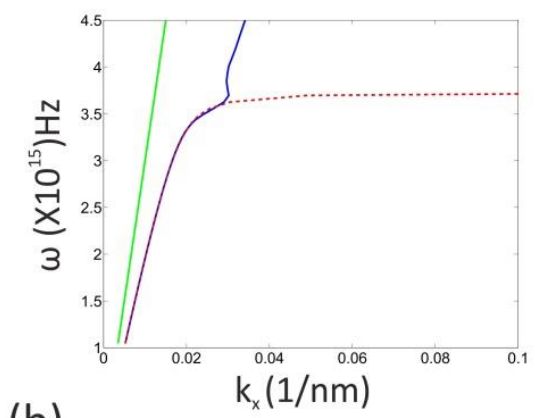

(b)

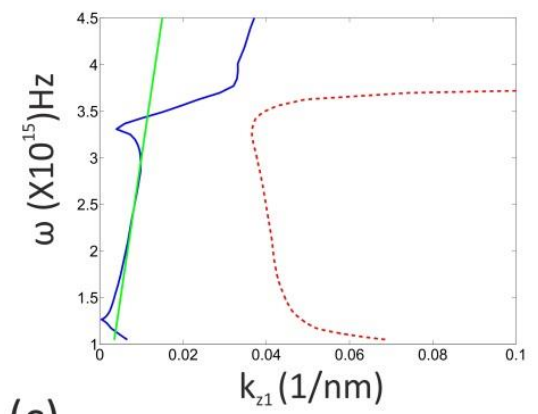

(c)

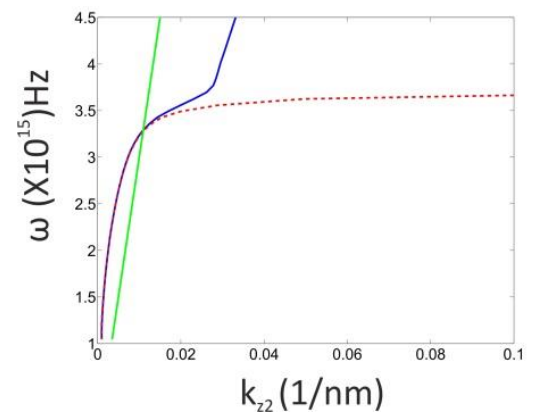

Fig. 2.3: The dispersion relation of gold on glass: (a) $\omega\left(k_{x}\right)$; (b) $\omega\left(k_{z 1}\right)$; and (c) $\omega\left(k_{z 2}\right)$. Blue curves are calculated by Eqs. (2.8) - (2.10) and red dashed curves are calculated by Eqs. (2.11) (2.13). Green curves are the light line in air. 


\section{Chapter 2}

Following the protocol introduced in [27] we calculate the dispersion relation for the case of $\mathrm{Au} / \mathrm{SiO}_{2}$ by Eqs. (2.8) - (2.10) and (2.11) - (2.13). The dielectric constants of $\mathrm{Au}$ and $\mathrm{SiO}_{2}$ are taken from experimental results published in Refs. [22] and [28], respectively.

Fig. 2.3 shows results of the calculated dispersion relations of gold on glass: $\omega\left(k_{x}\right), \omega\left(k_{z l}\right)$ and $\omega\left(k_{z 2}\right)$. The blue curves in Fig. 2.3(a) - (c) are calculated by Eqs. (2.8) - (2.10); red dashed curves are calculated by Eqs. (2.11) - (2.13) and green curves are light line in air. In (b), the blue curve has totally different values than the red dashed curve, while in (a) and (c), the blue curve differs as $\omega$ is above $3.6 \times 10^{15} \mathrm{~Hz}$.

We can conclude that Eqs. (2.11) - (2.13), appearing in most plasmonics textbooks, fails in describing the dispersion relation of $k_{z l}$ (the z-axis wave vector in the $\mathrm{SiO}_{2}$ layer), and should be used with caution of the wavelength region on the dispersion relation of $k_{x}$ and $k_{z 2}$ (the z-axis wave vector in the gold layer).

\subsubsection{Localized surface plasmon (LSP)}

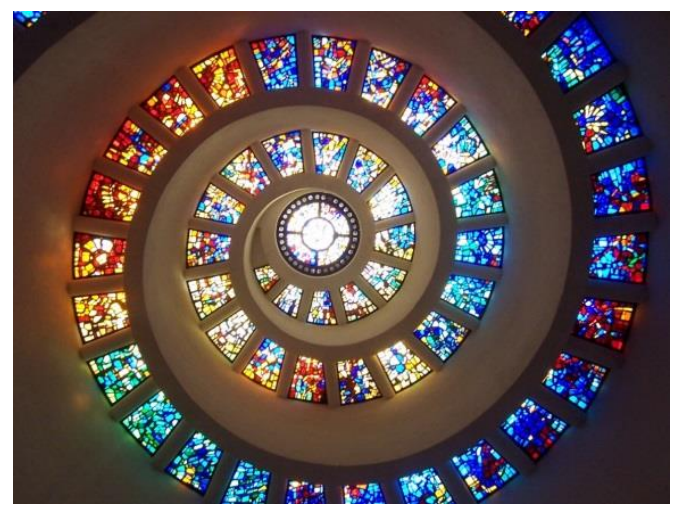

Fig. 2.4: A view of the stained glass ceiling at Thanksgiving square in Dallas Texas.

In addition to metal thin films, surface plasmons can be generated in a small metal nanoparticles, too. The conduction electrons in a metal nanoparticle will oscillate coherently when incident light is at the resonant wavelength of 
the plasmon oscillation in the metal nanoparticle. The resonant wavelength of a metal nanoparticle depends on its size, shape and the dielectric function of the metal nanoparticle.

Fig. 2.4 shows a beautiful example of the different resonance wavelengths of LSPs associated with various materials. The stained glass, a popular decoration of windows in churches for thousands of year, contains small metal particles with different LSP resonant wavelengths, which renders different colors as sunlight shines through. Note that, at the resonant wavelength of LSPs, light is absorbed. The color of glass doesn't correspond to the resonance wavelength.

\subsubsection{Localized surface plasmon resonance (LSPR)}

The electric field of a LSP in a metal nanoparticle can be calculated by considering a light incident on a spherical metal particle of radius $a$ in the electrostatic limit, which is equivalent to ignoring the spatial variation of the incident electric field inside the particle. In the electrostatic picture, an electric field only induces charges on the surface of the particle. Hence, the total electrostatic potential is the sum of an incident light part and the contribution generated by the surface charges. With appropriate boundary conditions providing continuity of the tangential part of electric fields $E$ and the longitudinal part of the electric displacements $D$ on the surface of the metal nanoparticle, the electric field can be solved. The electric field inside the metal nanoparticle is constant: $E_{0} \frac{3 \epsilon_{2}}{\epsilon_{1}+2 \epsilon_{2}} \hat{e}_{x}$, where $\epsilon_{1}$ is the dielectric constant of the metal nanoparticle, $\epsilon_{2}$ is the dielectric constant of the medium outside the metal nanoparticle, and $\hat{e}_{x}$ is the polarization of the incident light. The electric field outside the metal nanoparticle is [23]:

$$
E_{\text {out }}=E_{0}\left(\cos \theta \hat{e}_{r}-\sin \theta \hat{e}_{\theta}\right)+\frac{\epsilon_{1}-\epsilon_{2}}{\epsilon_{1}+2 \epsilon_{2}} \frac{a^{3}}{r^{3}} E_{0}\left(2 \cos \theta \hat{e}_{r}+\sin \theta \hat{e}_{\theta}\right)
$$

The first term in Eq. (2.14) is the electric field of incident light. The second term is the electric field of the scattered light by the metal nanoparticle, which can be regarded as the electric field of a dipole $\mu$ located at the center of the sphere. The dipole has the value $\mu=\epsilon_{2} \alpha(\omega) E_{0}$, where $\alpha(\omega)$ is the polarizability 


$$
\alpha(\omega)=4 \pi \epsilon_{0} a^{3} \frac{\epsilon_{1}(\omega)-\epsilon_{2}}{\epsilon_{1}(\omega)+2 \epsilon_{2}} .
$$

From the denominator of Eq. (2.15), the polarizability $\alpha$ diverges as $\epsilon_{1}(\omega)=$ $-2 \operatorname{Re}\left(\epsilon_{2}\right)$. This is the resonance condition for a collection for the collective electron oscillation in the metal nanoparticle. For a metal nanorod, the resonant condition becomes $\epsilon_{1}(\omega)=-\operatorname{Re}\left(\epsilon_{2}\right)$.

This resonant condition of the LSPR gives us the information of the excitation of LSPR of metal nanoparticles in fractal optical antennas in subsequent chapters. The LSPR of a metal nanoparticle in fractal optical antennas can be modelled as a dipole with the dipole moment given by Eq. (2.15). Details will be discussed in Chapter 4 and 6.

\subsection{Introduction of optical antennas}

(a)

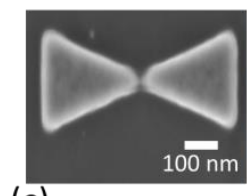

(c) (b)
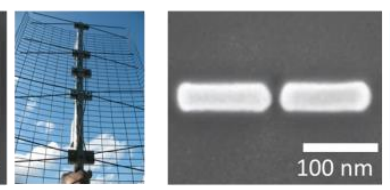

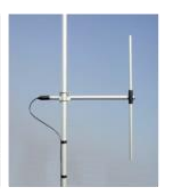

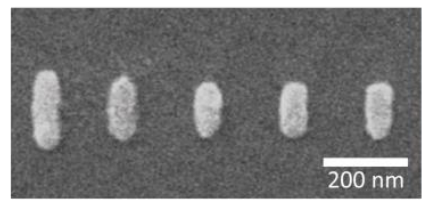

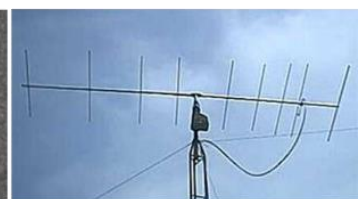

Fig. 2.5: Examples of SEM images of the optical antennas and photos of their counterpart in radio frequency regimes. (a) left: optical bowtie antenna, right: bowtie antenna (image adapted from SuperQuad 4 Bay Bowtie from Kosmic Antennas). (b) left: optical dipole antenna, right: dipole antenna (image adapted from Sirio WD140-N VHF 140-160 MHz Base Station). (c) left: optical Yagi-Uda antenna, right: Yagi-Uda antenna (Optimized 6/9 element VHF Yagi Antenna, RE-A144Y6/9). SEM photos are adapted from [18].

Optical antennas are the counterpart of radio or microwave antennas in optical frequency regimes. Radio or microwave antennas are widely used to 
convert propagating electromagnetic waves into localized energy and vice versa. In the optical frequency regime, however, analogous localizers for optical radiation, i.e. optical antennas, haven't been implemented until recently [18] [19]. This is because the characteristic dimensions of antennas are in the order of the radiation wavelength, which for the optical regime requires fabrication accuracies down to a few nanometers. This length scale has only recently becomes accessible due to the progress of fabrication tools. The top-down fabrication tools such as focused ion beam milling or electronbeam lithography, and the bottom-up self-assembly schemes facilitate the fabrications of optical antennas with different designs.

Fig. 2.5 shows optical bowtie (a) and dipole (b) antennas fabricated by focused ion beam milling; (c) shows an optical Yagi-Uda antenna fabricated by electron-beam lithography. Their counterparts in the radio-frequency regimes are also shown for comparison. In Chapter 3, we will introduce the details of the fabrication of optical antennas used in this thesis. This section will focus on the their characteristic properties.

\subsubsection{The bright and dark plasmonic mode}

For a single metal nanoparticle, the electromagnetic mode is rather simple: its dipole moment just follows the polarization of the incident radiation with a phase delay. The radiation pattern of a single metal nanoparticle can be regarded as that of a dipole. The radiation damping causes the broadening of the spectral width of the EM mode.

For an optical antenna composed of two metal nanoparticles, the electromagnetic modes can be of two types: dark and bright modes. The dark mode is illustrated in Fig. 2.6(a). Here we use color to represent the polarization direction of the dipole moment of metal nanoparticles. The blue and red colors in (a) indicate any equal quantity but opposite polarization directions of dipole moment of two metal nanoparticles. Similarly, in Fig. 2.6(b), the red color of two metal nanoparticles represents their dipole moment pointing at the same direction.

Intuitively, one can understand that the dark mode in Fig. 2.6(a) can not be excited directly by a plane wave, but the bright mode in Fig. 2.6(b) can be directly excited. This statement can be extended to understand a key property of the dark sand bright mode: a dark mode couples weakly with an incident 
polarized light from far field, while a bright mode will couple strongly. Furthermore, the cancellation of dipole moments in dark modes of the optical antenna inhibits its radiation to be transferred to the far field and consequently reduce the radiation loss, which renders the light energy stored in the near field of the optical antenna. Therefore, the dark mode can store energy more efficiently and has higher near-field intensity enhancement than the bright mode.

In the literature, the dark and bright modes in optical antennas are sometimes also called "subradiant" and "superradiant" modes, respectively [29, 30, 31, 32, 33, 34]. The minor difference between "dark" and "subradiant" can sometimes be confusing. In an ideal plasmonic system, the dark mode can be defined rigorously as a mode with a zero net dipole moment, while subradiant mode has a relatively low but nonzero dipole moment comparing with other bright or superradiant modes of optical antennas. In reality, a plasmonic system can never have a vanishing net dipole moment because of the imperfection of sample fabrications. Therefore, in this thesis, we regard "dark mode" as having the same meaning as "subradiant mode", and "bright mode" as having the same meaning as "superradiant mode".

(a)

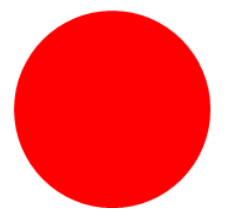

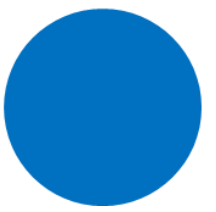

(b)
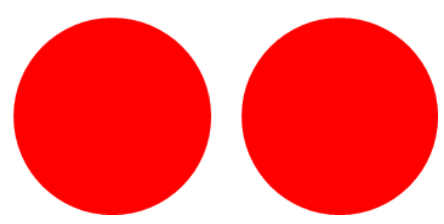

Fig. 2.6: (a) the dark and (b) the bright plasmonic mode an optical antenna composed of two metal nanoparticles. The blue and red color represent the opposite polarization directions of dipole moments of metal nanoparticles in (a), while red color in (b) shows that the polarization direction of both metal nanoparticles are in the same direction. 


\subsubsection{The Fano resonance in optical antennas}

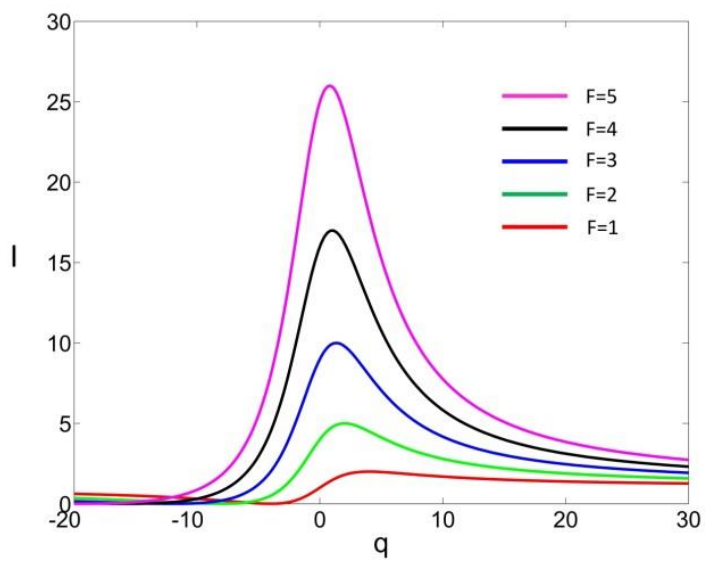

Fig. 2.7: The profiles of Fano resonances for F from 1 to 5.

Due to the reduced radiation damping of dark modes as mentioned above, methods of excitation and observation of dark modes in optical antennas have drawn a lot of attention. The zero net dipole moment renders a dark mode which can not be directly excited by a linear polarized light from far field. However, a dark mode can be excited indirectly through a coupling with a bright mode in optical antennas. This coupling between dark and bright modes gives rise to a feature called the "Fano resonance" [35] in extinction or scattering spectra of optical antennas. The interference between a bright mode and a dark mode is similar to the case of the interference between a continuum state (a background process) and a discrete state (a resonant process). The spectral feature of Fano resonance can be expressed as [36]:

$$
I \approx \frac{\left(F \gamma+\omega-\omega_{0}\right)^{2}}{\left(\omega-\omega_{0}\right)^{2}+\gamma^{2}}
$$

where $\omega_{0}$ and $\gamma$ are the spectral position and width of the resonance, respectively. $F$ is the so-called Fano parameter. Eq. (2.16) can be simplified to 


$$
I \approx \frac{(F+q)^{2}}{q^{2}+1}
$$

where $q=\frac{\omega-\omega_{0}}{\gamma}$.

Fig. 2.7 shows the profiles of Fano resonance from Eq. (2.17) for different values of $F$ from 1 to 5 . The constructive and destructive interference between the continuum state and the discrete state changes the Lorentzian resonance into an asymmetric profile.

The interference between a dark and a bright mode in an optical antenna can be understood to be a Fano-like resonance. The relatively broader spectral response of a bright mode is regarded as the continuum state. For a dark mode which has narrower spectral response due to the reduced radiation damping, it can be regarded as a discrete state. This interference between a dark and a bright mode can be modelled by equations of a coupled oscillator [37]:

$$
\begin{aligned}
& \ddot{p}(t)+\gamma_{b} \dot{p}(t)+\omega_{b}^{2} p(t)+\kappa q(t)=g E(t) \\
& \ddot{q}(t)+\gamma_{d} \dot{q}(t)+\omega_{d}^{2} q(t)+\kappa p(t)=0
\end{aligned}
$$

$p(t)$ and $q(t)$ are coordinate of the bright and dark mode, respectively. Eq. (2.18) describes the equation of motion of the bright mode, which has a damping factor $\gamma_{b}$ and resonance frequency $\omega_{b}$. The $g E(t)$ is the external force for the bright mode. Eq. (2.19) describes the equation of motion of the dark mode, which has a damping factor $\gamma_{d}$ and resonance frequency $\omega_{d}$. Two oscillators are coupled with a coupling strength $\kappa$. Eqs. (2.18), (2.19) can be solved in the frequency domain by assuming the form $p(t)=\tilde{p}(\omega) e^{-i \omega t}$ and $q(t)=\tilde{q}(\omega) e^{-i \omega t}$ :

$$
\begin{aligned}
& \tilde{p}(\omega)=\frac{D_{d}(\omega) g \tilde{E}(\omega)}{D_{d}(\omega) D_{b}(\omega)-\kappa^{2}} \\
& \tilde{q}(\omega)=\frac{\kappa g \tilde{E}(\omega)}{D_{d}(\omega) D_{b}(\omega)-\kappa^{2}},
\end{aligned}
$$


(a)

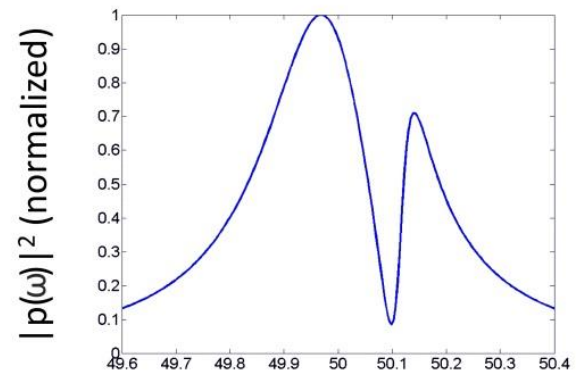

(b)

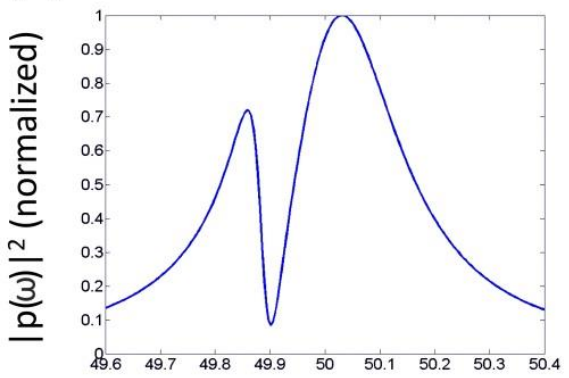

(c)

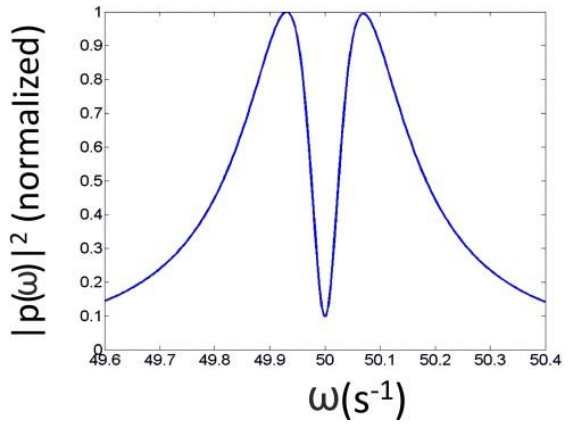

Fig. 2.8: $|\tilde{p}(\omega)|^{2}$ profiles with (a) $\omega_{d}=\omega_{b}+0.1 s^{-1}$ (b) $\omega_{d}=\omega_{b}-0.1$ (c) $\omega_{d}=\omega_{b}$. 
where $D_{b}(\omega)=1-\left(\frac{\omega}{\omega_{b}}\right)^{2}-i \gamma_{b}\left(\frac{\omega}{\omega_{b}^{2}}\right), D_{d}(\omega)=1-\left(\frac{\omega}{\omega_{d}}\right)^{2}-i \gamma_{d}\left(\frac{\omega}{\omega_{d}^{2}}\right)$ and $E(t)=\tilde{E}(\omega) e^{-i \omega t}$. In the interference of a bright and a dark mode, the damping factor for the bright mode $\gamma_{b}$ is much larger than that of the dark mode: $\gamma_{b} \gg \gamma_{d}$. Eqs. (2.20) - (2.21) can be also used as a classical model for the phenomenon of electromagnetically induced transparency (EIT) [38].

Fig. 2.8 shows the profiles of $|\tilde{p}(\omega)|^{2}$ with different $\omega_{d}$ as $\omega_{b}=50 \mathrm{~s}^{-1}$, $\kappa=6 \mathrm{~s}^{-2}, \gamma_{b}=0.3 \mathrm{~s}^{-1}$ and $\gamma_{d}=0.02 \mathrm{~s}^{-1}$. The dip in Fig. 2.8(a) - (c) comes from the destructive interference between the bright and dark modes. For an optical antenna, the dip in the extinction or scattering spectrum caused by the destructive interference of a dark mode and a bright mode is called the "Fano-dip". This is illustrated further in Fig. 2.9, adapted from Ref. [39]. Fig. 2.9(a) shows the extinction spectrum of a gold heptamer. The Fano-dip (marked D) comes from the destructive interference between a bright mode shown in Fig. 2.9(b) and a dark mode shown in Fig. 2.9(c). The simulated electric current in (b) and (c) are represented by blue arrows. The central gold nanoparticle has an opposite direction of electric current with respect to that of other gold nanoparticles, which reduces the net dipole moment of the dark mode. The simulated near-field intensity are presented by the color scale. It is obvious to see that the near-field intensity of dark mode shown in (c) is higher than that of the bright mode shown in (b).

(a)

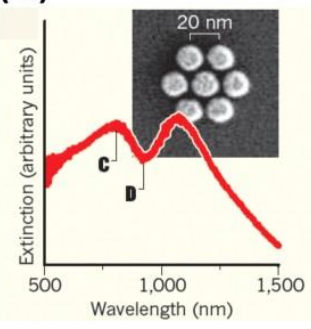

(b)

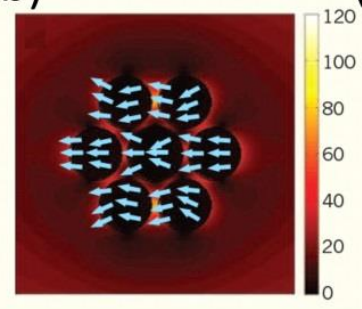

(c)

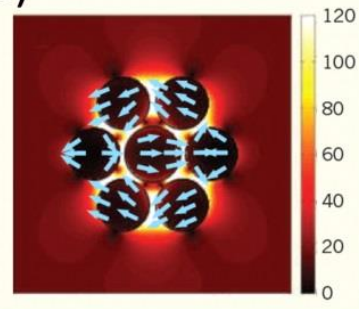

Fig. 2.9: (a) The extinction spectrum of a gold heptamer. The inset is the SEM picture of the heptamer. (b), (c) The simulated near-field intensity and corresponding electric currents (blue arrows) at the spectral wavelengths marked C and D in (a). Figures are adapted from [39]. 


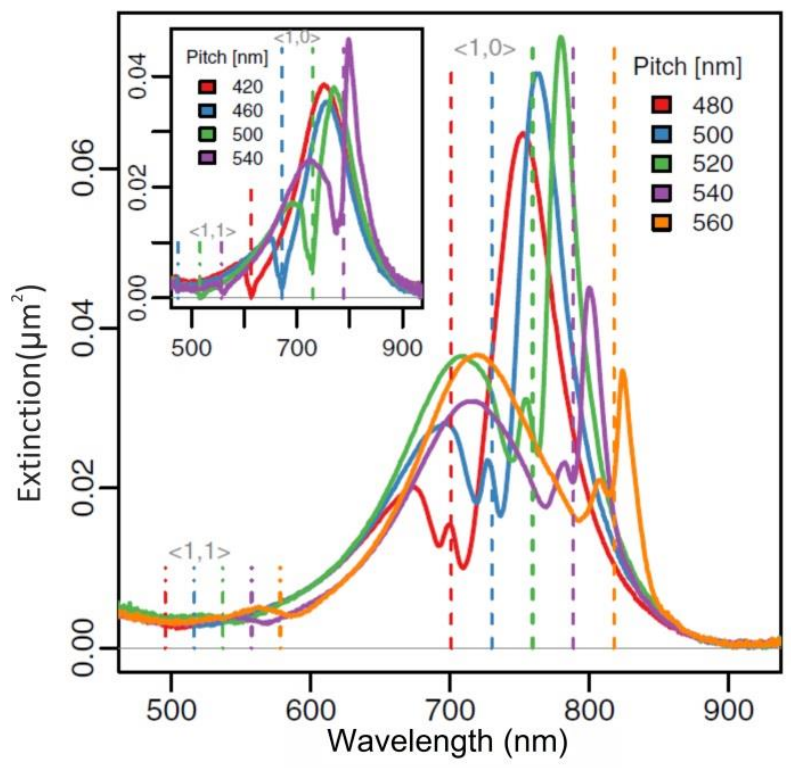

Fig. 2.10: Measured extinction spectra for several gold nanoparticle periodic arrays. The particle size is $123 \mathrm{~nm} \times 85 \mathrm{~nm} \times 35 \mathrm{~nm}$. Inset (particle size $120 \mathrm{~nm} \times 90 \mathrm{~nm} \times 35 \mathrm{~nm}$ ): When the diffraction edge is on the blue side of the main resonance, a much weaker effect is observed. $\langle 1,0\rangle$ and $<1,1>$ are diffraction direction for a periodic array. The figure is adapted from [40].

Besides the heptamer, the extinction spectrum of a periodic array of metal particles also exhibit another type of Fano resonance-the surface lattice resonance (SLR) [41, 42, 43, 44, 40, 45]. A SLR is a specific collective mode of metal particles in a periodic array, which comes from the intricate coupling of LSPR and in-plane diffracted waves. The SLR generates a sharp spectral feature or a Fano-dip depending on the relative spectral position of LSPR of monomers with respect to spectral position where inplane diffracted waves occur (or called "the Rayleigh condition") [40]. Therefore, the SLR strongly depends on the size and shape of metal particles (determining the spectral position of LSPR), and the pitch size of a periodic metal particle arrays (determining the spectral position of in-plane diffracted waves). Fig. 2.10 shows experimental spectra of a SLR in the extinction spectra of periodic arrays of gold particles; adapted from Ref.[40]. We can 


\section{Chapter 2}

find that sharp features of the SLR appear in extinction spectra when the inplane diffracted waves occur on the red side (the lower photon energy) of the spectral position of the LSPR of a single gold particle, while a Fano-dip appears when the in-plane diffracted waves occur on the blue side (the higher photon energy) of the spectral position of the LSPR of a single gold particle.

In Chapter 6, we will discuss a SLR in a Sierpinski carpet optical antenna. Different from the SLR in a periodic metal particle array measured by the extinction spectra, we will visually show the SLR of the Sierpinski carpet optical antenna by a back focal plane microscopy. 


\section{Chapter 3}

\section{Sample fabrication and characterization methodologies}

In contrast to the multi-crystalline gold films typically used as starting material for the fabrication of optical antennas, here chemically-synthesized single-crystalline gold flakes are employed. The recipe and procedure for synthesizing single-crystalline gold flakes is introduced, as well as the method of directly carving samples using focused ion beam (FIB) milling on gold flakes. The results of fabricating gold nanostructures on both a thermal evaporated multi-crystalline gold film and on a single-crystalline gold flake are compared and discussed. The working principles and importance of the various instrumentations used in the characterization of samples are presented in the end of this chapter, including white light dark-field microscopy, two-photon photoluminescence microscopy and back focal plane microscopy. 


\subsection{Fabrication of optical antennas}

The performance of plasmonic nanostructures is greatly influenced by the quality of metal materials. Multi-crystalline metal thin films (below $100 \mathrm{~nm}$ ) produced by thermal evaporation have been widely used in the fabrication of plasmonic nanostructures. However, irregular granular boundaries are formed in a metal thin film during the thermal evaporation process of metal onto a glass substrate. These random-oriented grains (usually $30-50 \mathrm{~nm}$ diameter) have different resistance to FIB milling, which causes unwanted features with comparable grain sizes after the fabrication of plasmonic nanostructures. These features introduce scattering, which reduces the lifetime of plasmons.

Recently, single crystalline gold flakes have been chemically synthesized and applied for the fabrication of plasmonic nanostructures with well-defined shapes [46]. In this section the synthesis of single-crystalline gold flakes and the fabrication of fractal optical antennas on these flakes by FIB milling are introduced.

\subsubsection{Synthesis of single-crystalline gold flakes}

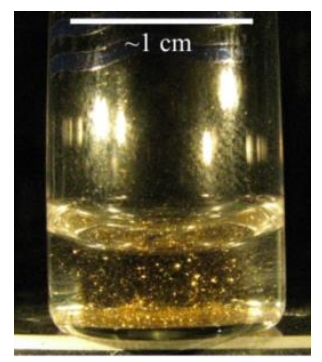

Fig. 3.1: Single crystalline gold flakes and particles, dispersed in water solution.

The specific steps of synthesizing single-crystalline gold flakes follow the procedure described in Ref. [47]. It begins with hydrogen tetrachloroaurate (HAuCL4), dissolved in ethylene glycol. Poly (vinyl pyrrolidone) (PVP) is then added as capping agent. The mixed solution is heated in an oil bath to approximately $150{ }^{\circ} \mathrm{C}$ and after two hours the gold flakes appear in the solution as shown in Fig. 3.1. 


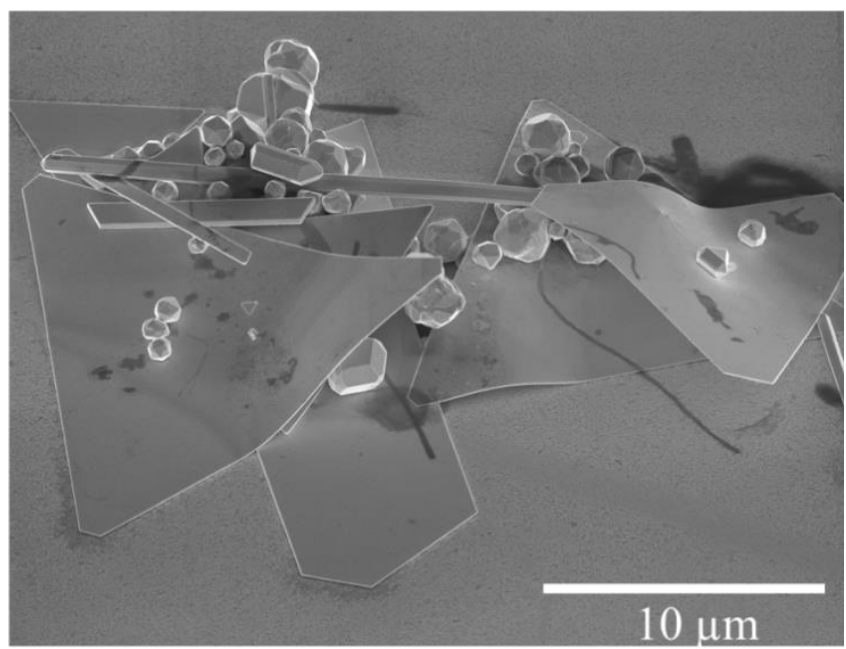

Fig. 3.2: The chemically-synthesized single crystalline gold flakes with some gold nanoparticles and nanowires on top of them, which are also formed during the synthesis process.

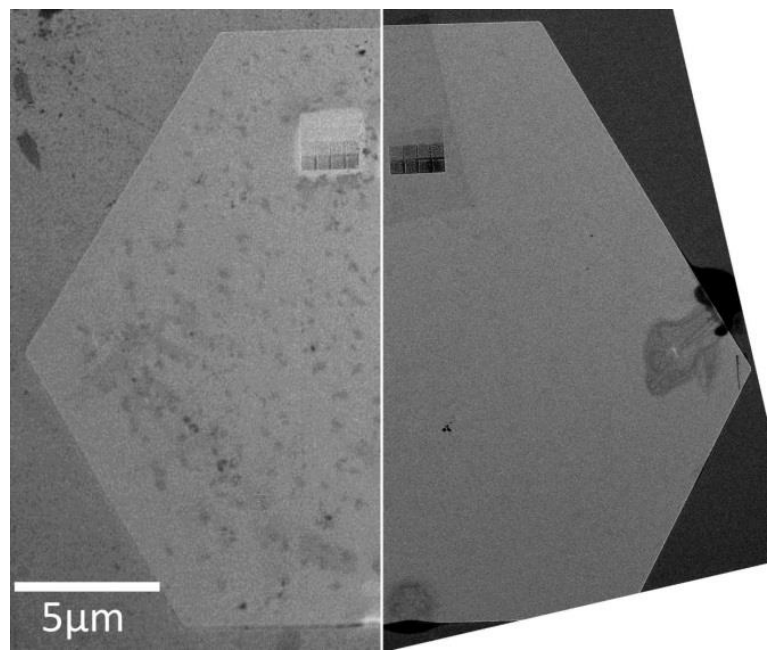

Fig. 3.3: A single-crystalline gold flake before (left) and after (right) the $\mathrm{O}_{2}$ plasma cleaning. Most of contaminations on the surface of flake are removed after cleaning. 


\section{Chapter 3}

Samples can be prepared by dropcasting the gold flakes and water mixture onto the glass substrate and subsequently removing water drops by clean and pressured nitrogen gas. Fig. 3.2 shows some gold flakes, gold nanowires and nanoparticles on a glass substrate with a $100 \mathrm{~nm}$ thickness conductive ITO layer. Also note that there are a few dark spots on gold flakes, which is due to solvent residues from the manufacturing process.

\subsubsection{Removing residual chemicals on a gold flake by $\mathrm{O}_{2}$ plasma cleaning}

The oxygen plasma cleaning process can be used to remove residual contaminants (probably organic) remaining on a gold flake after the chemical synthesis process. This cleaning step takes about 10 seconds. Fig. 3.3 shows that most of contaminants have been removed after the $\mathrm{O}_{2}$ plasma cleaning. This creates a uniform and smooth surface of the gold flake, which facilitates the fabrication of our optical antennas.

\subsubsection{Instrumentation}

(a)

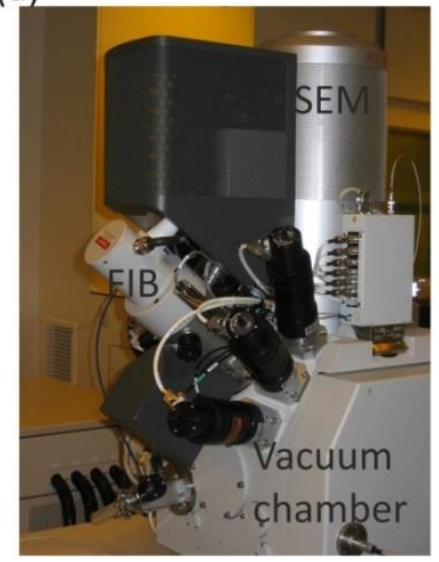

(b)

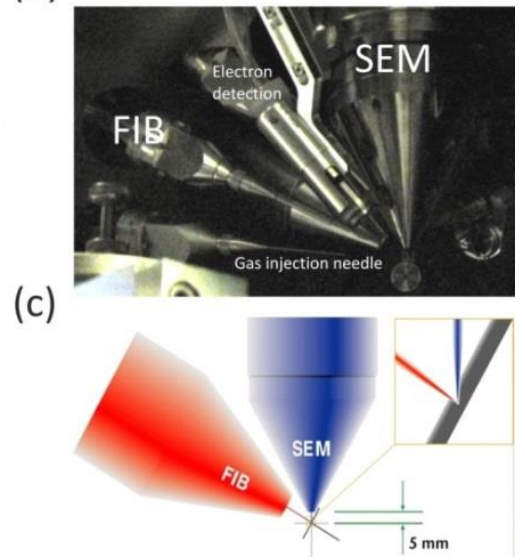

Fig. 3.4: (a) Photos of the FIB instrument. (b) View inside the vacuum chamber. (c) Schematic representation of the dual-beam configuration. 
To fabricate our samples, we used focused ion beam (FIB) milling (FEI Nova 600 dual beam) to carve nanostructures directly on single-crystalline gold flakes. In the Fig. 3.4(a), two columns in the machine can be identified. The FIB column is angled at 52 degrees relative to the vertical scanning electron microscopy column. The sample stage can be moved with 5 degrees of freedom for SEM imaging or FIB milling. Fig. 3.5 shows schematics of the FIB and scanning electron microscopy columns. The Ga LMIS (liquid-metal ion sources) consists of a tungsten needle with a heater to supply Ga to the tip. $\mathrm{Ga}$ remains liquid after heating once. $\mathrm{Ga}$ is chosen for its durability (1500 hours) and stability (heating every 100 hours).

FIB column

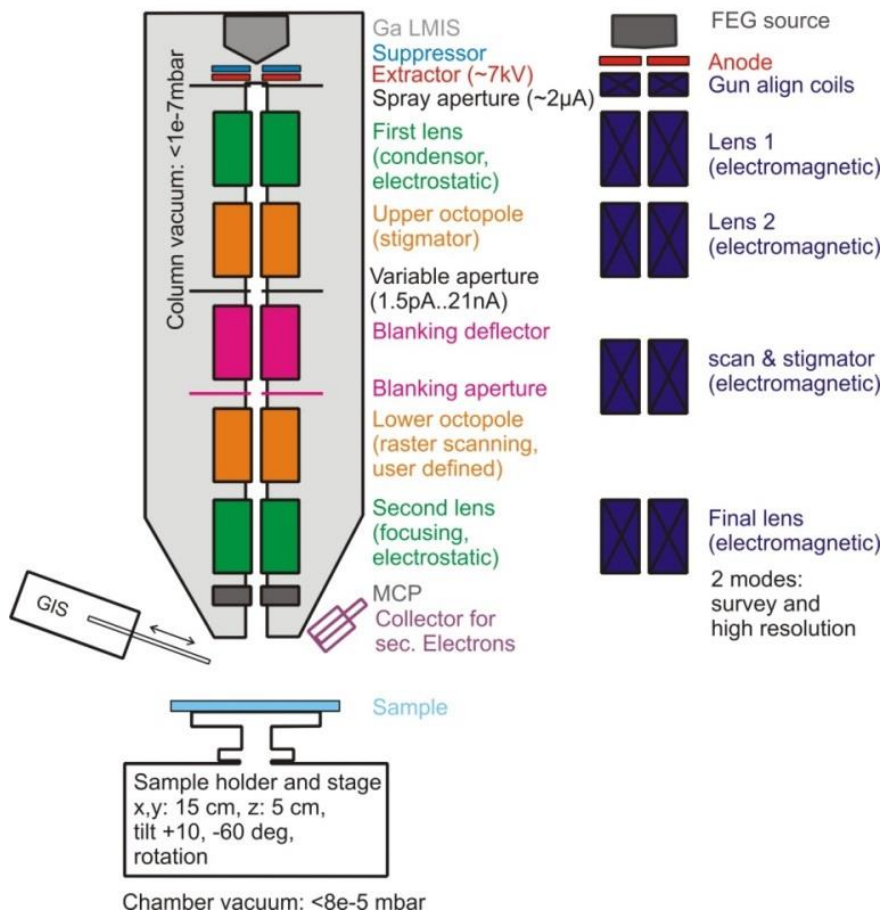

SEM column

Fig. 3.5: The schematics of a FIB (left) and scanning electron microscopy column (right). LMIS: liquid-metal ion source ; FEG: Field emission Gun. The image is adapted from [48]. 


\subsubsection{Fabrication result of the multi- crystalline gold film and single-crystalline gold flakes}

The advantages of using single-crystalline gold flakes for antenna fabrication can be readily seen in Fig. 3.6. A single-crystalline gold flake (about $100 \mathrm{~nm}$ thickness) is deposited above a thermal evaporated multi-crystalline gold film (also about $100 \mathrm{~nm}$ thickness) as shown in the schematic on the lower right of Fig. 3.6. The smooth and uniform surface of the single-crystalline gold flake, compared to the multi-crystalline gold film, can be seen immediately. We removed the gold layers by FIB milling to form a 5-by-5 $\mu \mathrm{m}$ square hole. The residue of single-crystalline gold flake is a very thin film. However, for the multi-crystalline gold film, it ends in blobs of gold, which are the result of irregular crystal grains in it. The roughness of residuals of the multicrystalline gold is much higher than that of the single-crystalline gold flake. This shows the superiority of single-crystalline gold flakes for the fabrication of plasmonic nanostructures.

Fig. 3.7(a) shows the fabrication with single-pass milling of a gold cross bar antenna on a multi-crystalline gold film. Panel (b) shows the result of single-pass milling on a single-crystalline gold flake, while (c) shows the fabrication result on a single-crystalline gold flake with multi-pass milling (15 passes) and software drift correction applied. The samples shown in (a) and (b) were both fabricated with single-pass FIB milling; in both residual gold nanoparticles can be seen. The difference is that the residual particle size for the single-crystalline gold flake is much smaller than that of the multi-crystalline gold film. This is because of the larger and irregular crystal grains in the multi-crystalline gold film. We notice that, in (a) and (b), the horizontal bars are wider than vertical ones. This is due to the redeposition of gold particles. During FIB milling process, the ion beam raster-scanned from upper-left to lower-right. This causes the redeposition of removed gold nanoparticles near the lower edges of the horizontal bars. In (c) we can see that the shape of cross bar antenna is well-defined and the background is clean. Therefore, we chose the single-crystalline gold flake and multi-pass FIB milling in combination of position feedback to fabricate our optical antennas. 


\section{Sample fabrication and characterization methodologies}

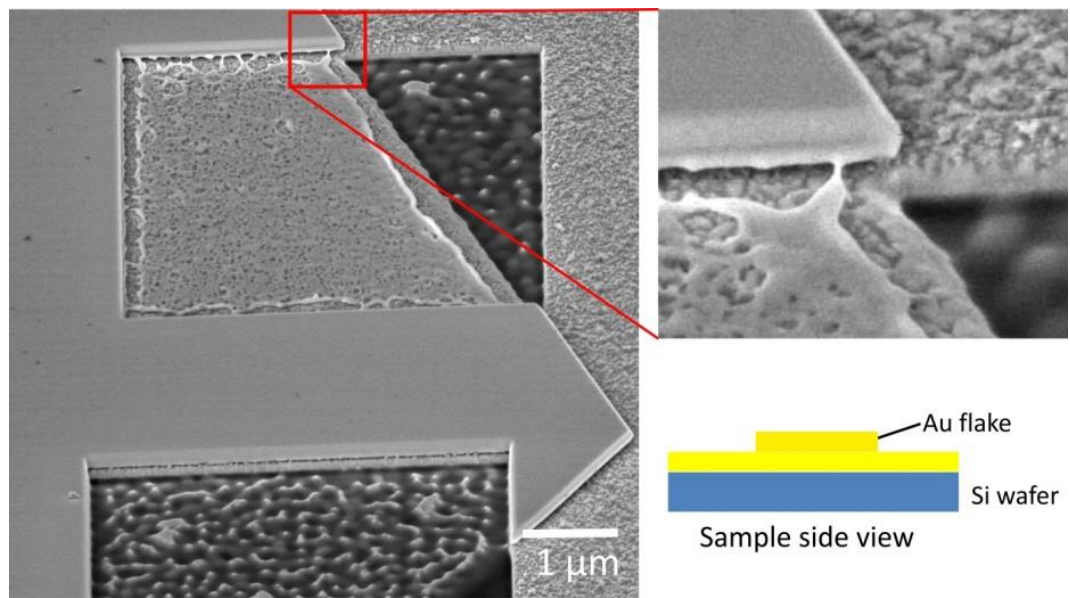

Fig. 3.6: The fabrication result of a single-crystalline gold flake on the top of a multi-crystalline gold thin film.

(a)

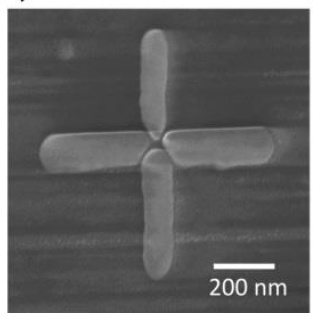

(b)

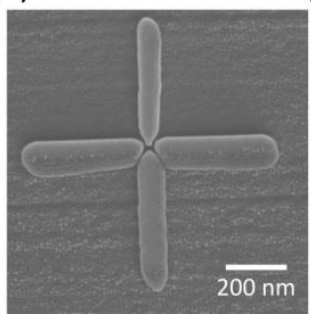

(c)

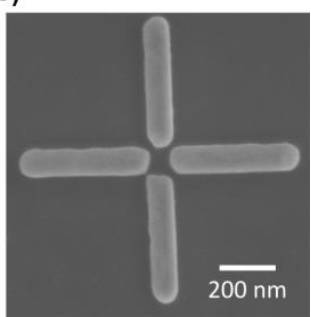

Fig. 3.7: (a) A cross bar nanoantenna fabricated by single-pass FIB milling on a multicrystalline gold film with thickness of $40 \mathrm{~nm}$. Panels (b) and (c) show cross bar nanoantennas fabricated on a single-crystalline gold flake with single-pass and multi-pass (15 passes) milling, respectively. 


\section{Chapter 3}
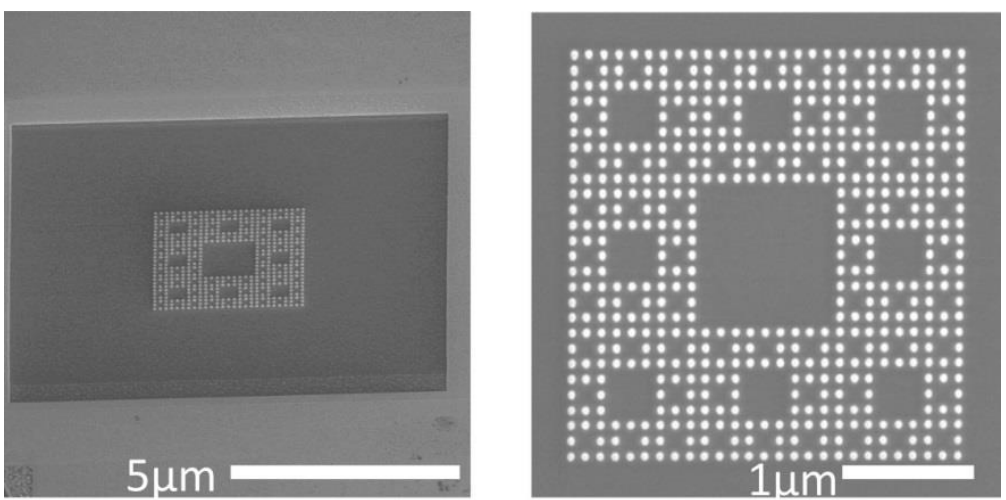

Fig. 3.8: A Sierpinski carpet optical antenna fabricated on a single-crystalline gold flake by FIB milling.

Fig. 3.8 shows an example of the fabrication of a Sierpinski carpet optical antenna on a single-crystalline gold flake by FIB milling (30 pass). The flake was pre-thinned to a thickness of $\sim 40 \mathrm{~nm}$. In FIB milling process, the acceleration voltage was $30 \mathrm{kV}$ and the Ga-ion current was $1.5 \mathrm{pA}$. The monomer size is $80 \pm 8 \mathrm{~nm}$ (diameter) and $40 \pm 5 \mathrm{~nm}$ (thickness); the gap distance between monomers is $30 \pm 11 \mathrm{~nm}$. We can see that the shape of monomers is uniform and the background is clean without residuals of gold nanoparticles. We will discuss the Sierpinski carpet optical antenna in detail in following chapters.

\subsection{Instrumentation}

In this section, I will introduce the instrumentations and techniques used in experimental works of this thesis: the white light dark-field microscopy used for the determination of the resonant wavelength, the two photon photoluminescence for the measurement of the near-field intensity and the back focal plane microscopy for the visualization and quantification of the intensity of plasmonic modes of optical antennas. These measurements are critical in the design and application of optical antennas. 


\subsubsection{White light dark-field microscopy}

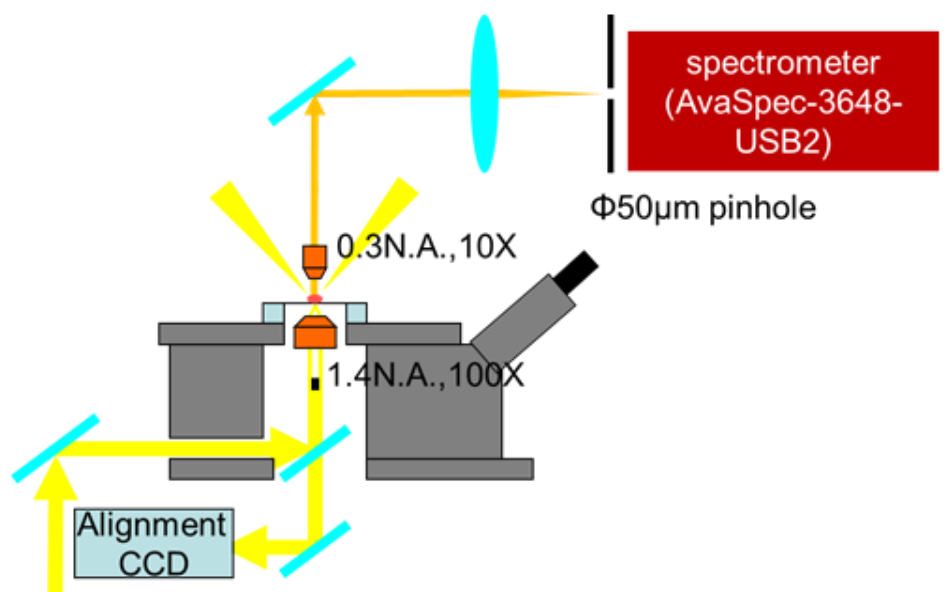

Fig. 3.9: The schematic of white light dark field microscopy. Note the inclusion of a beam block for annular illumination.

In optical microscopy, dark-field describes an illumination technique used to enhance the contrast in unstained samples. It works by illuminating the sample with light that will not be collected by the objective lens, and thus will not form part of the image. This produces the classic appearance of a dark background with bright objects on it [49].

The white light dark-field microscope, utilizing the dark-field illumination configuration, has become a widely-utilized tool in plasmonics research area to determine the resonant wavelength of gold nanostructures [50] [51] [52] [53]. In this technique, an incident beam illuminates the sample of interest, and scattered light from the metal nanostructure is collected by an objective and sent into a spectrometer. The stray light from the substrate is not collected by the objective to minimize background noise. A pinhole before the spectrometer entrance can be used in a confocal like design to ensure that scattered light from only a single nanostructure enters the spectrometer. 


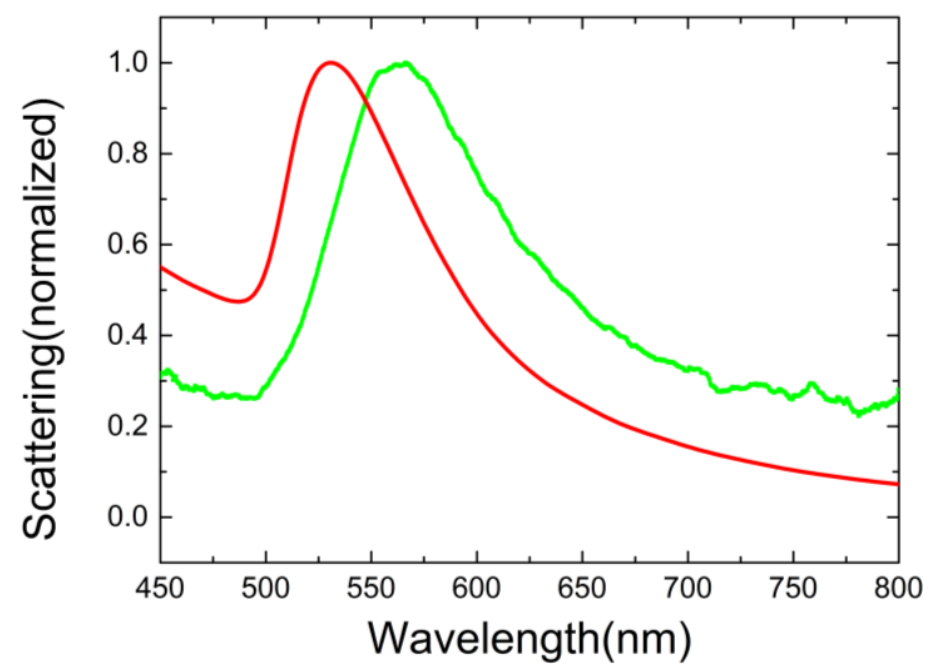

Fig. 3.10: The scattering spectrum of a gold nanoparticle with $100 \mathrm{~nm}$ diameter. The green curve is the experimental data measured by the white light dark-field microscope shown in Fig. 3.9. The red curve is the finite-difference time-domain (CST MWS 2013) simulation result. The more broad and red shift of the experimental result compared with the simulation result come from the glass substrate which is not considered in the simulation.

Our home-built dark-field spectrometer setup [1] is shown schematically in Fig. 3.9. White light from a Xenon Arc lamp (Oriel 71213, Newport) is sent into a microscope (IX71, Olympus) and focused onto samples with a 1.4 N.A. oil-immersion objective (UPLSAPO 100XO, Olympus). To obtain a dark-field illumination, the central part of the light beam is blocked. The scattered light is collected with a 0.3 N.A. objective (UPLFLN 10X2, Olympus), and subsequently focused onto a $50 \mu \mathrm{m}$ diameter pinhole before the spectrometer (AvaSpec-3648-USB2, Avantes). The effective area on the sample plane from which light was collected was estimated to be a $5 \mu \mathrm{m}$ diameter circle, hence completely encompassing the full nanostructure. Spectra were typically acquired in 5 seconds to allow the detector to accumulate enough signal. The scattering spectra are normalized by dividing the scattered light spectrum to the system response retrieved by removing the beam block (bright field illumination) to get rid of the inherent wavelength dependence of the white light source. 
Fig. 3.10 shows an example of dark-field scattering spectrum of a gold nanoparticle with $100 \mathrm{~nm}$ diameter measured by the white light dark-field microscope shown in Fig. 3.9. The measured resonance frequency $(570 \mathrm{~nm})$ has a red shift compared with simulated one $(530 \mathrm{~nm})$ due to the ignorance of the effect of glass substrate in the finite-difference time-domain (CST MWS 2013) simulation.

\subsubsection{Two-Photon Photoluminescence (TPPL) Microscopy}

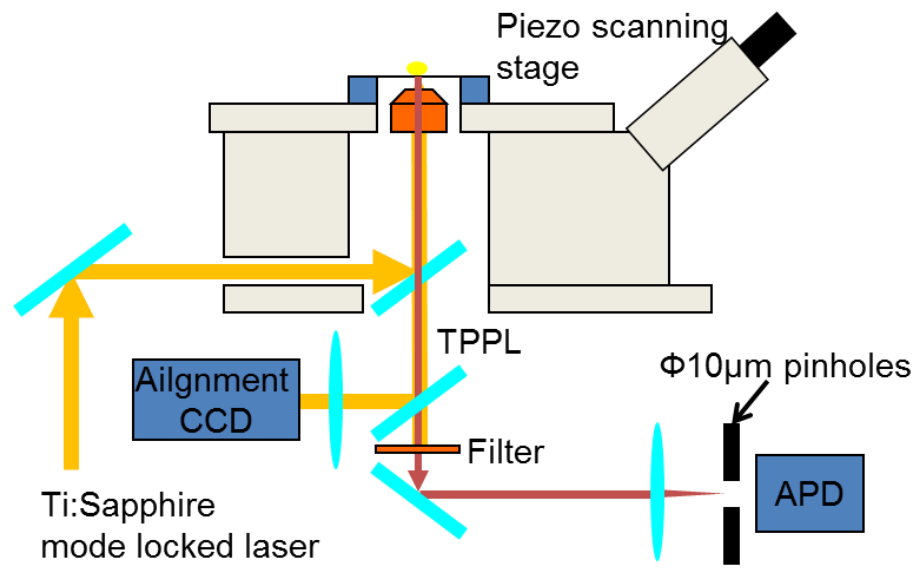

Fig. 3.11: The schematic of two photon photoluminescence (TPPL) microscopy.

In the plasmonics research field, two-photon photoluminescence (TPPL) has been widely used for mapping hot spots of gold nanostructures spatially and spectrally [55], as well as the measurement of intensity enhancement of gold bowtie optical antennas [56]. Recently TPPL was used to reveal the surface plasmon local density of states in thin single-crystalline triangular gold nanoprisms [57]. When focusing a near-infrared intense pulsed laser beam on gold, electrons in the valence $\mathrm{d}$ band absorb two photons for the transition to the conduction sp band. In this process, which is still actively studied, intraband transitions in the sp band can play a role [58]. The sensitivity of TPPL to the high electric field enhancements that occur locally in gold 


\section{Chapter 3}

nanostructures is generally attributed to its absorption of multiple photons which will be nonlinearly dependent on pump power. We use TPPL microscopy to estimate the near-field intensity enhancements of our gold nanostructures. The schematic of our TPPL microscopy is shown in Fig. 3.11.

(a)

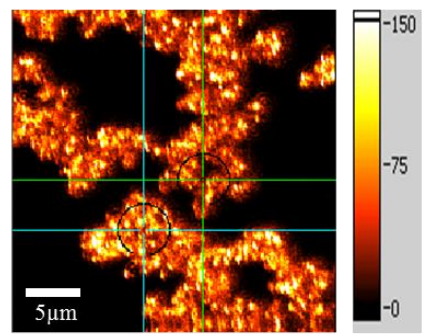

(b)

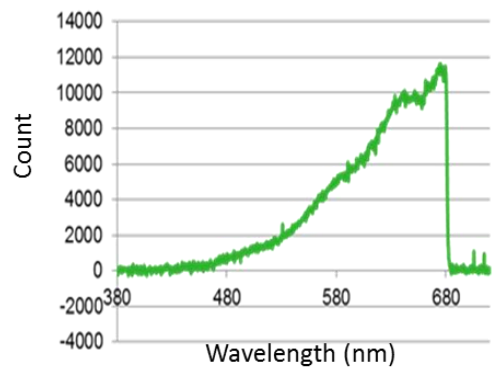

Fig. 3.12: (a) TPPL image of gold nanoparticle clusters. (b) the TPPL spectrum. The sharp cutoff around $680 \mathrm{~nm}$ is caused by the short-pass filter.

To perform TPPL confocal microscopy, we used a mode-locked laser (Micra, Coherent Inc.) with a tunable wavelength from 750 to $830 \mathrm{~nm}$. A pulse duration is around several hundred femto-seconds. The beam is sent into an inverted microscope (IX71, Olympus), and directed by a dichroic mirror (700nm dichroic short-pass filter, Edmund Optics) into the objective (UPLSAPO 100XO, Olympus). Samples are placed on a piezo scanning stage and moved relative to the focused excitation beam spot. The generated TPPL is collected by the same objective used for focusing the excitation beam, and $2^{\text {nd }}$ short pass filtered (FF01-694/SP, SEMROCK) is used to reject the excitation light. We thus only collect the visible wavelength contribution of the TPPL. The luminescent signal is focused onto a pinhole before a single-photon counting avalanche photodiode (Perkin-Elmer).

Fig. 3.12(a) shows the TPPL image of gold particles clusters with diameters around $100 \mathrm{~nm}$. The brighter spots show the higher near field intensity. Panel (b) shows the spectrum of TPPL measured by a spectrometer (AvaSpec-3648-USB2, Avantes). The broadband TPPL with a visible wavelength range of $450 \sim 680 \mathrm{~nm}$ is the result of the absorption of two near- 
(a)

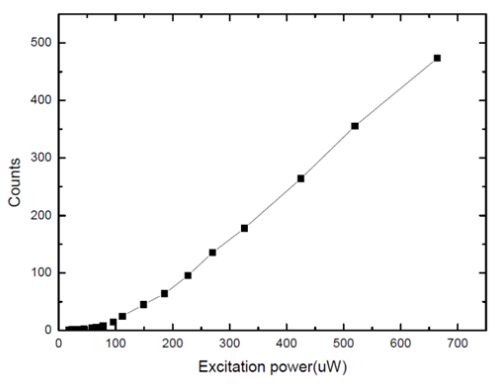

(b)

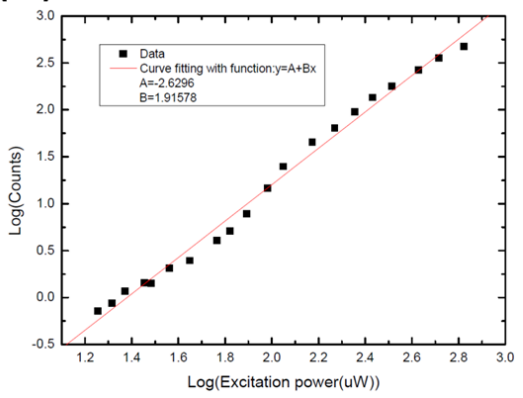

Fig. 3.13: (a) the TPPL counts v.s. excitation laser power. (b) the logarithmic plot of TPPL counts v.s. excitation laser power.

infrared photons by an electron in the valence $d$ band of gold, then the electron is excited to the conduction band, which creates a hole in the $\mathrm{d}$ band. The radiative recom-bination of the hole in the $\mathrm{d}$ band and electrons in the conduction band renders the TPPL [58].

To confirm that the luminescence signals we retrieved in Fig. 3.12(b) are indeed the result of two-photon absorption of the metal nanoparticle cluster (the nonlinear property of TPPL), we measure the relation of luminescence counts of the gold nanoparticle cluster with respect to the excitation laser power as shown in Fig. 3.13(a). The logarithmic plot of this relation is shown in Fig. 3.13(b); the red line is the linear fit of the logarithmic plot, showing the slope 1.9 , which is close to the slope 2 given by the quadratic relation: TPPL $\sim I^{2}$ or $\log (T P P L) \sim 2 \log (I)$, and $I$ is the laser intensity on the gold nanoparticle cluster. Therefore, the signals retrieved in our microscopy can be confirmed to be TPPL.

\subsubsection{Back focal plane (leakage radiation) microscopy}

An important characteristic of SPP modes is that their spatial extent is determined by the shape of metal nanostructures rather than by the optical wavelength. The instruments for the observation of SPP mode consequently 
adapted essentially to the subwavelength regime and being capable of imaging the propagation of SPPs. Usually the analysis of the subwavelength regime requires near-field scanning fiber tip [59] to frustrate and collect the evanescent components of the electromagnetic fields associated with SPPs. However, when the metal film on which nanostructures are built is thin enough (80-100 $\mathrm{nm}$ ) and when the refractive index of substratum medium (usually glass) is higher than the one of the superstratum medium (air), another possibility for analyzing SPP propagation occurs. This possibility is based on the detection of coherent leaking of SPPs through the substratum. Such a far-field optical method is called back focal plane or leakage radiation microscopy [24] [25] [26] and allows a direct and quantitative imaging of SPP propagation on thin metal films. However, in our works, we use the back focal plane microscopy on the sample formed by gold nanoparticles rather than gold films.

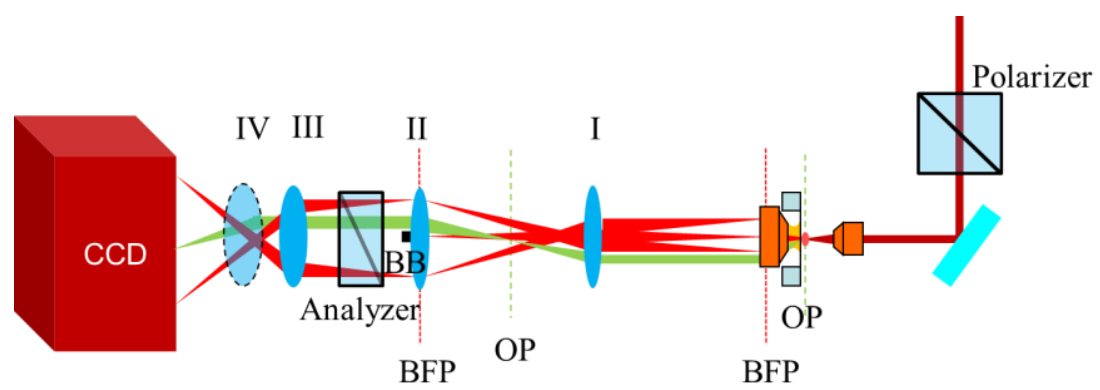

Fig. 3.14: The schematic of the back focal plane microscopy. BFP: back focal plane; BB: beam blocker; OP: object plane. The red beams represent the diffraction light from the BFP, the green beam represents the light from the OP. The zero order diffracted light and incident light are blocked by the BB.

Fig. 3.14 shows the schematic of our home-built back focal plane microscope. The photon energy of the excitation light source can be tuned from 1.3 to $2.25 \mathrm{eV}$ (550 to $950 \mathrm{~nm}$ ) with a spectral bandwidth around $2 \mathrm{~nm}$ by feeding the broadband white light source (Fianium SC400-4) into the mono-chromator (Acton SP2100-i). The excitation light is focused by a 0.3 N.A. objective (UPLFLN 10X2, Olympus) and the beam spot size is adjusted 
(a)

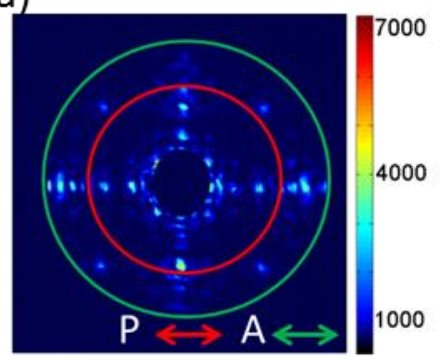

(b)

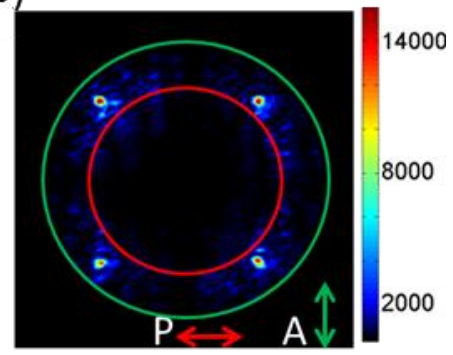

Fig. 3.15: (a), (b) diffraction pattern of a Sierpinski carpet optical antenna with an incident plane wave at $\mathrm{x}$-direction. The red double arrow is the direction of polarizer, and green double arrow is the direction of the analyzer. The red and green circles correspond to the wave vector of the light line in air and in the glass substrate, respectively.

to cover the whole sample. The diffracted light is collected by a 1.4 N.A. oilimmersion objective (UPLSAPO 100XO, Olympus). The image at the back focal plane (BFP) is relayed by lenses I, II and III in Fig. 3.14 onto the CCD (red rays). A beam block (BB) is used for blocking the incident light. With lenses I, II and IV, we can alternatively relay the image at the object plane (OP) onto the CCD (light green ray). With the polarizer and analyzer (GT10, Thorlabs) shown in Fig. 3.14, we can choose the BFP image with $\mathrm{x}$ and $\mathrm{y}$ polarization.

Fig. 3.15 shows an example of the diffraction pattern of our sample - a Sierpinski carpet optical antenna - on the back focal plane of our back focal plane microscope. The red and green circles correspond to the wave vectors of the light line in air and the glass substrate, respectively. Spots appearing between the red and green circles correspond to the evanescent waves in air but propagative waves in the glass substrate. This is a very important property of observing the near-field plasmonic mode by BFP microscopy, which will be further explained in Chapter 6 . 


\section{Chapter 4}

\section{Analysis of eigenmodes by eigen-decomposition method}

In this chapter we analyze the dipole moment structure of out-of-plane and in-plane eigenmodes of Sierpinski carpet optical antennas, and calculate Fourier spectra and far field radiation patterns of eigenmodes to identify their super- or sub-radiance properties. These analyses help us to understand the features of eigenmodes from using the self-similarity principle to construct optical antennas. Furthermore, the eigen-decomposition method introduced here will also be used in Chapter 6 to explain the specific subradiant resonance observed by back focal plane microscopy. 


\subsection{The eigen-decomposition theory of plasmonic resonances}

The analysis of the electromagnetic resonance of metallic nanostructures can be quite complicated because of the geometric complexity. For fractal optical antennas, this complexity comes from the self-similarity in the arrangement of metal nanoparticles. To understand the influence of self-similarity on the electromagnetic resonance of fractal antennas, we calculate the plasmonic eigenmodes of $0^{\text {th }}-3^{\text {rd }}$ order Sierpinski carpet optical antennas according to the eigen-decomposition method [60] [61]. The eigen-decomposition method is based on the dipolar coupling between metal nano-particles, and was first used by Markel et al [60].

\subsubsection{The coupled dipole equation}

The eigenmodes are the key to understand the collective electromagnetic resonances of metal nanoparticles. Under the coupled dipole approximation, each metal nanoparticle is assigned an electric dipole moment. With the existence of an external driving electric field $E_{\text {ext }, m}=E_{m} e^{-i \omega t}$ acting on the $m^{\text {th }}$ particle in a metal nanoparticle (MNP) cluster, the coupled dipole equation is [62] [63]:

$$
P_{m}=\alpha\left[E_{\text {ext }, m}+\sum_{n \neq m} A_{m, n} P_{n}\right],
$$

where $P_{m}$ is the dipole moment of $m^{\text {th }} \mathrm{MNP}, A_{m, n}$ is the tensor that represents the interaction matrix between a receiving dipole at $\vec{r}_{m}$ and a radiating dipole at $\vec{r}_{n} . A_{m, n}$ is defined as:

$$
\begin{gathered}
A_{m, n}\left(\vec{r} \equiv\left(\vec{r}_{m}-\vec{r}_{n}\right)\right)=k_{0}^{3}\left[B\left(k_{0}|\vec{r}|\right) \delta_{i j}+C\left(k_{0}|\vec{r}|\right) \frac{r_{i} r_{j}}{|\vec{r}|^{2}}\right] \\
A_{m, n}\left(\vec{r} \equiv\left(\vec{r}_{m}-\vec{r}_{n}\right)\right)=k_{0}^{3}\left[B\left(k_{0}|\vec{r}|\right) \delta_{i j}+C\left(k_{0}|\vec{r}|\right) \frac{r_{i} r_{j}}{|\vec{r}|^{2}}\right] \\
C(x)=\left(-x^{-1}-3 i x^{-2}+3 x^{-3}\right) e^{i x},
\end{gathered}
$$

where $k_{0}=\omega / c_{h}, c_{h}$ is the speed of light in the background medium and here we set the medium as air, and $i, j=1,2,3$ are component indices of $\overrightarrow{\mathrm{r}}$ in 
Cartesian coordinates. The term $e^{-i \omega t}$ is omitted in Eq. (4.1). If we define $A_{m, m}=1 / \alpha$, Eq. (4.1) can be written as:

$$
E_{\text {ext }, m}=A_{m, m} P_{m}+\sum_{n \neq m} A_{m, n} P_{n}
$$

This equation can be further combined to be:

$$
\sum_{n=1}^{N} A_{m, n} P_{m}=E_{\text {ext }, m},
$$

where $A_{m, n}$ is the 3 by 3 matrix defined by Eqs. (4.2), (4.3) and (4.4) and $N$ is the number of MNPs in a cluster. The polarizability $\alpha$ here is the dynamic dipole polarizability :

$$
\alpha(\omega)=i \frac{3 c_{h}^{3}}{2 \omega^{3}} a_{1}(\omega)
$$

where

$$
a_{1}(\omega)=\left.\frac{\varepsilon(\omega) J_{1}\left(k_{s} r\right) \partial_{r}\left[r J_{1}\left(k_{0} r\right)\right]-\varepsilon_{h} J\left(k_{0} r\right) \partial_{r}\left[r J_{1}\left(k_{S} r\right)\right]}{\varepsilon(\omega) J_{1}\left(k_{s} r\right) \partial_{r}\left[r H_{1}^{(1)}\left(k_{0} r\right)\right]-\varepsilon_{h} H_{1}^{(1)}\left(k_{0} r\right) \partial_{r}\left[r J_{1}\left(k_{s} r\right)\right]}\right|_{r=r_{0}}
$$

$J_{l}(x)$ is the spherical Bessel function of the first kind, $H_{l}^{(1)}(x)$ is the spherical Bessel function of the third kind (or spherical Hankel function of the first kind), and $k_{s}=\omega \sqrt{ } \varepsilon(\omega) / c_{h}$. Different from Eq. (2.15), the dynamic dipole polarizability (Eq. (4.7) and (4.8)) proposed by Doyle [64] includes the effect of radiation loss and absorption loss. Therefore it provides more accurate results for metal nanoparticles.

\subsubsection{The concept of eigenpolarizability}

We can express Eq. (4.6) as

$$
A \vec{P}=E_{\text {ext }} .
$$

To analyze the resonances of a cluster MNP, we consider the following eigenvalue problem [60]:

$$
A \vec{P}=\lambda \vec{P},
$$


where $\lambda$ and $\vec{P}=\left(\ldots, p_{m-1}, p_{m}, p_{m+1}, \ldots\right)^{T}$ are the complex eigenvalues and eigenvectors of the interaction matrix $A$. The eigen-polarizability is defined as [61]:

$$
\alpha_{e i g} \equiv \frac{1}{\lambda}
$$

which can be interpreted as the collective response function of the MNP cluster under the external electric field pattern proportional to the corresponding eigenvector $\vec{P}$. There are $3 N$ eigenmodes for a MNP cluster. For a $2 D$ MNP cluster in a plane, we can further decompose the $3 N \times 3 N$ interaction matrix A into an $N \times N$ out-of-plane $\left(p_{x}, p_{y}=0, p_{z} \neq 0\right)$ and a $2 N$ $\times 2 N$ in-plane $\left(p_{x}, p_{y} \neq 0, p_{z}=0\right)$ sub-matrix. The eigenvector $\overrightarrow{\mathrm{P}}$ gives the spatial distribution of the plasmonic eigenmode in a MNP cluster, while $\alpha_{\text {eig }}$ gives the response of this plasmonic eigenmode to the excitation wavelength.

In this work we use silver nanoparticles as monomers in the Sierpinski carpet optical antenna of all orders. The dielectric function of silver is adopted from the experimental data [22]. The interaction matrix A is a symmetric complex matrix, therefore, its eigenvectors form a complete set but are not orthogonal to each other set [60]. We can decompose a plamonic mode that is excited by any type of excitation beam, such as a plane wave, in a MNP cluster as a combination of eigenmodes calculated by the eigendecomposition theory.

\subsubsection{Localization of plasmonic eigenmode}

The eigenvector $\vec{P}$ retrieved from solving the eigenvalues of interaction matrix $A$ in Eq. (4.6) gives the spatial distribution of plasmonic eigenmode. The participation ratio (PR) of the $n^{\text {th }}$ eigenmode is defined as [65] [66]:

$$
P R(n)=\frac{1}{N} \frac{\left(\sum_{m=1}^{N}\left|p_{m}^{(n)}\right|^{2}\right)^{2}}{\left(\sum_{m=1}^{N}\left|p_{m}^{(n)}\right|^{4}\right)},
$$

which can be used as an indicator of the spatial localization properties of the $n^{\text {th }}$ eigenmode. In the case of $P R=1$, the spatial extension of this eigenmode is equal in all MNPs in the system. If $P R=1 / N$, this eigenmode is completely localized to single MNP. In general, an eigenmode can be regarded as 
localized as its $P R \ll 1$. Note that $N$ in Eq. (4.12) is equal to $1,8,64$, and 512 for the $0^{\text {th }}, 1^{\text {st }}, 2^{\text {nd }}$, and $3^{\text {rd }}$ order Sierpinski carpet optical antennas, respectively.

\subsection{Out-of-plane eigenmodes}

In this section we analyze the out-of-plane eigenmodes $(p x, p y=0, p z \neq 0$ ) of $0^{\text {th }}-3^{\text {rd }}$ order Sierpinski carpet optical antennas by the eigendecomposition method.

\subsubsection{The eigenpolarizability spectra of out-of- plane eigenmodes}

For periodic MNP arrays, the super- or sub-radiance property of plasmonic eigenmodes can be recognized by the dispersion relation. If the Bloch wave vector $k$ of an eigenmode of periodic arrays is smaller than that of the incident light, this eigenmode can couple easily with the incident light, and consequently has a higher radiation loss, therefore, this eigenmode is recognized as 'super-radiant' or 'bright'. If the Bloch wave vector $k$ of an eigenmode larger than that of the incident light, this eigenmode can hardly couple with the incident light, and consequently has lower radiation loss. This eigenmode is recognized as 'sub-radiant' or 'dark'. The sub- and superradiance property have been discussed in Chapter 2. However, for aperiodic arrays such as the Sierpinski carpet optical antenna, due to the absence of translational invariance, there does not exist a Bloch wave vector as for the periodic arrays. The super- or sub-radiance of an eigenmode is recognized by its Fourier amplitude spectrum. If the magnitude of most $k$ vectors of an eigenmode is smaller than the magnitude of the incident light, we can recognize this eigenmode is superradiant for it can be coupled with the incident light. On the other hand, if the magnitude of most $k$ vectors of an eigenmode is larger than the magnitude of the incident light, it is subradiant.

Fig. 4.1 shows the calculated imaginary part of $\alpha_{e i g} / r_{0}{ }^{3}$ of the Sierpinski carpet optical antenna up to the $3^{\text {rd }}$ order. The energy extinction of a MNP cluster by the external field is given by [61]

$$
P_{\text {loss }}=\omega \operatorname{Im}\left(\alpha_{\text {eig }}\right) \sum_{m} E_{\text {ext }, m} .
$$


Since the power loss of the system is proportion to $\operatorname{Im}\left(\alpha_{\text {eig }}\right)$ in Eq. (4.13), the spectral position of the peak of $\operatorname{Im}\left(\alpha_{e i g} / r_{0}{ }^{3}\right)$, similar to the polarizability $\alpha$ of a single MNP, indicates the resonant frequency of that eigenmode of the MNP system.
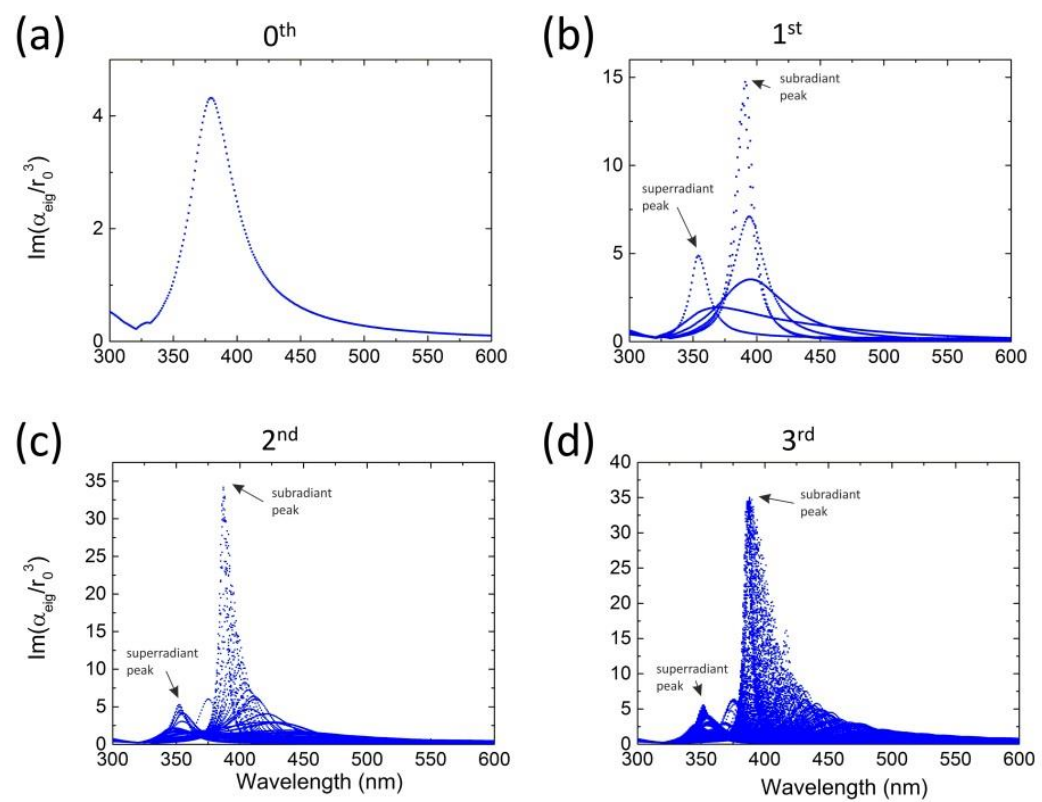

Fig. 4.1: (a) - (d) are the eigen-polarizability spectra of the out-of-plane eigenmode of $0^{\text {th }}-3^{\text {rd }}$ order Sierpinski carpet optical antenna, respectively.

Fig. 4.1(a) shows the $\operatorname{Im}\left(\alpha_{e i g} / r_{0}^{3}\right)$ of the monomer of Sierpinski carpet optical antenna, which indicates the resonance of a single silver nanoparticle at wavelength $380 \mathrm{~nm}$. We can easily connect all data points in Fig. 4.1(a) to have an eigen-polarizability spectrum curve of a monomer. When the monomer number increases in $1^{\text {st }}-3^{\text {rd }}$ order Sierpinski carpet optical antenna, the connection of data points in Fig. 4.1(b) - (d) becomes impossible due to the eigenvalue retrieving process in MATLAB (MathWorks). Fig. 4.2 shows the normalized far field radiation pattern $\left|E_{f f}\right|^{2}$ of the eigenmode in Fig. 4.1(a) 
on a unit sphere representing all the scattering directions. $E_{f f}$ is calculated by [60]

$$
E_{f f}=k^{2} \frac{e^{i k R}}{R} \sum_{j=1}^{N}\left[P_{j}-\left(s \cdot P_{j}\right) s\right] e^{-i k s \cdot r_{j}},
$$

where $r_{j}$ is the position of a MNP, $\mathrm{s}$ is the unit vector in the scattering direction and $R$ is the distance from the center of mass of a MNP to the detector with $R \gg\left|r_{i}-r_{j}\right|$ and $k R \gg 1$. Fig. 4.2 shows actually the far field radiation pattern of a single dipole located in the origin of coordinate and pointing at the $\mathrm{z}$ direction.

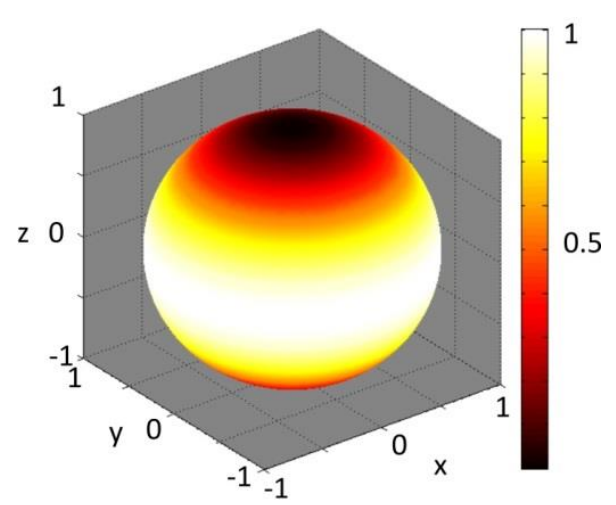

Fig. 4.2: Far field radiation pattern of the eigenmode shown in Fig. 4.1(a) at peak wavelength $380 \mathrm{~nm}$.

Fig. 4.1(b) shows the $\operatorname{Im}\left(\alpha_{\text {eig }} / r_{0}{ }^{3}\right)$ of $1^{\text {st }}$ order of Sierpinski carpet optical antenna. For the eight MNPs in this structure there should be 24 curves representing 24 plasmonic modes : 8 out-of plane and 16 in-plane. In Fig. 4.1(b) we only find 6 curves, which indicates there are two degenerate modes. 


\subsubsection{The eigenmodes of superradiant peak}

(a)

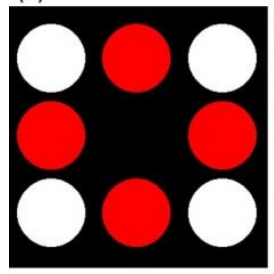

(b)

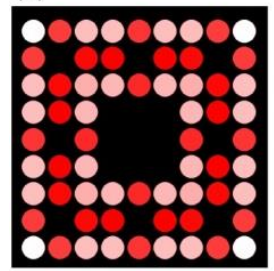

(c)

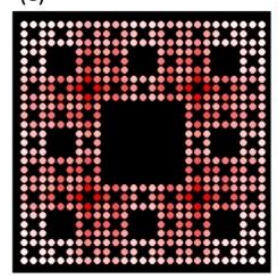

(d)

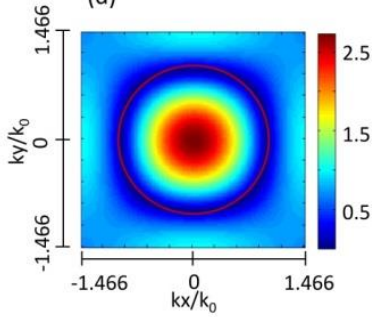

(e)
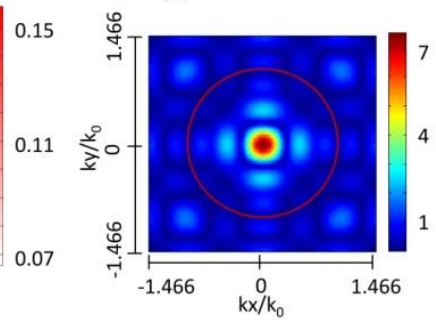

(f)

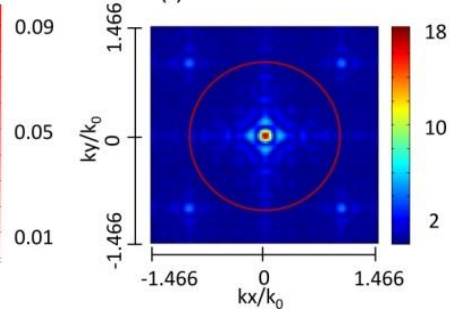

(g)

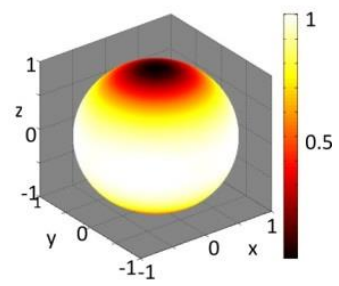

(h)

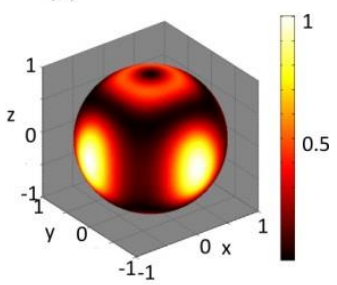

(i)

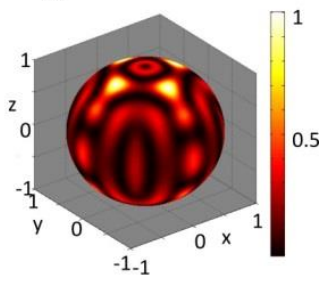

Fig. 4.3: (a) - (c) are the real part of dipole moment of eigenmodes at peaks of $355 \mathrm{~nm}$ in Fig. 4.1(b) - (d), respectively. The red color represents the $\mathrm{z}$ direction. (d) - (f) are Fourier amplitude spectra and (g) - (i) are far field radiation patterns of eigenmodes shown in (a) - (c), respectively. Note that the far field radiation patterns are normalized. The red circle in (d) - (f) represents the magnitude of the wave vector of incident light.

We notice that there are two major peaks in Fig. 4.1(b) - (d) with positions at 355 and $390 \mathrm{~nm}$, respectively. Fig. 4.3(a) - (c) show the real parts of the dipole moment of eigenmodes with maximum value of $\operatorname{Im}\left(\alpha_{\text {eig }} / r_{0}{ }^{3}\right)$ (at around 
$355 \mathrm{~nm}$ ) in Fig. 4.1(b) - (d), respectively. We find that all dipole moments of monomers are pointing in the same direction, and we can easily identify that these three eigenmodes are superradiant. To get more understanding on the superradiance property of these eigenmodes, we calculate their Fourier amplitude spectra of dipole moment by the formula

$$
\left|\widetilde{P_{z}}\left(k_{x}, k_{y}\right)\right|=\left|\int P_{z}(x, y) e^{-i\left(k_{x} x+k_{y} y\right)} d x d y\right| .
$$

Fig. 4.3(d) - (f) show the Fourier amplitude of the dipole moment of eigenmodes shown in Fig. 4.3(a) - (c), respectively. The superradiance of three eigenmodes can be easily identified: most of wave vectors are within the red circle, which represents the wave vector of incident light. Fig. 4.3(g) (i) are far field radiation patterns calculated by Eq. (4.14). As the order of Sierpinski carpet optical antenna increases, the Fourier spectra and far field radiation patterns of these three eigenmodes become more structured. The $P R$ values of the eigenmodes shown in Fig. 4.3(a) - (c) are 0.8, 0.83 and 0.49, respectively. As the order of Sierpinski carpet optical antenna increases, the eigenmodes in the sub-radiant peak get more localized, and the dipole moment distributions of higher order Sierpinski carpet optical antenna in Fig. 4.3(b) and (c) have similar features with the $1^{\text {st }}$ order eigenmode shown in Fig. 4.3(a): the four corners have weaker dipole moments and a point symmetry with respect to the center of the Sierpinski carpet optical antenna.

\subsubsection{The eigenmodes of subradiant peak}

Fig. 4.4(a) - (c) show the real part of the dipole moment of eigenmodes with maximum $\operatorname{Im}\left(\alpha_{e i g} / r_{0}{ }^{3}\right)$ (at $391 \mathrm{~nm}$ ) in Fig. 4.1(b) - (d), respectively, and Fig. 4.4(d) - (f) are their Fourier amplitude spectra of the dipole moment calculated by Eq. (4.15), and Fig. 4.4(g) - (i) are their far field radiation patterns calculated by Eq. (4.14). Fig. 4.4 (a) shows two degenerate eigenmodes. Different from the eigenmodes in the superradiance peak shown in Fig.4.3(a) - (c), eigenmodes in Fig. 4.4(b) and (c) have a symmetry on anti-phased dipoles (blue and red squares): the dipole moments on their diagonal lines and $\mathrm{x}, \mathrm{y}$-axis lines are zero (white squares), and anti-phase dipoles are symmetric with respect to these four lines, too. Due to the highly symmetric anti-phase dipoles in these eigenmodes, the radiation damping is reduced and consequently these eigenmodes possess a higher quality factor compared with superradiant eigenmodes shown in Fig. 4.3(a) - (c). From 
their Fourier spectra in Fig. 4.4(d) - (f), we can see that as the order of the Sierpinski carpet optical antenna increases, the spectra become more structured but still distribute in the same areas outside of the red circle. Fig. $4.4(\mathrm{~g})$ - (i) show that far field radiation patterns are also getting more structured. Contrary to the superradiant eigenmodes discussed in Fig. 4, we call this peak the 'subradiant peak'. The $P R$ value for three eigenmodes shown in Fig. 4.4(a) - (c) are 0.68, 0.58 and 0.47, respectively, which shows, similar to superradiant eigenmodes shown in Fig. 4.3(a) - (c), eigenmodes get more localized as the order increases. 
(a)
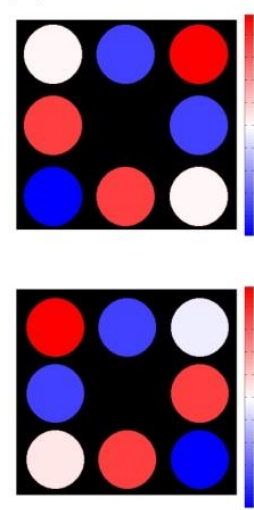

(b)

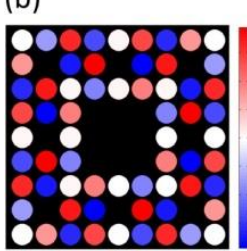

(c)

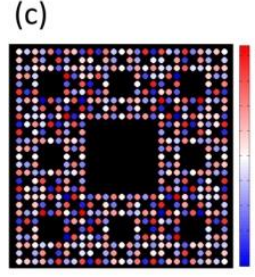

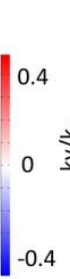

స్తి

(d)
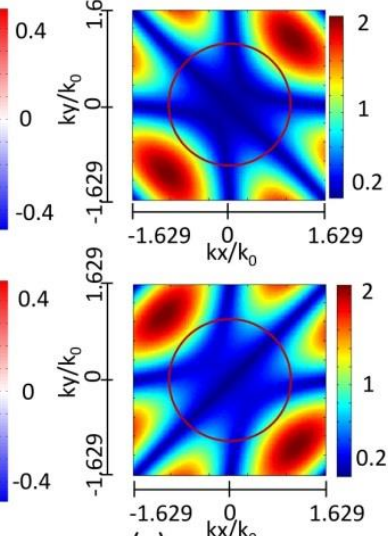

(e) $\mathrm{kx} / \mathrm{k}_{0}$
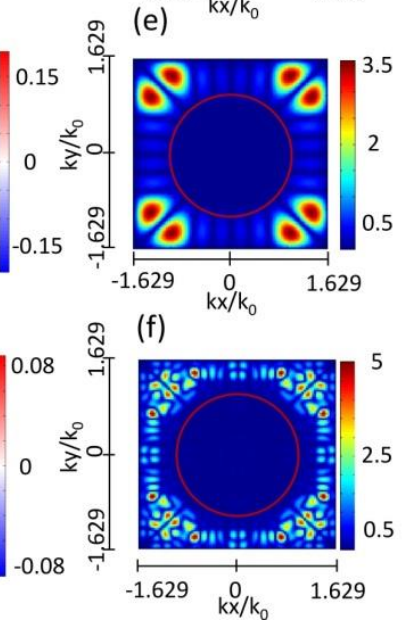

(g)
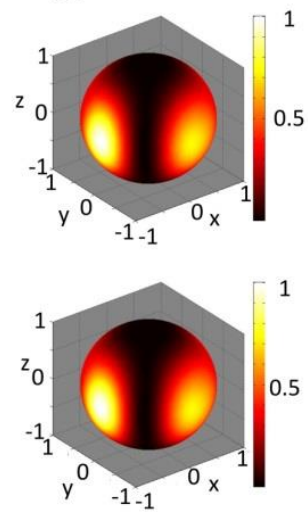

(h)

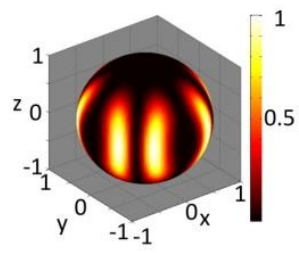

(i)

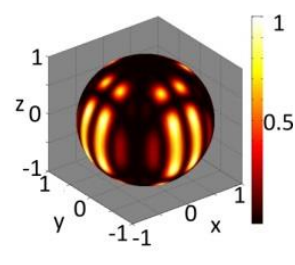

Fig. 4.4: (a) - (c) are the real part of dipole moment of eigenmodes at peaks of $391 \mathrm{~nm}$ in Fig. 4.1(b) - (d), respectively. The red and blue color represent the $\mathrm{z}$ and $-\mathrm{z}$ direction. (d) - (f) are Fourier amplitude spectra and (g) - (i) are far field radiation patterns of (a) - (c), respectively. The red circle in (d) - (f) represents the magnitude of the wave vector of incident light. 


\subsection{In-plane eigenmodes}

In this section we analyze the in-plane eigenmodes $(p x, p y \neq 0, p z=0)$ of $0^{\text {th }}-3^{\text {rd }}$ order Sierpinski carpet optical antennas by the eigen-decomposition method.

\subsubsection{The eigenpolaribility spectra of in-plane eigenmodes}

Fig. 4.5(a) - (d) show the calculated $\operatorname{Im}\left(\alpha_{e i g} / r_{0}{ }^{3}\right)$ of in-plane $\left(p_{x}\right.$ and $p_{y} \neq 0, p_{z}$ $=0$ ) eigenmodes of $0^{\text {th }}-3^{\text {rd }}$ order Sierpinski carpet optical antennas. Fig. 4.5(a) is a degenerate curve which is exactly the same as the curve shown in Fig. 4.1(a). Fig. 4.6(a) and (b) show the far field radiation patterns of these two degenerate eigenmodes in Fig. 4.5(a) and are exactly the far field radiation patterns of a single dipole pointing in $\mathrm{x}$ and $\mathrm{y}$ direction, respectively.

Due to the degeneracy in eigenmodes, Fig. 4.5(b) shows only 12 different curves representing 16 (4 degenerate eigenmodes) in-plane plasmonic eigenmodes. Contrary to the eigenpolarizability spectra shown in Fig. 4.1, we find that as the order increases, many new peaks arise, while the peaks at 364 and $385 \mathrm{~nm}$ in Fig. 4.5(b) - (d) remain at the same position. Fig. 4.7 is the vector plot of the real part of the dipole moment $\left(p_{x}, p_{y}\right)$ of the inplane plasmonic eigenmode, (a) - (c) show the eigenmodes of $1^{\text {st }}-3^{\text {rd }}$ order Sierpinski carpet optical antenna with maximum $\operatorname{Im}\left(\alpha_{e i g} / r_{0}{ }^{3}\right)$ value at $385 \mathrm{~nm}$, respectively. 


\section{Analysis of eigenmodes by eigen-decomposition method}

(a)

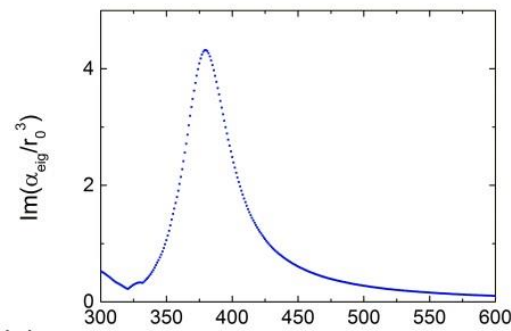

(c)

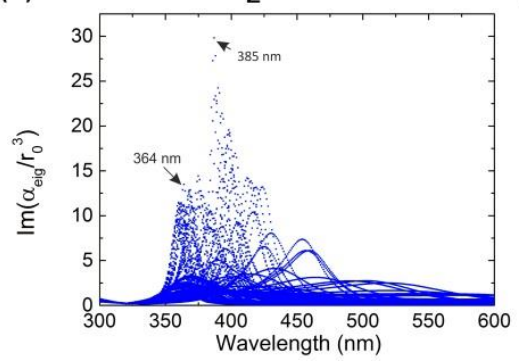

(b)

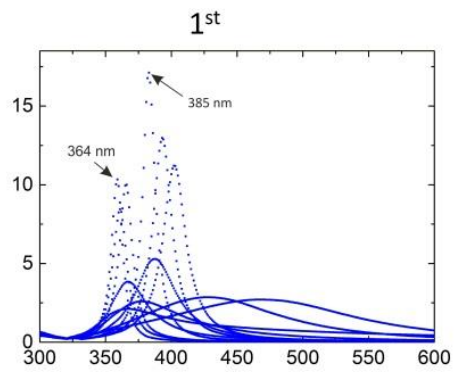

(d)

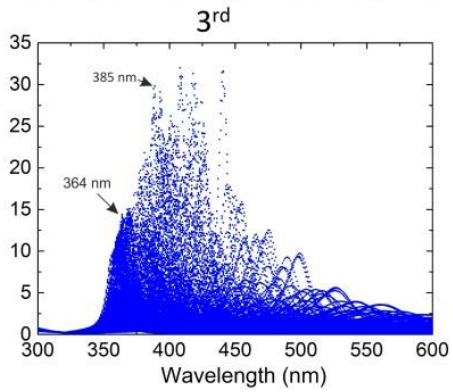

Fig. 4.5: (a) - (d) are the eigenpolarizability spectra of the in-plane eigenmode of $0^{\text {th }}-3^{\text {rd }}$ order Sierpinski carpet optical antenna, respectively.
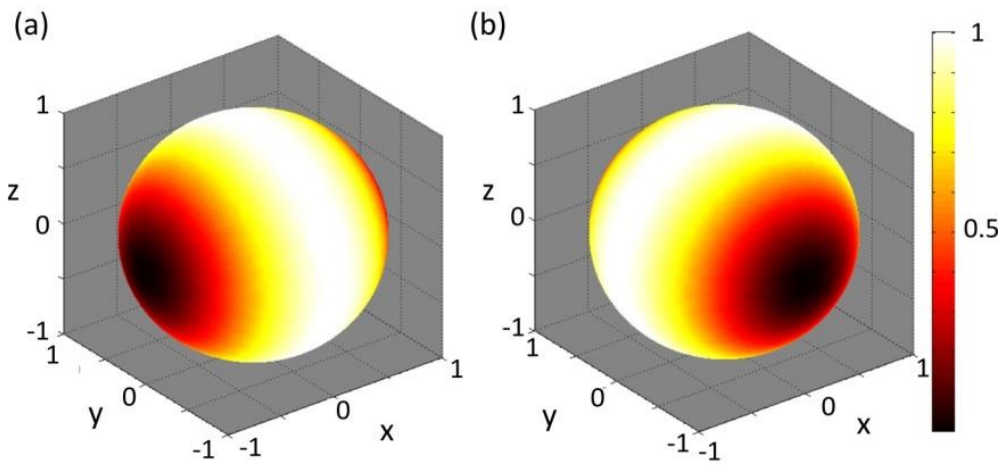

Fig. 4.6: (a), (b) are the far field radiation patterns of two degenerate modes in Fig.4.5(a). 


\subsubsection{The self-similarity in the structure of dipole moment}

In Fig. 4.7(a), we find that the anti-phase dipole moments have point symmetry with respect to the center of the $1^{\text {st }}$ order Sierpinski carpet optical antenna. Fig. 4.7(d) and (g) are Fourier spectra of the dipole moment of the eigenmode calculated by Eq. (15) with $P_{z}(x, y)$ replaced by $P_{x}(x, y)$ and $P_{y}(x, y)$, respectively. Most wave vectors of the eigenmode are outside of the circle of light line, showing its subradiance property. Fig. 4.7(j) shows the far field radiation pattern calculated by Eq. (4.14).

Fig. 4.7(b) and (c) are vector plots of the real part of the dipole moment $\left(p_{x}, p_{y}\right)$ of eigenmodes with maximum $\operatorname{Im}\left(\alpha_{e i g} / r_{0}{ }^{3}\right)$ at $385 \mathrm{~nm}$ in Fig. 4.5(c) and (d), respectively. From the vector plots we find that the dipole moment structures of these eigenmodes also possess self-similarity. In Fig. 4.7(b), the vector plot shows that $1^{\text {st }}$ order subunits have a similar dipole moment structure as the $1^{\text {st }}$ order shown in Fig. 4.7(a): the anti-phase dipole moment has a point symmetry with respect to the center of each $1^{\text {st }}$ order subunit. Fig. 4.7(c) also shows similar dipole moment structures in its $1^{\text {st }}$ order subunits. In Fig. 4.7(a) - (c), all eigenmodes have a point symmetry of the anti-phase dipole moment with respect to the center, which reduces of radiation damping as the order increases. As the order increases from $2^{\text {nd }}$ to $3^{\text {rd }}$, we find the dipole moment concentrate more on some parts of monomers, which means eigenmodes getting more localized as the order increases. The calculated PR value of these three eigenmodes are $0.97,0.62$ and 0.23 , respectively. From the Fourier spectra of $p_{x}$ and $p_{y}$ in Fig. 4.7(d) - (f) and (g) (i), we notice that wave vectors distribute in the same $\mathrm{k}$ space region and become more structured and discretized as the order increases.

Fig. 4.8(a) - (c) are the vector plots of the real part of dipole moment of the in-plane eigenmode which has maximum $\operatorname{Im}\left(\alpha_{\text {eig }} / r_{0}{ }^{3}\right)$ value at $364 \mathrm{~nm}$ in Fig. 4.5(b) - (d), respectively. In Fig. 4.8(b) and (c), we see that the dipole moment structure of the $1^{\text {st }}$ order subunits have the same symmetry as the dipole moment structure of $1^{\text {st }}$ order Sierpinski carpet optical antenna shown in Fig. 4.8(a), in which the anti-phase dipole moment is symmetric with respect to the center, which reduces the radiation loss. We can see that, in Fig. 4.8(c), the dipole moments distribute in inner and outer $1^{\text {st }}$ order subunits, which shows more localized property as the order increases. The $P R$ value of 
eigenmodes shown in Fig. 4.8(a) - (c) are 0.94, 0.8 and 0.62, respectively. From Fig. 4.8(d) - (f), as the eigenmodes shown in Fig. 4.7(a) - (c), most of their wave vectors are smaller than that of the incident light which is represented by the red circle. It shows their sub-radiance property. With the increase of order, the Fourier spectra (d) - (i) and far field radiation patterns (j) - (l) become more structured. 


\section{Chapter 4}

(a)
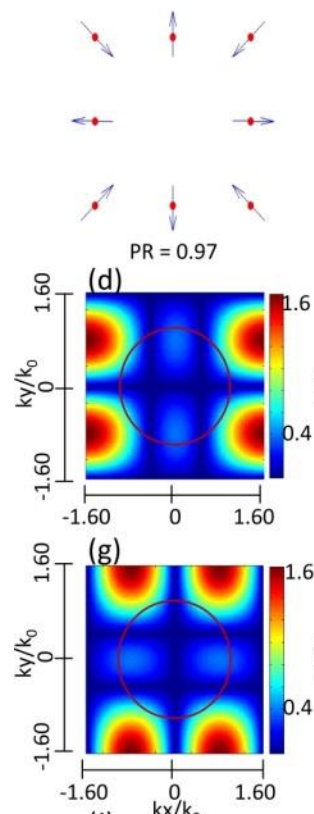

(j)

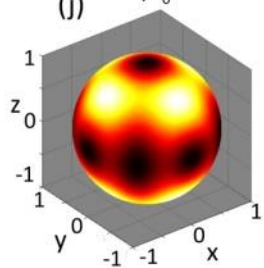

(b)

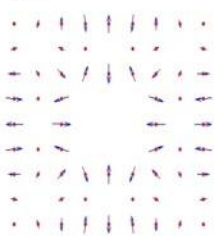

$P R=0.62$
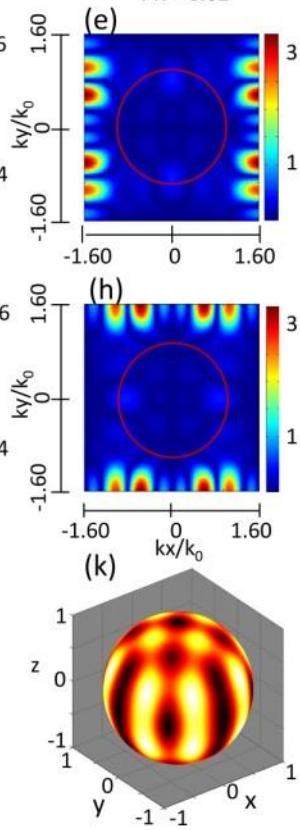

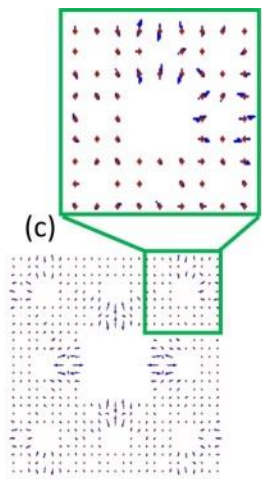

$P R=0.23$
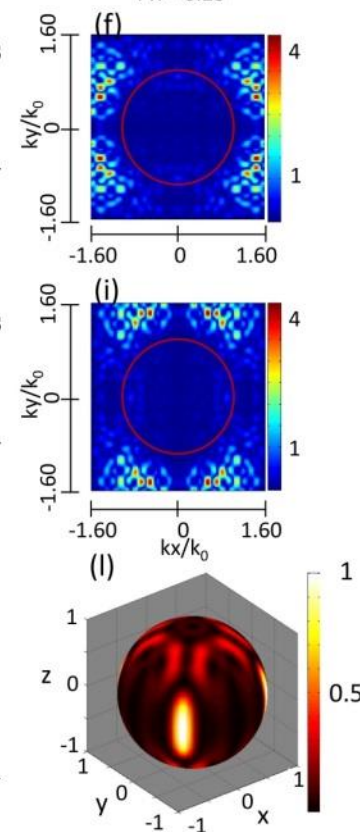

Fig. 4.7: (a) - (c) are the real parts of the dipole moment of eigenmodes at the peak $385 \mathrm{~nm}$ in Fig. 4.6(b) - (d), respectively. (d) - (f) are the Fourier spectra of dipole moment $p_{x}$ and (g) - (i) are the Fourier spectra of dipole moment $p_{y}$ of eigenmodes in (a) - (c), respectively. And (j) - (l) are the far field radiation patterns of (a) - (c), respectively. 
(a)

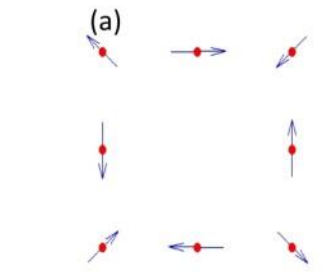

(b)

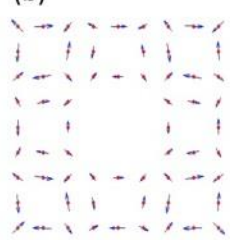

(c)

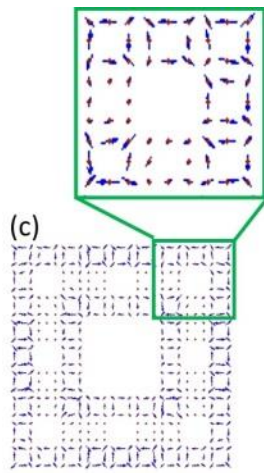

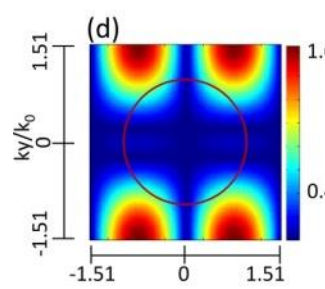
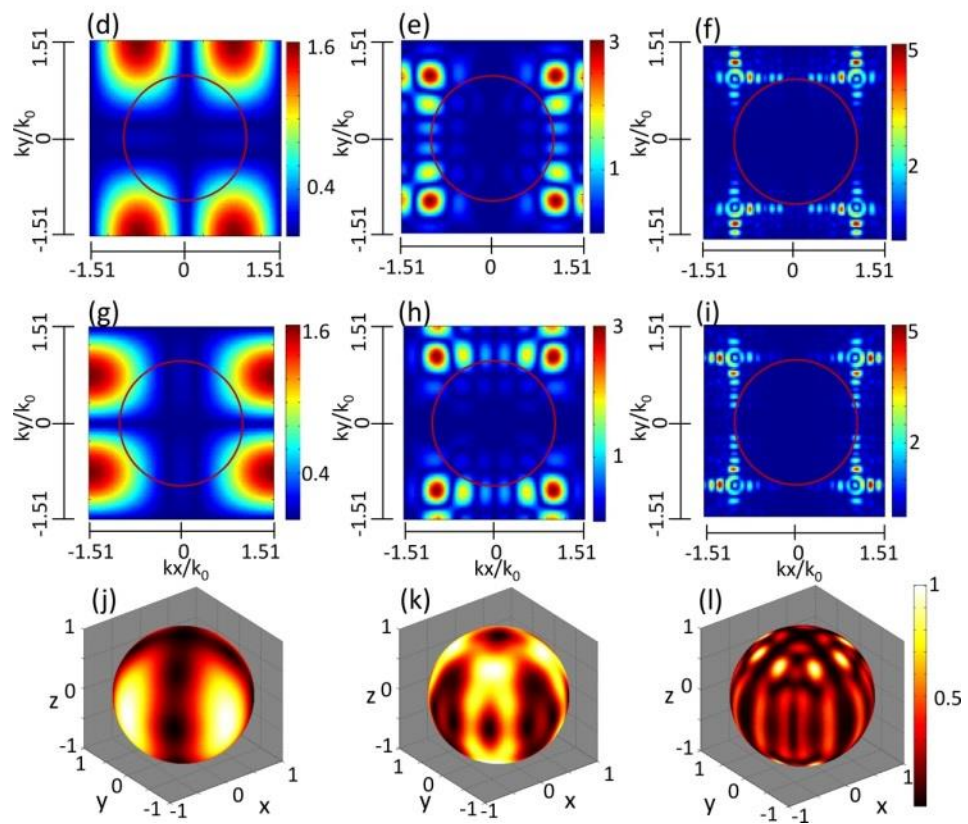

Fig. 4.8: (a) - (c) are the real part of dipole moment of eigenmode at peak of $364 \mathrm{~nm}$ in Fig. 4.6 (b) - (d), respectively. (d) - (f) are the Fourier spectra of dipole moment $p_{x}$ and (g) - (i) are the Fourier spectra of dipole moment $p_{y}$ of eigenmodes in (a) - (c), respectively. And (j) - (l) are the far field radiation patterns of eigenmodes shown in (a) - (c), respectively. 


\subsection{The trajectory plot of wavelength - far field radiation energy - PR value}

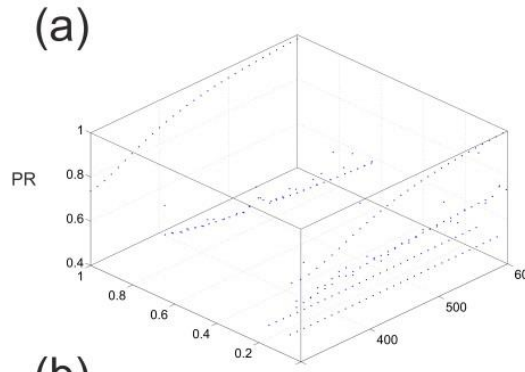

(b)
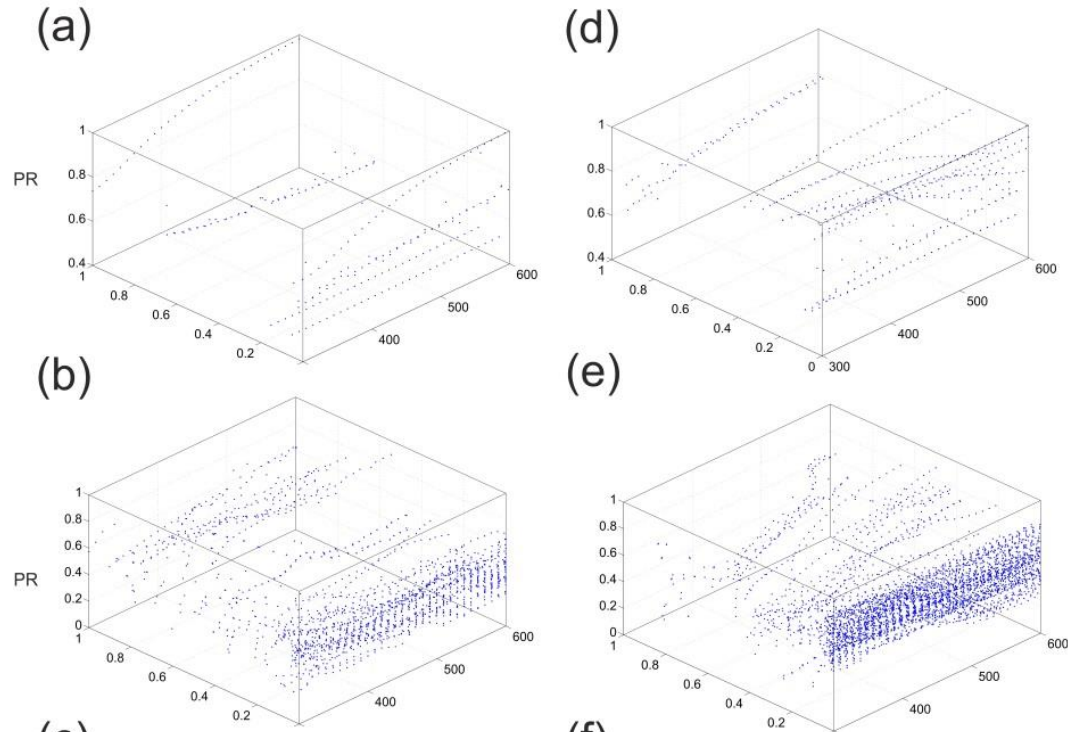

(c)
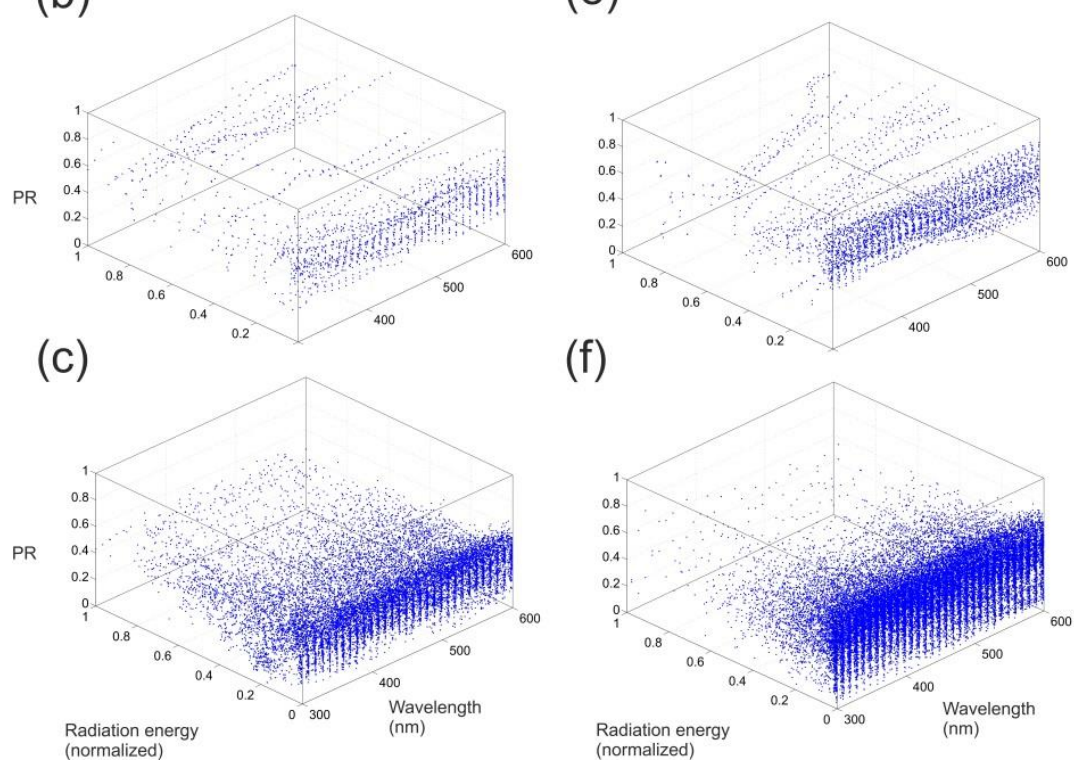

(f)

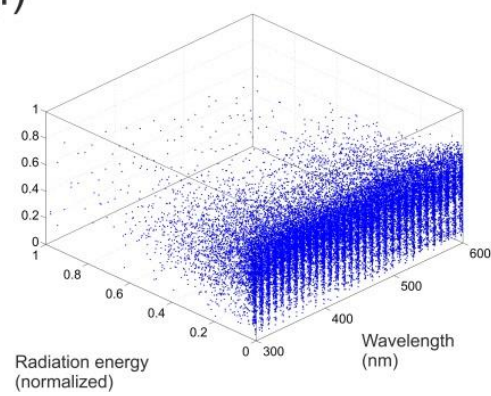

Fig. 4.9: (a) - (c) show the trajectory plot of [wavelength (every $10 \mathrm{~nm}$ ), radiation energy (normalized), $P R]$ for out-of-plane eigenmodes of $1^{\text {st }}-3^{\text {rd }}$ order Sierpisnki carpet optical antenna. (d) - (f) are the trajectory plot for the in-plane eigenmodes.

Fig. 4.9(a) - (c) show trajectory plots of wavelength, radiation energy (normalized), and $P R$ of the out-of-plane eigenmodes for the $1^{\text {st }}-3^{\text {rd }}$ order 
Sierpinski carpet optical antennas. Similarly, Fig. 4.9(d) - (f) show the trajectory plots for the in-plane eigenmodes. The normalized far field radiation energy can indicate the sub- or super-radiance of eigenmodes. When $P R \sim 1$, it is a superraidant eigenmod e; while $P R \sim 0$ indicates the eigenmode is subradiant. The trajectory map in Fig. 4.9 gives us statistical information on the sub- and super-radiance of eigenmodes with respect to the wavelength and $P R$ value. The $P R$ value indicates the level of localization of an eigenmode. In Fig. 4.9, we find that eigenmodes possess lower $P R$ value as the order increases, which means higher order Sierpinski carpet optical antennas can have stronger localized eigenmodes.

(a)

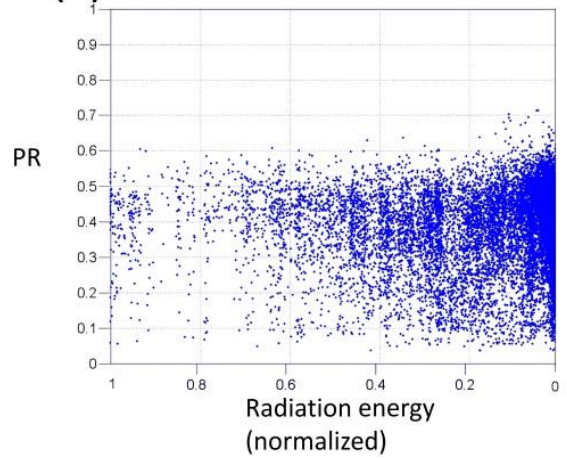

(b)

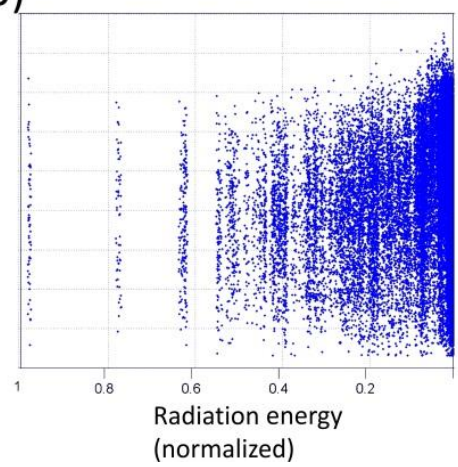

Fig. 4.10: (a) the projection of [Radiation energy, $P R$ ] of Fig. 4.9(c). (b) the projection of [Radiation energy, $P R$ ] of Fig. 4.9(f).

From Fig. 4.9(a), (b), it can be seen that the $P R$ value of some curves changes with the wavelength. This shows that the dipole moment structure may change with resonance on or off. Further, it is can be seen that most of the eigenmodes are subradiant, as most of the points in the trajectory plots have normalized radiation energy $\sim 0$. For $3^{\text {rd }}$ order structures in panels (c) and (f), this situation is even clearer. This agrees with the discovery in Ref. [67] that for most of eigenmodes of a random metal nanostructure are subradiant. Fig. 4.10(a) and (b) are the projection plane of [radiation energy, $P R]$ of the out-of-plane and in-plane eigenmodes of $3^{\text {rd }}$ order Sierpinski carpet optical antenna, respectively. From Fig. 4.10 we can see more clearly 
that most eigenmodes are subradiant and the localization property is unrelated to the sub- or super-radiance property of eigenmodes.

\subsection{Conclusion}

The eigen-decomposition theory is adopted to calculate the dipole moment structure of eigenmodes of the $0^{\text {th }}-3^{\text {rd }}$ order Sierpinski carpet optical antenna. For out-of-plane eigenmodes, there exists obviously a superradiant and a subradiant peak in the eigenpolarizability spectra. The spectral positions of the two peaks remain the same as the order increases from $1^{\text {st }}$ to $3^{\text {rd }}$. For inplane eigenmodes, it does not show clear and separate super- and sub-radiant peaks in eigenpolarizability spectra as the out-of-plane eigenmodes. The selfsimilarity of the dipole moment structure of eigenmodes are found and discussed. For out-of-plane eigenmodes, the self-similarity of eigenmodes at super- and sub-radiant peaks is discussed. As the order of Sierpinski carpet optical antenna increases, the eigenmodes in super-and sub-radiant peaks become more localized. For the in-plane eigenmodes, due to the complicated eigenpolarizability spectra, only two peaks are chosen to be analyzed. These two eigenmodes remain at the peak of 385 and $364 \mathrm{~nm}$ of eigenpolarizability spectra as the order increases. The self-similarity of dipole moment of these two eigenmodes is discussed. And they become more localized as the order increases. However, there is no evidence that other eigenmodes have this self-similarity in their dipole moment structure because the complexity of eigenpolarizability spectra renders the difficulty of analysis of other eigenmodes. These analyses helps us to understand the influence of selfsimilarity property of the fractal optical antenna on plasmonic eigenmodes, especially when the order of fractal optical antenna increases, the symmetry of anti-phased dipole moment of some eigenmodes can be predicted. 


\section{Chapter 5}

\section{Characterization at visible and near-infrared wavelengths}

In this chapter we investigate the far-field scattering spectrum and near-field intensity enhancement of the Sierpinski carpet gold nanostructure, both experimentally and numerically. In addition, we employ a pseudo-random and a periodic gold nanostructure to explore the influence of morphology on the optical properties of these large arrays of gold particles. Scattering spectra of the gold nanostructures were obtained by white-light dark field microscopy, and two-photon photoluminescence (TPPL) microscopy was used to visualize the near-field intensity enhancement of three gold nanostructures. 


\subsection{Three morphologies of broadband optical antennas}

It has been shown that metal nano-rods, dipole and bowtie optical antennas can be fabricated with dimensions down to several tens of nanometer, allowing precise tuning of optical properties such as the resonant wavelength [19] [18]. To create optical antennas with a broadband response, pseudorandom, periodic and aperiodic metal nanostructures have recently been studied [68] [69] [70] [71]. Here we investigate the optical properties of metal particle nanostructures based on Sierpinski carpet, pseudo-random and periodic geometries. We will discuss the schematic and fabrication of these three nanostructures in this section.

\subsubsection{Sample schematics}

(a)

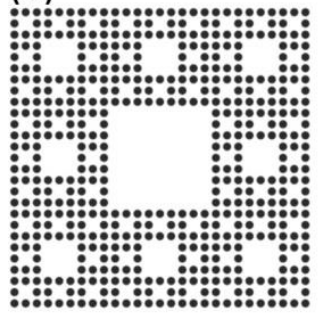

(d)

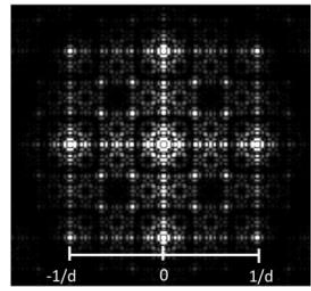

(b)

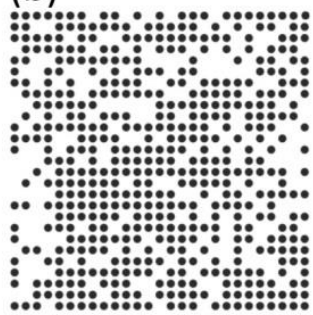

(e)

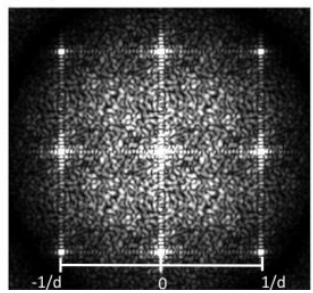

(c)

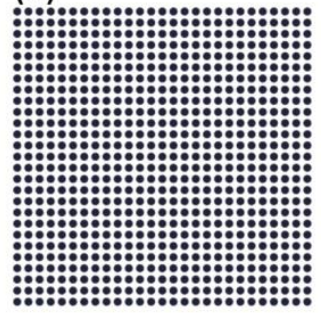

(f)

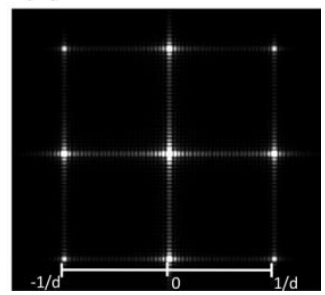

Fig. 5.1: Schematics (a) - (c) and their Fourier spectra amplitude (d) - (f) of Sierpinski carpet, pseudo-random and periodic structures. In the scale bar, $\mathrm{d}$ is the center-to-center monomer distance of the Sierpinski carpet, pseudo-random and periodic structures. The ratio of the monomer diameter to the distance $d$ is $8: 11$. 
The geometric patterns of the three samples are shown in Fig. 5.1, both in real space (a) - (c) and k-space (d) - (f). A total of 512 individual monomers are used to create the Sierpinski carpet pattern and pseudo-random distribution shown in (a) and (b), respectively, corresponding to $70 \%$ of the available sites in the $27 \times 27$ matrix. For the periodic structure (c), all 729 lattice sites are covered. Of course, the pseudo-random pattern shown in Fig. 5.1(b) is just one realization (out of $>10100$ possible configurations!) in which the 512 monomers are distributed randomly on the lattice. It is chosen arbitrarily for its lack of self-similarity relative to the Sierpinski carpet and periodic patterns, and thereby affords an opportunity for comparison. Nevertheless, the reader is cautioned against generalizing the results from what might just be lucky chance and not characteristic of all random configurations.

To better compare the three morphologies we switch to k-space by calculating their spatial Fourier transforms. The Fourier spectra amplitudes are plotted in Fig. 5.1(d) - (f), directly below their corresponding patterns (a) - (c). Primary peaks in the Fourier spectra amplitude image of the three configurations reflect the square lattice structure, as best seen in the fully periodic case (f). In the Sierpinski carpet morphology (d), besides the primary peaks, other distinct peaks are observed, corresponding to regular periodicities present in the carpet. With the increase of the iterative order of the Sierpinski carpet structure, the Fourier spectra amplitude will have more distinct points (shown in the Fig. 5.2). In contrast, the reciprocal vectors in the pseudo-random structure (e) are more evenly and continuously distributed as compared with that from the Sierpinski carpet structure. The symmetry in the Sierpinski carpet morphology results in a regular and highly symmetric distribution of its reciprocal vectors, filling in the regions between the primary peaks of the periodic morphology. The pseudo-random morphology only shows a point symmetry with respect to the origin.

\subsubsection{Sample fabrication}

Fig. 5.3 shows fabrication results of the Sierpinski carpet, pseudo-random and periodic gold nanostructures on a single crystalline gold flake. All nanostructures have the same monomer size of $80 \pm 8 \mathrm{~nm}$ diameter and $40 \pm$ $5 \mathrm{~nm}$ thickness; the gap distance between monomers is $30 \pm 11 \mathrm{~nm}$. Monomer numbers for the Sierpinski carpet and pseudo-random 


\section{Chapter 5}

(a)

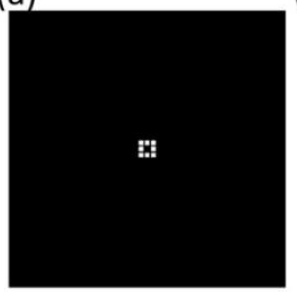

(d)

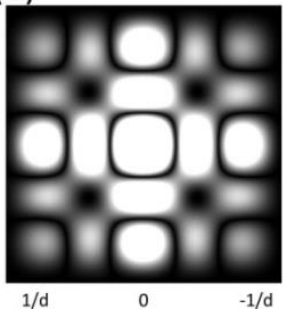

(b)

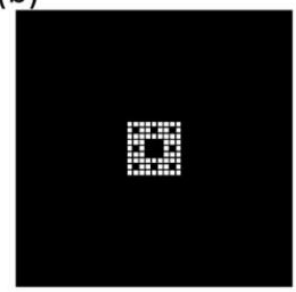

(e)

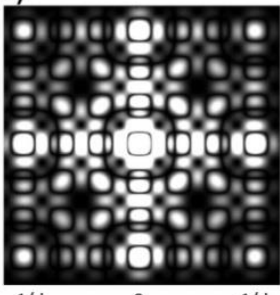

(c)

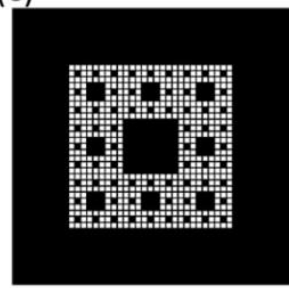

(f)

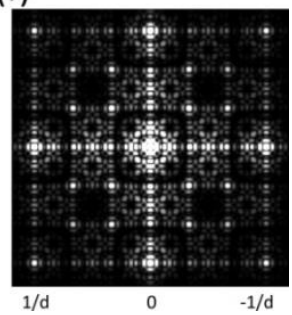

Fig. 5.2: (a) - (c) Sierpinski carpet of $1^{\text {st }}, 2^{\text {nd }}$ and $3^{\text {rd }}$ order, respectively. (d) - (e) are Fourier amplitude of (a) - (c), respectively. $d$ is the minimum distance between monomers.

(a)

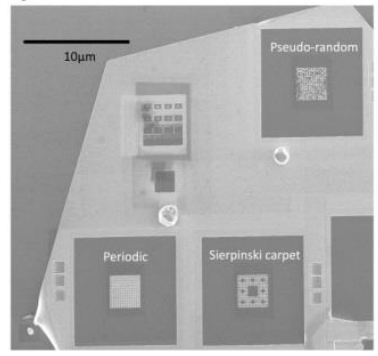

(b)

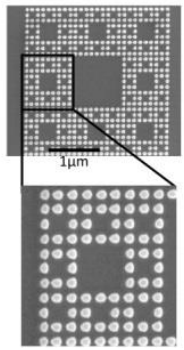

(c)

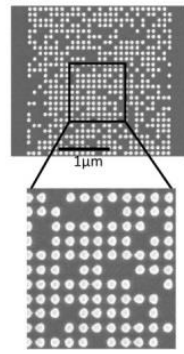

(d)

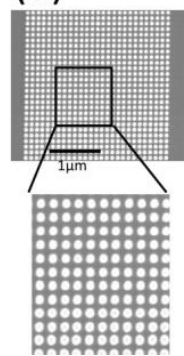

Fig. 5.3: SEM images of samples fabricated by FIB milling: (a) fabrication of Sierpinski carpet, pseudo-random and periodic nanostructures on a single crystalline gold flake, (b) the Sierpinski carpet nanostructure, (c) the pseudo-random nanostructure and (d) the periodic nanostructure. The monomer size and gap distance between monomers for all samples are $80 \mathrm{~nm}$ diameter, 40 $\mathrm{nm}$ height, and $30 \mathrm{~nm}$ gap distance. 
nanostructures are both 512, corresponding to $70 \%$ of available sites in the 27 x 27 matrix; in the periodic nanostructure all 729 sites are occupied. The monomer size was chosen such that the fabricated nanostructures exhibit optical response in the spectral detection range of our acquisition system (400-900 nm).

\subsection{Far-field scattering property}

Numerical results were obtained using finite difference time domain (FDTD, Lumerical Solutions Inc.) method. For this a total-field scattered-field (TFSF) plane wave source with normal incidence on the gold nanostructures was used as an excitation source. The mesh size in the simulation is $2 \mathrm{~nm}$ and we use "conformal variant 0 " for the mesh refinement. The structures were composed of gold cylinders, the dielectric function of gold and glass are from references [22] and [28], respectively.

To assess the broadband response of the Sierpinski carpet optical antenna, we determine its scattering spectrum in the visible and near-infrared wavelength regions. In Fig. 5.4, both measurement (a) and simulation (b) results are presented. Experimental results reveal a broad signal that peaks at $\sim 660 \mathrm{~nm}$, which is close to the result found in the numerical simulations. To determine whether this spectral response is due to the Sierpinski carpet morphology or just due to many monomers in close proximity, we also explore the pseudo-random and periodic structures. The former shows little difference, however the scattering spectrum of the periodic nanostructure is red-shifted, now peaking at $\sim 690 \mathrm{~nm}$. Although all three morphologies produce a broadband scattering response, the periodic one shows the lowest efficiency for the measured spectrum, while the simulation indicates much higher scattering efficiency than the Sierpinski carpet and pseudo-random nanostructures. This discrepancy arises from the exclusion of the 0th diffraction in the dark field configuration of experimental setup, whereas the simulation includes all scattered light. With higher monomer number ( 729 for the periodic and 512 for the Sierpinski carpet and pseudo-random nanostructures), the periodic nanostructure has higher 0th diffraction, therefore, the periodic one shows higher scattering efficiency in the simulation result. 


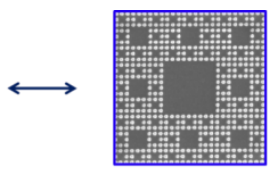

(a)

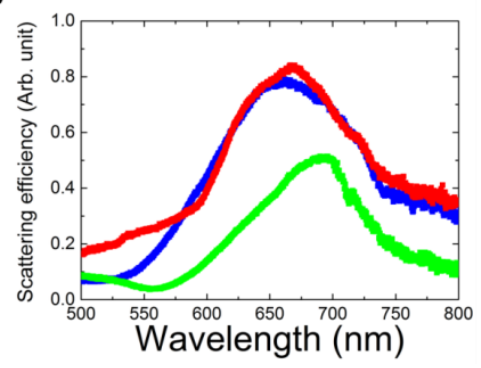

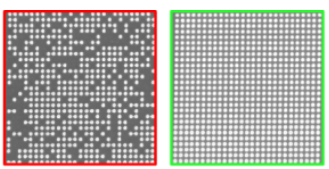

(b)

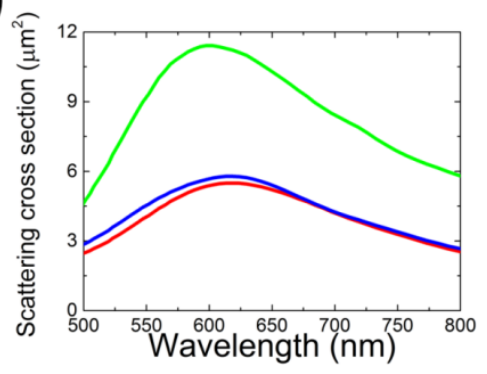

Fig. 5.4: Measurement results (a) and numerical simulations (b) of the scattering efficiency of the Sierpinski carpet (blue), pseudo-random (red) and periodic (green) gold nanostructures. The double arrow indicates the polarization direction of the incident light. After subtraction of the background scattering signal arising from the glass substrate near the nanostructures, the scattering spectra are normalized by the bright-field illumination signal in order to remove the inherent wavelength dependence of the illuminating lamp.

\subsection{Near-field intensity measurements}

In this section we will discuss the near-field intensity enhancement of the Sierpinski carpet, pseudo-random and periodic gold nanostructures. The TPPL microscopy is adopted to estimate the near-field intensity factor $\alpha$ of three nanostructures. The simulated result of $\alpha$ by the FDTD method is discussed in the end of this section. 


\subsubsection{The two photon photoluminescence images of samples}

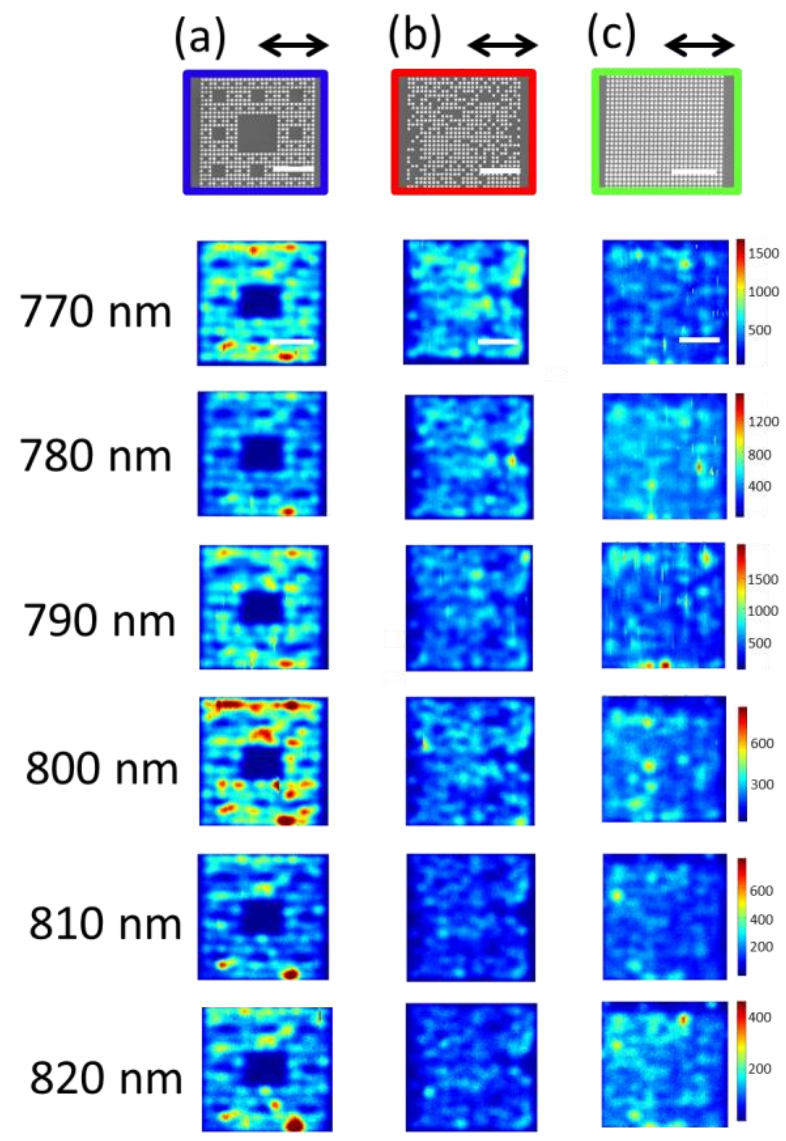

Fig. 5.5: SEM and TPPL images of (a) Sierpinski carpet, (b) pseudo-random and (c) periodic gold nanostructures at an excitation wavelength of 770, 780, 790, 800, 810 and $820 \mathrm{~nm}$. The double arrow indicates the direction of polarization of the excitation beam. The color bar indicates the number of counts collected for a scanning rate of $2.5 \mathrm{msec} / \mathrm{pixel}$. The excitation power is around $1 \mathrm{~mW}$ for all samples. All white scale bar are $1 \mu \mathrm{m}$. 
To determine the near-field intensity enhancement of the Sierpinski carpet optical antenna, we record TPPL images as a function of excitation wavelength, which are further benchmarked by comparison to signals generated by the pseudo-random and periodic morphologies. As can be seen in Fig. 5.5, the intensity distribution of the TPPL counts roughly matches the morphology of the nanostructure; lower count numbers are generally correlated with a region with a lower density of monomers. Apart from this structural signature, a few "hot spots" are visible, most notably in the TPPL images of the Sierpinski carpet and periodic nanostructures. These hot spots are not symmetrically distributed as would be expected from the symmetry of the geometric patterns. In addition, the positions of the hot spots vary for different excitation wavelengths. Hence, we attribute these hot spots as arising from small defects due to the inevitable fabrication imperfections in FIB milling.

\subsubsection{The estimation of near field intensity by TPPL images}

To characterize the overall TPPL intensity generated by the nanostructures and determine the extent to which the hotspots contribute therein, we perform a histogram analysis of each image by plotting TPPL counts per pixel. As can be seen in Fig. 5.6, the signals arising from regions containing hot spots are found in the long tail on the right side of the histogram, and do not influence the central position and width of the main curve under which most intensity is found. Fig. 5.6(b) shows the center and full width at half maximum (FWHM) of a Gaussian curve fit to the TPPL count histogram for the Sierpinski carpet, pseudo-random and periodic nanostructures at six excitation wavelengths. The total yield of TPPL intensity is also shown in Fig. 5.6(b), revealing a generally higher response for the Sierpinski carpet gold nanostructure. Note that despite the higher monomer count for the periodic nanostructure (729 vs 512 monomers), the Sierpinski carpet nanostructure has a higher total TPPL count. 
(a)


(b)
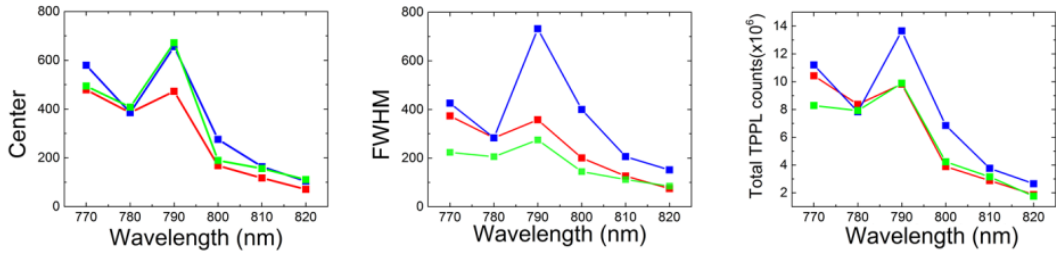

Fig. 5.6: (a) Histograms of TPPL counts of the Sierpinski carpet (blue), pseudo-random (red) and periodic (green) gold nanostructures at excitation wavelength of 770, 780, 790, 800, 810 and 820 $\mathrm{nm}$. (b) is the center and FWHM of Gaussian curve fitting of histogram, and total yields (from left to right) of TPPL counts of the Sierpinski carpet (blue), pseudo-random (red) and periodic (green) gold nanostructures.

To extract further quantitative measurements from TPPL images, we estimate the experimental TPPL intensity enhancement according to [72]:

$$
\alpha_{\text {exp }}=\sqrt{\frac{\left\langle T P P L_{\text {sample }}\right\rangle\left\langle P_{\text {film }}\right\rangle^{2} A_{\text {film }}}{\left\langle T P P L_{\text {film }}\right\rangle\left\langle P_{\text {sample }}\right\rangle^{2} A_{\text {sample }}}}
$$

where $\left\langle T P P L_{\text {sample }}\right\rangle$ and $\left\langle T P P L_{\text {film }}\right\rangle$ are the area-averaged signals from TPPL images of the gold nanostructure and a single crystalline gold film, respectively. Likewise, $\left\langle P_{\text {sample }}\right\rangle$ and $\left\langle P_{\text {film }}\right\rangle$ are the average powers of the excitation beams incident on the sample and the film, respectively. In our 
experiments, we used $\left\langle P_{\text {sample }}\right\rangle \sim 0.7 \mathrm{~mW}$ and $\left\langle P_{\text {film }}\right\rangle \sim 7 \mathrm{~mW}$. Note that in our experiments the TPPL signal in the gold film and three nanostructures shows the same quadratic relation with excitation power. $A_{\text {film }}$ and $A_{\text {sample }}$ are the effective areas of the gold film and the gold nanostructure sample for generating TPPL, which is determined by the spot size of focused excitation laser and the area of gold, respectively. For simplicity, we set $A_{\text {film }}=A_{\text {sample }}$, which means that the calculated TPPL enhancement factor $\alpha$ will be underestimated [56].

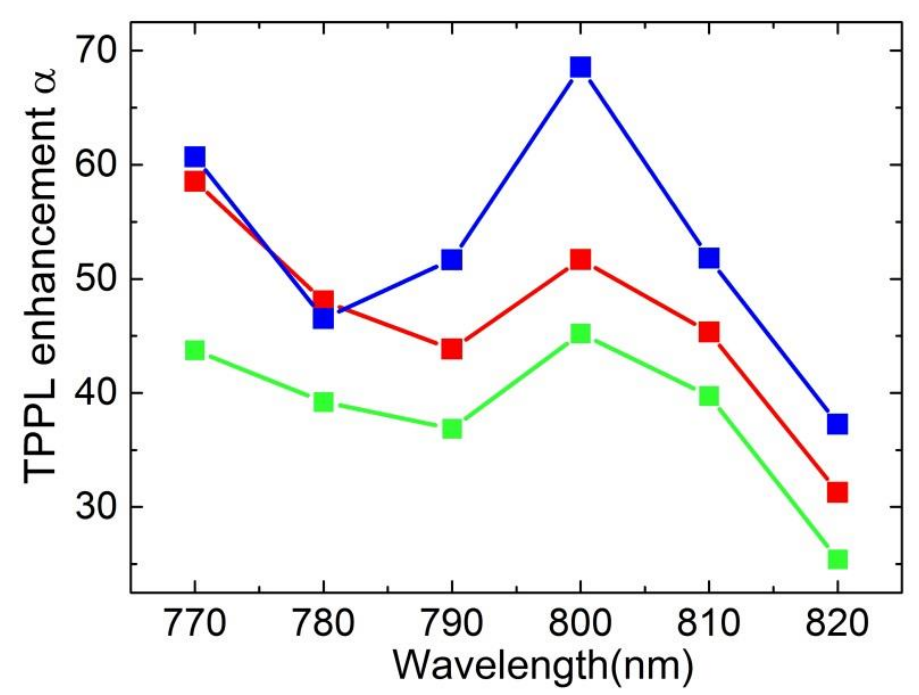

Fig. 5.7: TPPL enhancement factor $\alpha$ of the Sierpinski carpet (blue), pseudorandom (red) and periodic (green) gold nanostructures.

Fig. 5.7 shows the TPPL enhancement factor $\alpha$ calculated from Eq. (5.1). It features an interesting spectral behavior, falling off to low energy yet marked by a peak at $800 \mathrm{~nm}$ that is present in all three samples. At $800 \mathrm{~nm}$ the measured $\alpha_{\text {exp }}$ of the Sierpinski carpet nanostructure is highest, reaching $\sim 70$.

To determine whether the presence of hotspots has an influence on the enhancement factor, we recalculate $\alpha_{\text {exp }}$ following truncation of the high- 
intensity tails of the histograms. The effect is negligible: for the histogram of intensities from the Sierpinski carpet nanostructure obtained with excitation at $800 \mathrm{~nm}$ (Fig. 5.6(a)), truncating the tail at 900 TPPL counts yields an enhancement factor of 67, which is almost the same as the original value $\left(\alpha_{\text {exp }}\right.$ $=68$ ). For the periodic nanostructure, the enhancement factor upon truncation at 450 TPPL counts is identical to that found without truncation $\left(\alpha_{\text {exp }}=46\right)$. Likewise for the pseudo-random nanostructure, the enhancement factor is unchanged after truncating the tail at 350 TPPL counts in the histogram. Even if we truncate the tail of Sierpinski carpet gold nanostructure at 500 TPPL counts, i.e., well into the wing of the Gaussian curve, the value of $\alpha_{\text {exp }}$ is only reduced by $10 \%$. Hence the presence of hot spots does not influence dramatically the estimation of $\alpha_{\text {exp }}$.

Finally, to provide statistical significance to the observation of a higher enhancement factor $\alpha_{\exp }$ for the Sierpinski carpet nanostructure, we determined the TPPL yields and corresponding enhancement factors for subunits of the nanostructures. This analysis, based on the same data shown in the Fig. 5.5, was performed on two structures, the Sierpinski carpet and the periodic array, by dividing the TPPL images into four equal quadrants. Due to symmetry considerations, the values of $\alpha_{\text {exp }}$ are expected to be the same in each quadrant, hence any variations can be attributed to imperfections of sample fabrication such as inhomogeneity of the gap size and shape of monomers. We found that for both samples, the variation in $\alpha_{\text {exp }}$ for the four quadrants was less than $10 \%$ of the value found for the entire sample area (Fig. 5.7). This result confirms the higher near-field intensity enhancement for the Sierpinski carpet nanostructure.

\subsubsection{The FDTD simulation result of TPPL enhancement factor $\alpha$}

The TPPL intensity enhancement factor $\alpha$ gives us the average electric field intensity enhancement of gold nanostructures relative to a nonresonant gold film, and is calculated by [56]:

$$
\left|\alpha_{\text {sim }}\right|^{2}(\lambda)=\frac{\iint\left|E_{\text {sample }}(x, y, z, \lambda)\right|^{4} d x d y}{\iint\left|E_{\text {film }}(x, y, z, \lambda)\right|^{4} d x d y}
$$




\section{Chapter 5}

where the quantity $E_{\text {sample }}(x, y, z, \lambda)$ is the electric field on the surface of gold nanostructure samples, $E_{f i l m}(x, y, z, \lambda)$ is the electric field on the surface of a smooth gold film, and $\lambda$ is the wavelength of the incident light. When a plane wave is incident on the gold nanostructure, the electric field decays exponentially inside the gold nanostructure [56], therefore, in the calculation of the simulated TPPL intensity enhancement, we only need to consider the integral of $\left|E_{\text {sample }}(x, y, z, \lambda)\right|^{4}$ over the surface of a gold nanostructure and $\left|E_{\text {film }}(x, y, z, \lambda)\right|^{4}$ integrated over the same area of the total surface area of the gold nanostructure.

(a)

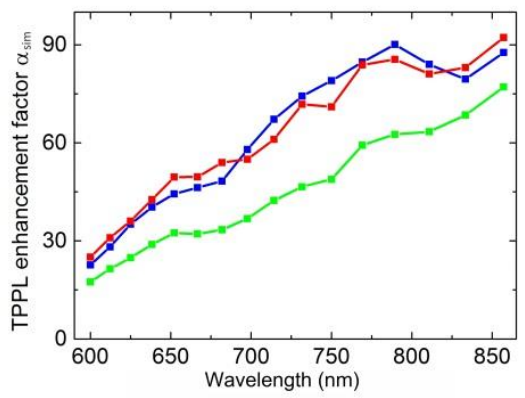

(b)

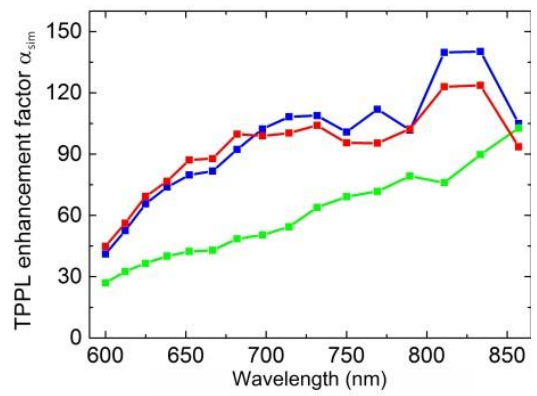

Fig. 5.8: (a), (b) show calculated TPPL enhancement factor $\alpha$ of the Sierpinski carpet (blue), pseudo-random (red) and periodic (green) gold nanostructures. The minimum mesh size for (a) is $7 \mathrm{~nm}$ and for (b) $5 \mathrm{~nm}$.

We used the FDTD method as in the calculation of scattering spectra to calculate the electric field on the surface of gold nanostructures and on a smooth gold film at different incident wavelengths. Fig. 5.8(a), (b) are simulated results of the TPPL enhancement factor $\alpha_{\text {sim }}$ for three nanostructures with the minimum mesh size 5 and $7 \mathrm{~nm}$, respectively. We can observe the poor convergence in the calculated TPPL field enhancement $\alpha_{\text {sim }}$. The trend that we observe in the measurements seems to be validated that the Sierpinski carpet and pseudo-random nanostructures have higher $\alpha_{\text {sim }}$ than that of the periodic one. However, the poor-convergence results due to the insufficient computer memory suggest that we need more efficient method to analyze and simulate the TPPL field enhancement $\alpha_{\text {sim }}$. 


\subsection{Conclusion}

We characterized the Sierpinski carpet gold nanostructure, fabricated from single crystalline gold flakes, demonstrating its potential to serve as a broadband optical antenna. Dark-field scattering microscopy showed that the Sierpinski carpet morphology behaved similar to an arbitrary-chosen pseudorandom gold nanostructure. Furthermore, TPPL measurements showed that the Sierpinski carpet nanostructure has a near-field enhancement factor of 70 upon excitation with $800 \mathrm{~nm}$ light, which was more than $50 \%$ higher than the other two geometries studied. We tentatively attribute this intensity enhancement to the long-range order present in the Sierpinski carpet optical antenna. Based on these results, the Sierpinski carpet morphology is clearly an interesting antenna design to achieve both broadband response and high near-field enhancement. We cannot exclude the possibility that other pseudorandom morphologies may perform even better, however, given its easy implementation we conclude that the self-similarity of the fractal geometry provides a simple design principle and can serve as an initial morphology for further optimization on light-harvesting applications, e.g., by the particle swarm optimization algorithm or an evolutionary strategy [73] [74]. 


\section{Chapter 6}

\section{Observation of surface lattice resonance}

In this chapter we will describe the observation of a surface lattice resonance of a Sierpinski carpet optical antenna by using back focal plane microscopy. We relate our findings with two equally valid views on the structure, one is relating it to the SLR in a periodic metal particle array, showing that the Fourier amplitude of the fractal optical antenna plays a similar role as the lattice vector in gratings and periodic arrays. Secondly, we relate the spectral feature in the scattering measurement with the dominant eigenmode found by using the eigen-decomposition method. The appearance of Fano line shapes in the scattering spectrum of the antenna is discussed. The coupled-dipole approximation and finite-difference time-domain (FDTD) numerical simulations are adopted to validate and understand our experimental findings. 


\section{Chapter 6}

Several previous studies have shown how resonant interactions of localized plasmons in periodic arrangements lead to narrow linewidth spectral features [41, 42, 43, 44, 40, 45]. The origin of these resonances was shown to be well described by a coupleddipole model in which the broad localized surface plasmon resonances of the particles are described as dipolar resonators [40]. An effective coupling between the particles leads to sharp spectral features near the condition for the (dis)appearance of a diffraction order, i.e. when the lattice vector $G=2 \pi / d$ crosses the light line $k=\omega / c$ with $\mathrm{d}$ the lattice spacing, $\omega$ the angular frequency and $\mathrm{c}$ the speed of light in the medium. To describe the collective resonance of the particles we use the often-employed term surface lattice resonance (SLR); note that in literature the terms Wood's- and Rayleigh anomaly are also often used in this context [75] [76] [77]. Translating the results from periodic lattices to non-periodic structures such as quasi-periodic or fractal structures is of particular interest as these configurations generate more densely-packed features in reciprocal space [78] [69] [79]. As a result, more isotropic behavior of diffracted light in terms of radiated directions or features that are less regularly spectrally spaced can be expected [80].

\subsection{Sample design}

(a)

(c)
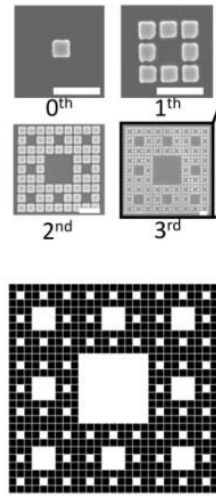

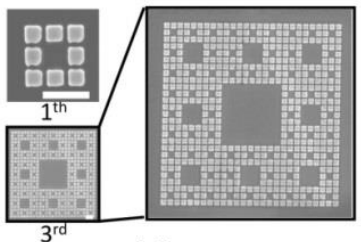

(d)

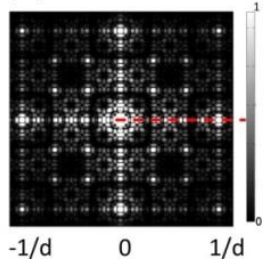

(b)

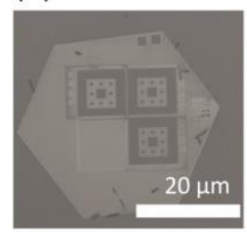

(e)

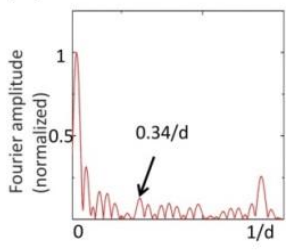

Fig. 6.1: (a) The Sierpinski carpet optical antennas fabricated on single crystalline gold flakes by focused ion beam milling. White scale bar is $500 \mathrm{~nm}$ for each sample. (b) shows three Sierpinski carpet optical antennas fabricated on a single-crystalline gold flake by FIB milling. (c) shows the schematic of the Sierpinski carpet optical antenna in our experiment. (d) shows the Fourier amplitude spectra of (c). $d$ is the minimum distance between monomers. (e) shows the line profile of the red dashed line in (c). 
Fig. 6.1(a) shows the $0^{\text {th }}-3^{\text {rd }}$ Sierpinski carpet optical antennas fabricated on single crystalline gold flakes by focused ion beam milling. The square monomer size is 200 (width) $\times 200$ (length) $\times 40$ (thickness) $\mathrm{nm}^{3}$. The gap distance between each monomer is $20 \mathrm{~nm}$. In this chapter we focus our discussion on the $3^{\text {rd }}$ order Sierpinski carpet optical antenna with 512 monomers. It is instructive to look at the Fourier transform of the idealized structure (Fig. 6.1(c)), as shown in Fig. 6.1(d). Many spots are found within the $1 / d$ "unit cell," spaced predominantly at $1 / 3$ of the inverse lattice spacing.

\subsection{The FDTD method and coupled dipole approximation}

Back focal plane microscopy is used to image the diffraction pattern of the optical antenna, formed by incident light at wavelengths $1.3-2.25 \mathrm{eV}$ (550 $950 \mathrm{~nm})$. The dipole moment $P=\left(P_{x}, P_{y}, P_{z}\right)$ of each monomer under the xpolarized plane wave excitation can be calculated by the coupled dipole approximation. The coupled dipole equation is introduced in Eq. (4.1). According to the fundamental principle of Fourier optics, the far-field electric field is entirely defined by the Fourier spectrum of the electric field in the object plane [23] [81]. Therefore, we calculate the Fourier spectrum of the dipole moment $P_{x}$ by Eq. (4.15). By the comparison of the Fourier amplitude spectrum of the dipole moment only with $P_{x}$ and with all three components $P_{x}$, $P_{y}$ and $P_{z}$, we may neglect components $P_{y}$ and $P_{z}$ here due to $\left|P_{x}\right| \gg\left|P_{y}\right|$, $\left|P_{z}\right|$ under a x-polarized plane wave excitation.

In addition to coupled dipole approximation, we also adopt the finitedifference time-domain (FDTD, Lumerical Solutions, Inc) numerical method to calculate the far field pattern of the Sierpinski carpet optical antenna at different incident wavelengths. The FDTD method includes the effect of glass substrate and multipolar interaction between monomers, whereas in the coupled dipole approximation we merely consider the dipolar interaction between each monomer and the effect of the glass substrate is not included. The monomer of sample is modeled as a gold rectangle with dimension of $200 \times 200 \times 40 \mathrm{~nm}^{3}$ (width $\times$ length $\times$ height) on the glass substrate. The mesh refinement is "comformal variant 1 " and the minimum mesh size is $4 \mathrm{~nm}$. The total-field scattered-field (TFSF) plane wave source with normal incidence on the gold nanostructures is used as excitation source. The dielectric constant of gold and glass substrate in the coupled dipole 
approximation and the FDTD method are from the experimental data [22] and [28], respectively.

\subsection{Comparison of diffraction images with calculated results}

Figs. 6.2(a) - (c) show the diffraction patterns observed on the back focal plane (BFP) of the back focal plane microscope. These images show the light scattered by the optical antenna upon illumination with light of incident photon energy 1.9, 1.65 and $1.46 \mathrm{eV}(650,750$ and $850 \mathrm{~nm})$, respectively. The red circles indicate the wave vector of the light line in air. The measured diffraction patterns show a strong resemblance to the calculated Fourier amplitude of the Sierpinski carpet geometry (shown in Fig. 6.1(d)). As the incident wavelength increases (photon energy decreases), the diffraction pattern gradually extends beyond the observable region of the back focal plane microscopy. We note that the diffraction patterns also include spots corresponding to scattered light with wave vectors larger than that of the incident light k0, i.e. beyond the red circles in each panel of Fig. 6.2. These wave vectors actually correspond to evanescent waves in air, which can be visualized in the BFP image thanks to the 1.4 N.A. oil immersion objective in our back focal plane microscopy.

The most striking feature in the BFP images of Fig. 6.2(b) is the appearance of two intense spots at the incident photon energy of $1.65 \mathrm{eV}$ marked $\mathrm{a}$ and $\mathrm{b}$. The diffraction signal in this region of k-space extending beyond the light line in air is much weaker when incident light of 1.9 and $1.46 \mathrm{eV}$ is used, suggesting a significant spectral dependence.

We assign the high intensity diffraction signals $a$ and $b$ to a surface lattice resonance. The individual particles within the Sierpinski carpet structure exhibit a resonant interaction that occurs at a photon energy just after the disappearance of a diffraction maximum of the fractal leading to a high intensity surface wave on the air side. Energy in this excited mode can leak out into the glass substrate and can be detected in the BFP, in the same way that propagating surface plasmons on metal films can be observed using leakage radiation microscopy [82]. 


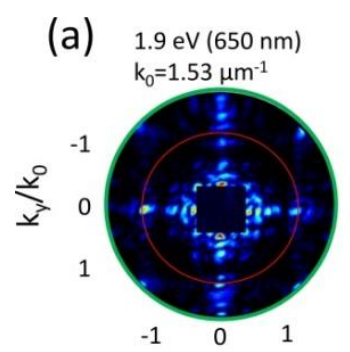

(d)

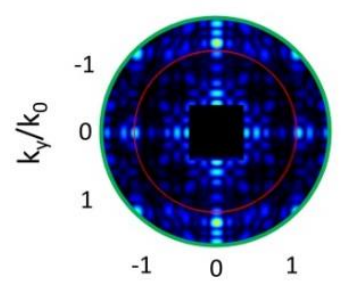

(g)

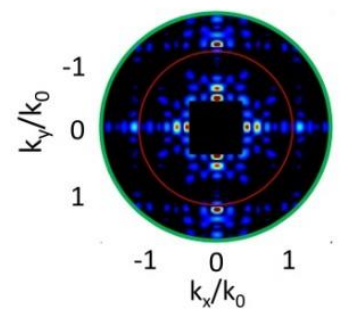

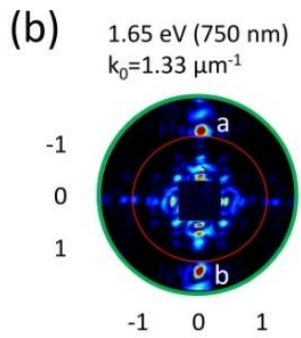

(e)

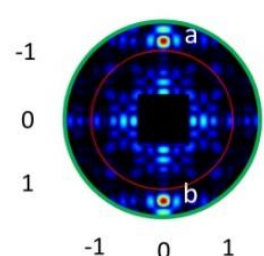

(h)

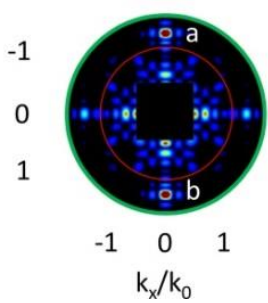

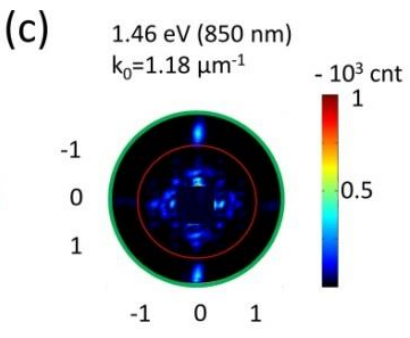

(f)

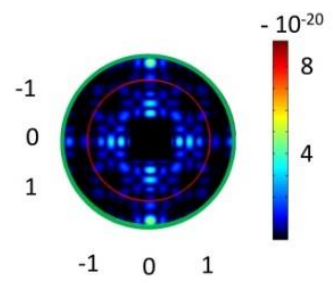

(i)

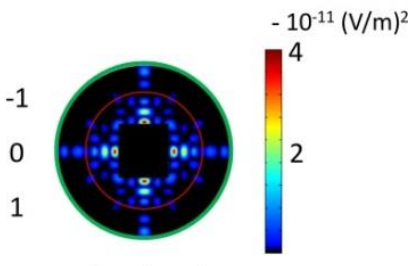

$\begin{array}{lll}-1 & 0 & 1 \\ k_{\mathrm{x}} / k_{0} & \end{array}$

Fig. 6.2: Panels (a) - (c) show the BFP images of the Sierpinski carpet optical antenna with the incident light photon energy at $1.9,1.65$ and $1.46 \mathrm{eV}$, respectively. The polarizer and analyzer in the back focal plane microscopy are both set in the x direction. The CCD count of (a) - (c) is normalized with the incident light power, exposure time and quantum efficiency of CCD. Note that the central region, where the $0^{\text {th }}$ order diffracted light would appear in the image, is blocked by the beam blocker in the back focal plane microscopy. Panels (d) - (f) show the Fourier amplitude spectra of $P_{x}$ of optical antenna calculated by the coupled dipole approximation with the x-polarized incident light at photon energy 1.9, 1.65 and $1.46 \mathrm{eV}$. Likewise, Panels (g) - (i) show the far field radiation pattern calculated by the FDTD method with the far field transformation of the electric field at the plane of $10 \mathrm{~nm}$ below the interface between the sample and glass substrate. Note that the central regions of (d) - (f) and (g) - (i), corresponding to the $0^{\text {th }}$ order diffracted light in BFP images, are set to zero for a better comparison with the BFP images (a) - (c). The red and green circles in each picture correspond to the wave vector of the light line in air and in the glass substrate, respectively. The $k_{0}$ for three photon energies are set to the same scale, resulting in different sized circles in each column. 
We can illustrate this feature by examining the Fourier amplitude spectra of $P_{x}$ calculated by the coupled dipole approximation in panels (d) (f): the diffraction spots appearing between the red and green circles (representing the wave vector of incident light in air and in the glass substrate, respectively) have the transverse wave vector $k_{\|}$satisfying the condition: $k_{0}<k_{\|}<k_{g}$, where $k_{g}$ is the wave vector of incident light in the glass substrate, i.e., $k_{g}=n k_{0}, \mathrm{n}$ is the refractive index of the glass substrate 1.48. If we let $\left(k_{x}, k_{y}, k_{z}\right)$ represent the wave vector of diffracted light, then by momentum conservation we have $\hbar \vec{k}_{0}=\hbar\left(\vec{k}_{\|}+k_{z} \hat{z}\right)$ for the diffracted light in the air and $\hbar \vec{k}_{g}=\hbar\left(\vec{k}_{\|}+k_{z} \hat{z}\right)$ in the glass substrate, where $\vec{k}_{\|}=\left(k_{x}, k_{y}\right)$ and $\hat{z}$ is the unit vector in $\mathrm{z}$ direction. We have $k_{z}=i\left(k_{\|}^{2}-k_{0}^{2}\right)^{1 / 2}$ for the diffracted light in the air, while $k_{z}=\left(k_{g}^{2}-k_{\|}^{2}\right)^{1 / 2}$ for the diffracted light in the glass substrate. Since $k_{z}$ is imaginary in the air but real in the glass substrate, the diffraction spot located between red and green circles corresponds to light that is evanescent in air but propagative in the glass substrate, making it visible in our images. Note that the images in panels (d) - (f) are not the far field radiation pattern; instead, they provide a picture of the regions of wave vectors in the Fourier k-space that are excited by the incident plane wave.

In addition, the far-field radiation patterns emerging after light with photon energy $1.9,1.65$ and $1.46 \mathrm{eV}$ is incident on our Sierpinski carpet optical antenna are shown in the bottom row of the Fig. 6.2, in panels (g) - (i). These patterns are retrieved from the far field transformation of the electric field present in the plane $10 \mathrm{~nm}$ below the interface between the sample and glass substrate calculated by the FDTD method (Lumerical Solutions, Inc.), which is shown in Fig. 6.3(a) - (f). Examining the panels in the middle and bottom rows of Fig. 6.2, we can see that they are both similar to the Fourier amplitude of the Sierpinski carpet geometry (see Fig. 6.1(d)) and the measured diffraction patterns in the top row, (a) - (c).

We also calculate the electric field at the plane of $10 \mathrm{~nm}$ above the sample (in the air) with incident wavelength at $1.9,1.65$ and $1.46 \mathrm{eV}$ respectively as shown in Figs. 6.3(g) - (i). (j) - (l) are the far field projection of the electric fields shown in (g) - (i), respectively. Note that (a) - (c) and (g) - (i) show the near-field contributions of the electric field; their far-fields projections are quite different. The far-field projections of the electric field in 
the glass (panels (d) - (f)) contains some spots which disappear in panels (j) (l) corresponding to the evanescent waves in the air.
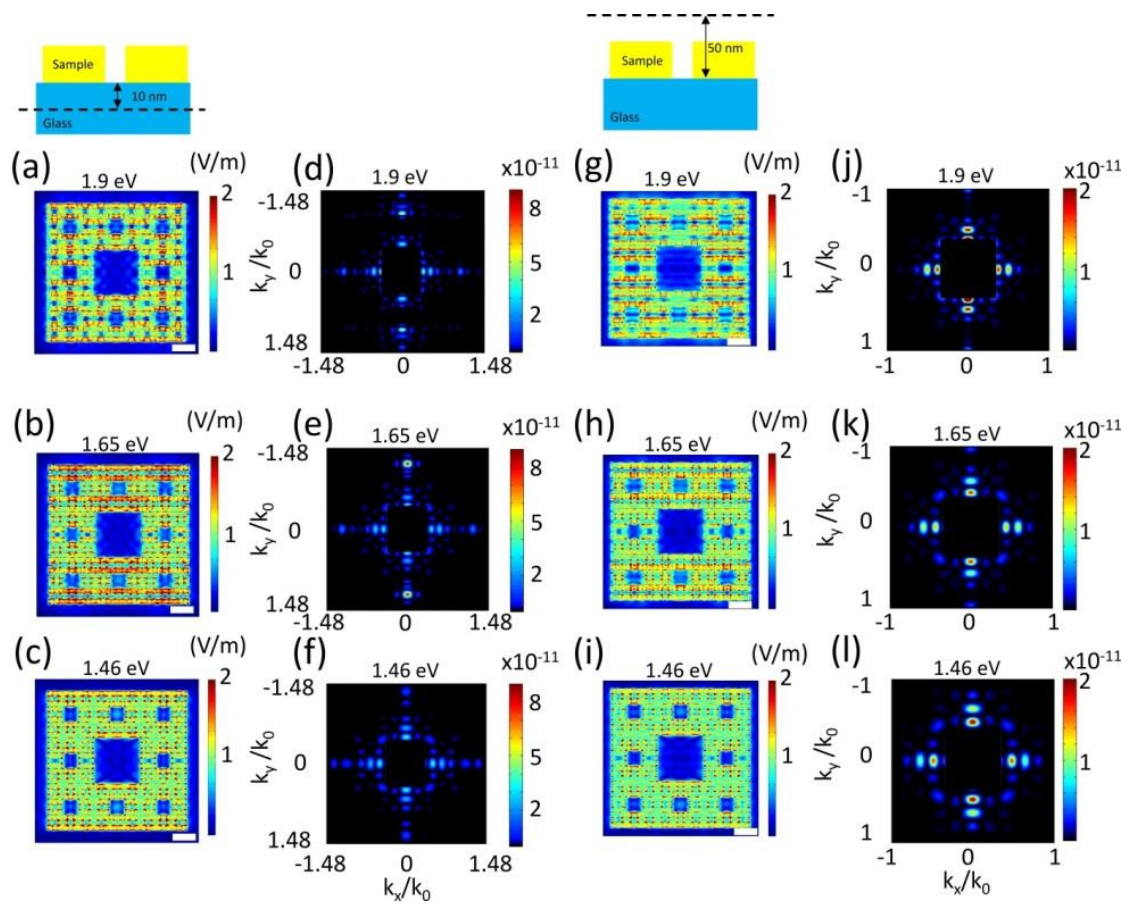

Fig. 6.3: (a) - (c) are the electric field at the plane of $10 \mathrm{~nm}$ below the interface between the sample and glass substrate with the incident light wavelength at $1.9,1.65$ and $1.46 \mathrm{eV}$. (d) - (f) are the far field projection of electric fields (a) - (c), respectively. (g) - (i) are the electric field at the plane of $50 \mathrm{~nm}$ above the interface with the incident light wavelength at 1.9, 1.65 and 1.46 eV. (j) - (1) are the far field projection of electric fields (g) - (i), respectively. The unit of the color bar for (a) - (c) and (g) - (i) is V/m and $(\mathrm{V} / \mathrm{m})^{2}$ for (d) - (f) and (j) - (l). Note that the region of $0^{\text {th }}$ order diffracted light in (d) - (f) and (j) - (l) is set to zero for a better comparison with the BFP images in Fig.6.2(a) - (c). 


\subsection{The dispersion relation from BFP images}

(a)

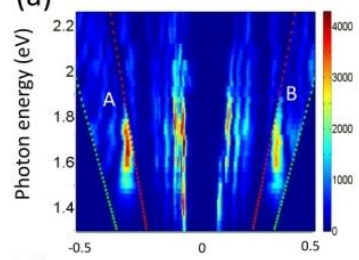

(d)

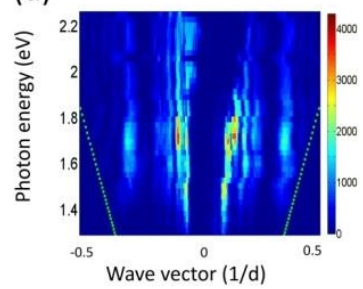

(b)

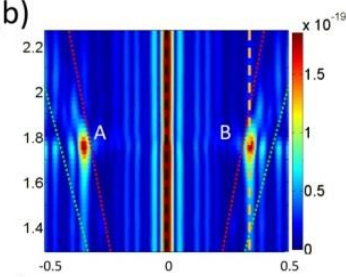

(e)

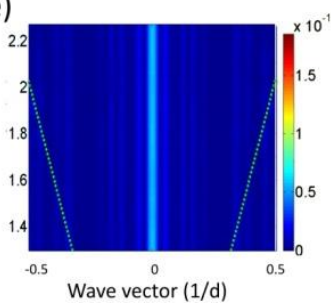

(c)

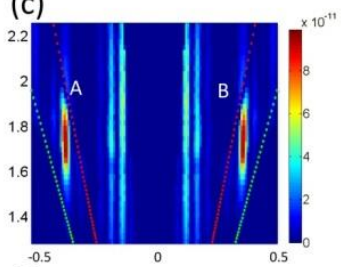

(f)

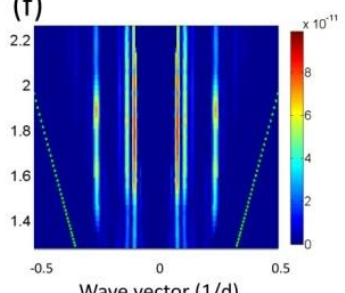

Fig. 6.4: (a) is the line profiles at the direction of $\left(k_{x}, k_{y}\right)=(0,1)$ of measured BFP images at incident photon energy $1.3-2.25 \mathrm{eV}$. Similarly, (b) and (c) are the line profiles of the Fourier amplitude spectra of $P_{x}$ calculated by the coupled dipole approximation and far field radiation pattern calculated by the FDTD method at incident wavelengths $1.3-2.25 \mathrm{eV}$, respectively. The red and green dot lines in each picture represent the wave vector of light line in air and glass substrate, respectively. $d$ is the minimum distance between monomers, here $d=220 \mathrm{~nm}$.

The spectral dependence of intense spots $a$ and $b$ in Fig. 6.2 is elaborated by plots of line profiles in the direction of $\left(k_{x}, k_{y}\right)=(0,1)$ for the diffraction pattern of the Sierpinski carpet optical antenna measured with incident light at photon energy ranging from $1.3-2.25 \mathrm{eV}$. Fig. 6.4(a) can be regarded as the dispersion relation $\omega-\mathrm{k}$ at the direction of reciprocal vectors $\left(k_{x}, k_{y}\right)=(0$, 1), which also means that Fig. 6.4(a) shows a standing wave patterns with large amplitude in regions A, B. Analogous to this representation of the measured data, Figs.6.4(b) and (c) show line profiles of the Fourier amplitude spectra of $P_{x}$ calculated by the coupled dipole approximation and the far field radiation patterns calculated by FDTD method, respectively.

All three panels of Fig. 6.4 show the same distinctive features: vertical lines and bright regions marked by intense signals A and B. The vertical lines arise from the non-periodic analogue of the familiar diffraction grating formula: the parallel wave vector component of the diffracted light $\mathrm{k}_{\|}$is a 
multiple of the discrete lattice vector $G$, which is independent on wavelength $\left(k_{\|}=n G\right)$. In the finite and non-periodic fractal structure $G$ is replaced by the amplitude of the Fourier transform of the structure, therefore vertical lines appear, corresponding to the high amplitude Fourier components in the Sierpinski carpet optical antenna.

The intense signals A and B lie along the edge of the light line in air (red dotted line), suggests that they arise from the evanescent wave in air. These evanescent waves are also responsible for the distortion and overlap between the vertical lines in the vicinity of enhancement regions. This distortion along the line at around $1.8 \mathrm{eV}$ is clearly seen in the Fig. 6.4(b), and is explained by the presence of a Fano resonance, which will be explained later.

To further support the assignment of signals A and B as arising from evanescent waves, we repeat the measurements upon immersing the optical antenna in refractive-index matching oil. Figs. 6.4(d) - (f) are the measured and calculated dispersion relation when the sample is immersed in the refractive index-matching oil. The effect is markedly obvious: the signals completely disappear, both in measurements and simulations.

The FDTD method allows additional evidence that signals $\mathrm{A}$ and $\mathrm{B}$ correspond to evanescent waves: in the Fig. 6.3, we find the pattern of the electric field within the glass substrate indeed contains the intensive spots $a_{3}$ and $b_{3}$ as indicated in the Fig. 6.2(h), whereas the pattern of the electric field above (in air) does not. 


\subsection{The calculation of dominate eigenmode by eigen-decomposition method}

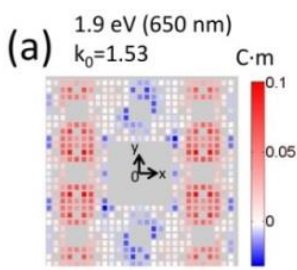

(d)

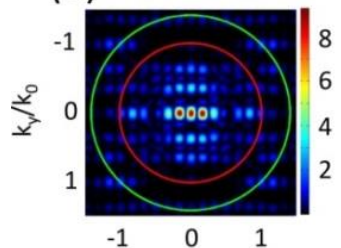

(g)

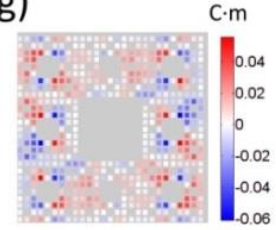

(J)

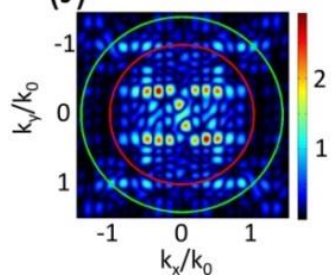

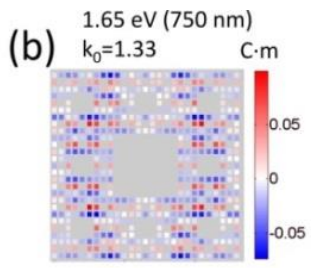

(e)

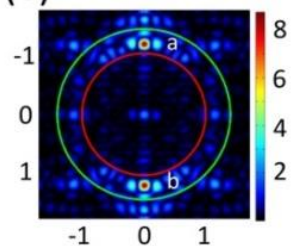

(h)

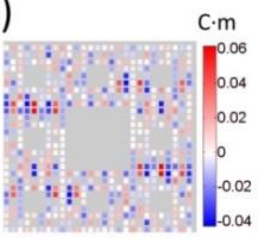

(k)

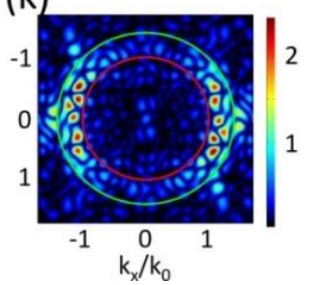

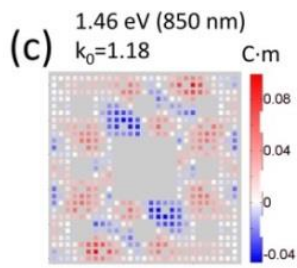

(f)

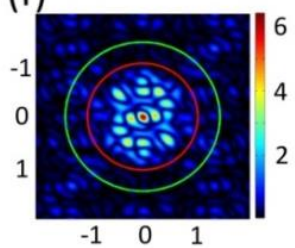

(i) $C \cdot m$

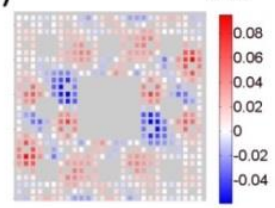

(I)

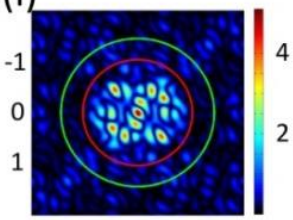

-1 $\underset{k_{x} / k_{0}}{0}{ }^{1}$

Fig. 6.5: Panels (a) - (c) represent the real part of $P_{x}$ of the dominant eigenmode excited with incident light at 1.9, 1.65 and $1.46 \mathrm{eV}$. Panels (d) - (f) show the calculated Fourier amplitude spectra of $P_{x}$ in (a) - (c), respectively, where the red and green circles represent wave vector of the light line in air and in the glass substrate, respectively. (g) - (i) are the real part of $P_{y}$ of the dominate eigenmode at excitation wavelength 1.9, 1.65 and $1.46 \mathrm{eV}$. (j) - (1) show the Fourier spectra of $P_{y}$ at $1.9,1.65$ and $1.46 \mathrm{eV}$. In (j) - (1), the red and green circle represent wave vector of light line in the air and glass substrate, respectively. $\mathrm{k}_{0}$ is the wave vector of incident light in the air. 
To determine the origin of the signals A, B in Fig. 6.4, we extract the dominant eigenmode of the optical antenna by the eigen-decomposition method [60] [61]. The dominant eigenmode is defined as the eigenmode with a heaviest weight among all eigenmodes. We diagonalize the interaction matrix $A_{m, n}$ in the coupled dipole approximation to get the $1536(512 \times 3)$ eigenvectors $\left|P_{i}\right\rangle, \mathrm{i}=1,2, \ldots, 1536$. The dipole moment $\vec{P}$ of the optical antenna excited by a x-polarized plane wave can be written as the linear combination of eigenvectors $\left|P_{i}\right\rangle:|P\rangle=\sum_{i} c_{i}\left|P_{i}\right\rangle$, where $\mid P>$ is the complex column vector of dipole moment and $c_{\mathrm{i}}$ is the complex coefficient. The dominant eigenmode for $\mid P>$ is defined as the individual eigenmode $\mid P_{i}>$ having maximum $\left|\mathrm{c}_{\mathrm{i}}\right|$ among all eigenmodes. To obtain the coefficient $c_{i}$, we multiply $\left\langle P_{j}\right|$ with $\mid P>:\left\langle P_{j}\right| P>=\sum_{i} c_{i}\left\langle P_{j}\right| P_{i}>$. The indices $i, j$ $=1,2, \ldots, 1536$. Note that $\left\langle P_{j} \mid P_{i}\right\rangle \neq \delta_{i j}$, where $\delta_{i j}=1$ for $i=j, \delta_{i j}=0$ for $i \neq j$, because eigenvectors $\left|P_{i}\right\rangle, i=1,2, \ldots, 1536$, are not an orthogonal set [83]. Therefore, we solve 1536 linear equations to determine $c_{i}$. Figs. 6.5(a) - (c) and (d) - (f) show the dipole moment $P_{X}$ and their corresponding Fourier amplitude of the dominant eigenmode of optical antenna at excitation photon energy $1.9,1.65$ and $1.46 \mathrm{eV}$, respectively. We find, at $1.65 \mathrm{eV}$, most of the wave vectors are located outside the light line of the incident light in air (the red circle) as shown in Fig. 6.5(e), indicating its dark (subradiance) property. On the contrary, the dominant eigenmodes at 1.9 and $1.46 \mathrm{eV}$ are bright (superradiant), shown in Figs. 6.5(d) and (f), respectively. We can easily recognize that the intense spots $\mathrm{a}, \mathrm{b}$ in Fig. 6.5(e) correspond to a, b in Figs. 6.2(b), (h). Therefore, we conclude that the signals A and B in the Fig. 3 arise from the resonance of the dark eigenmode shown in Fig. 6.4(b). Fig. 6.6 shows $\left|c_{i}\right|$ for each eigenmodes at $1.9,1.65$ and $1.46 \mathrm{eV}$, in which $\left|c_{i}\right|$ are sorted from maximum to minimum.

We find the condition analogous to a surface lattice resonance in a periodic grating provides the mechanism for the resonance of a subradiant eigenmode. The resonance we observe in Fig. 6.2(b) is triggered by the inplane diffracted light. This also means the in-plane diffracted wave has a periodicity that matches with one of the fundamental periodicities of our optical antenna. From Fig. 6.5(b) it can be found that the periodicity matches the length of 3 monomers, which fits well to the occurrence of the leakage radiation of the LSPR at $0.34 / d$ (indicated with the arrow in Fig. 6.1(e)). 

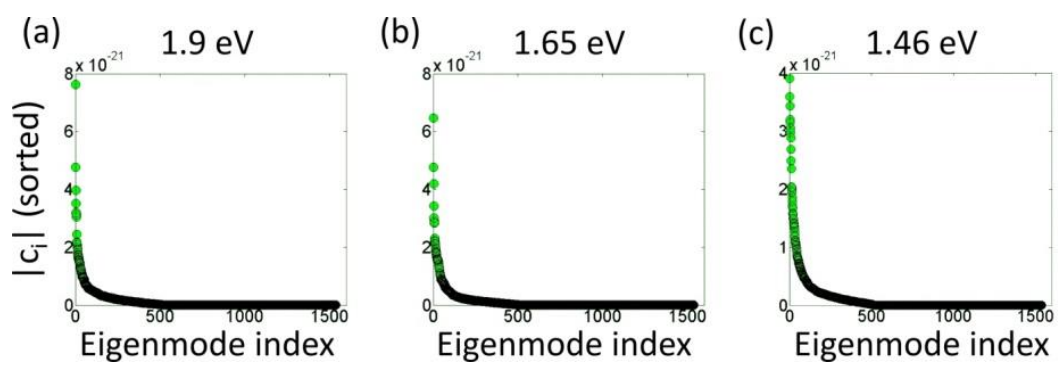

Fig. 6.6: The sorted $\left|c_{i}\right|$ of each eigenmode of the Sierpinski carpet optical antenna for (a) 1.9 (b) 1.65 (c) $1.46 \mathrm{eV}$. For (a), (c) the dominant eigenmodes (the eigenmode having maximum $\left|c_{i}\right|$ ) are bright; as for $1.65 \mathrm{eV}$, it is dark, which was just shown in Fig. 6.5.

\subsection{The Fano-dip in the scattering spectrum}

An important characteristic of a subradiant eigenmode such as an SLR is that it can generate a Fano profile in the extinction or scattering spectrum of plasmonic nanostructures [39] [84] [85]. In general, Fano profiles arise from the destructive interference between a resonant mode and a continuum. In plasmonic structures this can arise in different forms, for instance in (1) the interference between a dark and a bright eigenmode or (2) the interference between a plasmonic resonance and the directly transmitted light. Unfortunately the latter is difficult to measure in a relatively small structure as described here because the scattering is of much lower magnitude than the direct transmission. We therefore aimed to determine if a Fano-like interference between dark and bright modes can be detected in scattering, despite the fact that more than 1500 eigenmodes are present in the structure.

Fig. 6.7(a) shows the measured scattering spectrum of the Sierpinski carpet optical antenna, obtained by a white-light dark field microscopy introduced in Chapter 3 [1]. The annular incidence polar angle of white light ranges from $20^{\circ}-50^{\circ}$. The scattering spectrum features a significant dip around $730 \mathrm{~nm}$, which coincides with the spectral region where the intense signals A, B appear in the line profiles of the BFP images shown in the Fig. 6.4(a). We use a shaded region in the Fig. 6.7(a) to show this correspondence. 
(a)

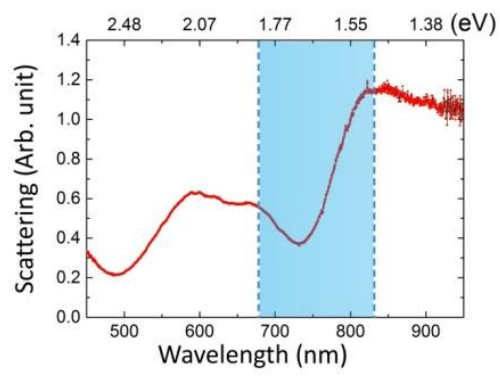

(b)

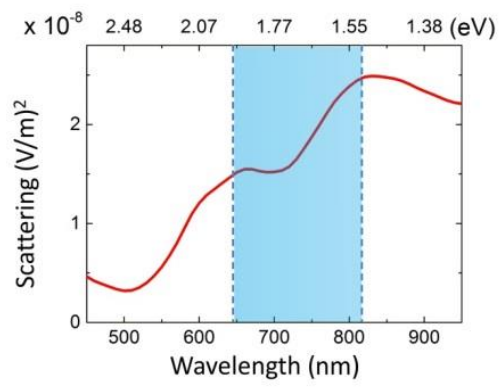

(c)

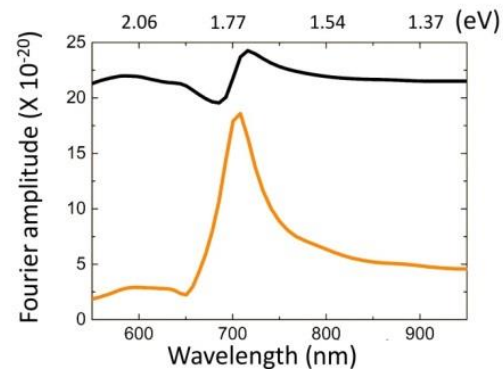

Fig. 6.7: Panel (a) shows the measured scattering spectrum of the Sierpinski carpet optical antenna with $x$-polarized incident light. The grey shaded zone corresponds to the region of intense signal A, B in Fig. 6.4(a). Panel (b) shows the scattering cross section calculated by the FDTD method and the grey shaded region corresponds to A, B in Fig. 6.4(c). The black curve in the Panel (d) is the line profile of the black dash line in Fig. 6.4(b), while the orange curve is the line profile of the orange dash line in Fig. 6.4.(b). 
The FDTD method is adopted to calculate the far-field scattering power; the results are presented in Fig. 6.7(b). The shaded region again corresponds to the regions A, B in Fig. 6.4(c). The scattering spectrum of Fig. 6.7(b) indicates a dip around $720 \mathrm{~nm}$, which is close to the measured result in the Fig. 6.7(a). Numerical results were obtained using finite difference time domain (FDTD, Lumerical Solutions Inc.) calculations. The parameter settings of FDTD method are the same as the calculation for the diffraction patterns of the Sierpinski carpet optical antenna. To simulate the experiment conditions, we use a reversed configuration shown in the Fig. 6.8(a): the white light incident normally on the sample from the air side, and the collection polar angle $\theta$ of the scattered light is set to be $20^{\circ}$ to $50^{\circ}$ in the side of glass substrate. We calculate the near-field electric field at the plane of 10 $\mathrm{nm}$ below the interface between the sample and glass substrate, then we use the "near to far field projections" in the FDTD method to get the far field radiation pattern. From the far field radiation pattern at different wavelengths, we can sum the total far-field radiation power within the collection angle $\theta=$ $20^{\circ}-50^{\circ}$ at each wavelengths to get the scattering spectrum. Fig. 6.8(b) shows an example of the far field radiation pattern at the photon energy 1.77 $\mathrm{eV}$. The range of collection angle $\theta$ is $20^{\circ}-50^{\circ}$.

To determine if the drop in scattering intensity around $730 \mathrm{~nm}$ (Fig. 6.7(a)) is a Fano-dip caused by the SLR, we invoke the coupled dipole approximation to provide a clear demonstration and explanation. Fig. 6.7(c) shows the line profiles of the black and orange dashed lines drawn in Fig. 6.4(b). We find that both line profiles exhibit the characteristic asymmetry of the Fano line-shape. In the black curve, the dip at around $690 \mathrm{~nm}$ and peak at around $710 \mathrm{~nm}$ arise from the $\pi$ phase jump of the dark eigenmode, which renders the destructive and constructive interference with other bright eigemodes, respectively. From the orange curve, we know the resonant wavelength of dark eigenmode is around $700 \mathrm{~nm}$. The Fano-dip at $690 \mathrm{~nm}$ of the orange curve can be explained by the destructive interference of the overlapped wave vectors between the dark eigenmode and other bright eigenmode within the red circle of Fig. 6.5(e). As the dark eigenmode resonates, its coefficient $c_{i}$ (shown in Fig. 6.6) increases to be maximum among all eigenmodes, which cancels the overlapped wave vectors of other bright eigenmodes within the red circle and consequently creates a dip in farfield scattering spectrum. The overlapped wave vectors of dark and bright eigenmodes within the red circle is equivalent to the overlapped far-field radiation pattern of the dark and bright eigenmode. This agrees with the 
finding of Frimmer et al. [86], that the Fano-dip requires an excellent overlap of the far-field radiation pattern of dark and bright eigenmode.

We believe the same type of interference between modes is observed in the experiments here, however, higher-order multipolar interactions and the leakage of the mode into the glass substrate lead to overall broadening and deviations from the simplified result obtained from the coupled dipole approximation. Also due to the complex mode structure of the fractal antenna and broad spectral features of plasmonics in the visible, it is challenging to show exactly which or how many modes are needed to generate the scattering spectrum of the fractal structure.

(a)

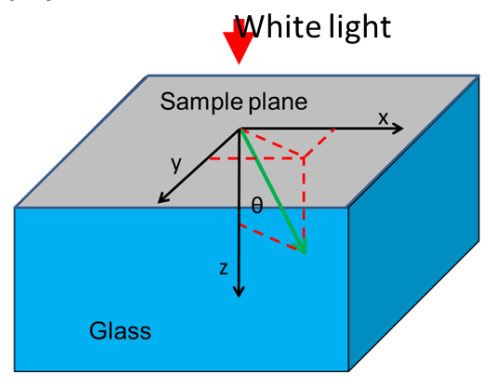

(b)

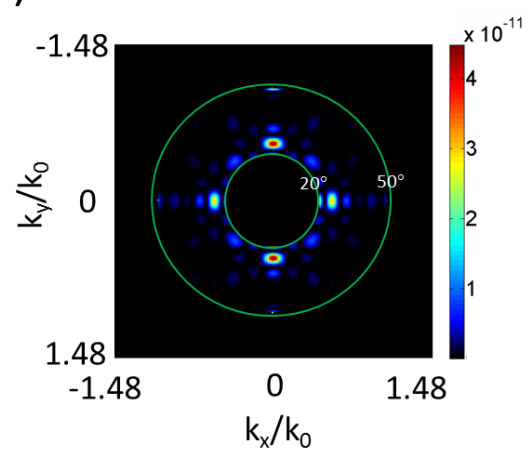

Fig. 6.8: (a) The reversed configuration in the FDTD calculation of the scattering spectrum. (b) The far field intensity at $1.77 \mathrm{eV}$. The range of polar angle $\theta$ between $20^{\circ}-50^{\circ}$ is the collection angle used in the FDTD calculation of the scattering spectrum. 


\subsubsection{Results and discussions}

(a)

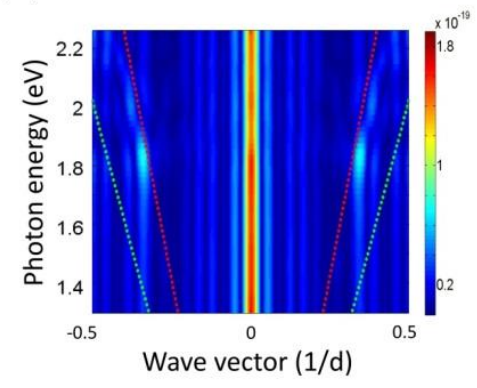

(b)

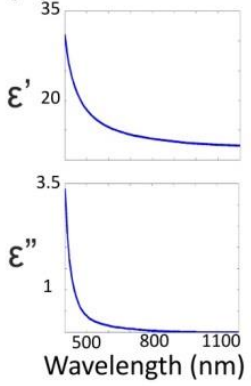

(c)

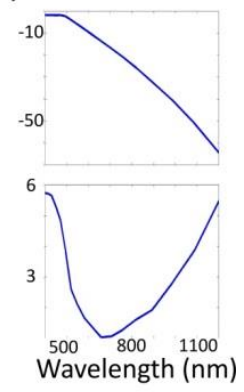

Fig. 6.9: (a) shows the dispersion relation similar to Fig. 6.4(b) but the material of monomers in the Sierpinski carpet optical antenna is silicon. The red and green dot lines in (a) and (b) represent the wave vector of light line in the air and glass substrate, respectively. (b) shows the dielectric constant of silicon $\varepsilon=\varepsilon^{\prime}+i \varepsilon^{\prime \prime}$ [28] used in the coupled dipole approximation calculation. (c) shows the dielectric constant of gold $\varepsilon=\varepsilon^{\prime}+i \varepsilon^{\prime \prime}$ [22] used in the coupled dipole approximation and FDTD numerical calculations .

We should note that the SLR we find here is not only limited to plasmonic materials. In fact, this SLR is an inherent property of the fractal geometry, and still exists, for example, if the material used in the coupled dipole approximation is changed from gold to silicon. Fig. 6.9(a) shows the dispersion relation similar to Fig. 6.4(b) but with the silicon as the material for the monomers in our optical antenna. Compared with the dispersion relation for the gold in Fig. 6.4(b), we find the presence of the intense spots corresponding to the SLR for silicon to be weaker. The higher signal intensity for gold comes from the coupling between the LSPR of monomers and the in-plane diffactive waves just like the case for periodic plasmonic arrays [87] [40].

The existence of a SLR in a fractal arrangement of plasmonic resonators is clearly demonstrated; however, several intriguing side issues remain. Firstly the SLR occurs when the light-line crosses the 0.34/d maximum in Fourier amplitude of the fractal structure. Though the amplitude of this component is certainly among the stronger peaks in the Fourier spectrum, it is unclear why conditions for a SLR are not met at other crossings. A 
possible solution of this lies in a detailed study of the eigenmodes that are extracted based on the CDA, though the large number of modes makes this a very complex puzzle. We tentatively attribute the SLR to the fractal arrangement of our Sierpinski carpet optical antenna, which renders an efficient subradiant eigenmode with a high eigenpolarizability [86]. Closely related, the second issue is how such a clear Fano resonance emerges from the coupled dipole approximation whereas a large number of modes is collectively responsible for the response of the structure.

\subsection{Conclusion}

In conclusion, we demonstrate a strong SLR of the Sierpinski carpet optical antenna, which is triggered by the mechanism analogous to the resonant Wood's anomaly for a periodic grating, can be visualized by a back focal plane microscopy. These findings can be applied to other fractal or aperiodic optical antennas, such as quasicrystalline, Fibonacci, Thue-Morse or checkerboard plasmonic arrays, etc. [69], to visualize the SLR in them. The quantification of the light energy stored in the near field of aperiodic or fractal plasmonic arrays by SLR can be retrieved after careful calibrations of $\mathrm{CCD}$ and objectives in back focal plane microscopy. Our findings will shed new insights on the design of nanophotonic devices [88] and plasmonic sensors [89] which exploit aperiodic or fractal plasmonic arrays. 


\section{Chapter 7}

\section{Outlook and applications}

Based on the experimental findings and theoretical works addressed in this thesis, we propose potential directions for future research and possible applications. 
In previous chapters we have investigated the optical properties of Sierpinski carpet optical antennas. In Chapter 4, the eigen-decomposition method has been adopted to analyze the property of eigenmodes. In Chapter 5, in addition to the Sierpinski carpet geometry we further consider two different morphologies - pseudo-random and periodic array of gold particles. We investigated their scattering spectra and near-field intensity enhancement. In Chapter 6, we visualized plasmonic modes of a Sierpinski carpet optical antenna on the Fourier plane of a back focal plane microscopy. A SLR of the Sierpinski carpet optical antenna is revealed and a Fano-dip caused by the SLR appears in the scattering spectrum. Our experimental techniques and theoretical methods can be generalized to other similar plasmonic nanostructures, and the application of Sierpinski carpet optical antennas can be useful to some areas requiring the enhancement of light-matter interaction. Below we will discuss some possible further investigations and applications that are related to previous works in this thesis.

\subsection{The investigations of other aperiodic or fractal metal nanoparticle arrays}

Aperiodic deterministic nanostructures provide the potential for the manipulation of localized electromagnetic fields and light scattering phenomena on planar optical chips [69] [68]. Periodic arrays support extensive Bloch waves, while random arrays sustain localized eigenmodes. However, random structures are irreproducible and lack predictive models and specific design rules for the optimization. These problems can be solved by the aperiodic arrays generated by deterministic mathematical rules.

There are many different types of aperiodic arrays which have been proposed and investigated such as quasicrystalline arrays [90] [91] [92], Fibonacci arrays [70] [93], Thue-Morse arrays and Rudin-Shapiro arrays [94], etc. Among all of these aperiodic arrays, the quasicrystalline arrays has drawn most of attentions.

In 1982, Shechtman discovered a quasicrystal with 10-fold symmetry in the diffraction pattern after he has rapidly chilled the molten mixture of aluminum and manganese [95]. The first paper about this discovery was then published in 1984 [96]. Since then, more than 100 different types of quasicrystals have been synthesized in the laboratory under carefully 
controlled conditions. The original theory suggested that quasicrystals can be as robust and stable as crystals, perhaps even forming under natural conditions [97].

Similar to the Sierpinski carpet, quasicrystals are neither periodic nor random. Quasicrystals show a long-range orientational order as well as a long-range quasiperiodic translational order [98]. However, no periodic translational order can be observed. Although they do not show any lattice periodicity, such structures exhibit a Fourier transform with essentially discrete Fourier peaks which is due to the long-range order. Fig. 7.1 shows the diffraction pattern of a quasicrystal of 10-fold symmetry, in which circles formed by ten bright spots can be found.

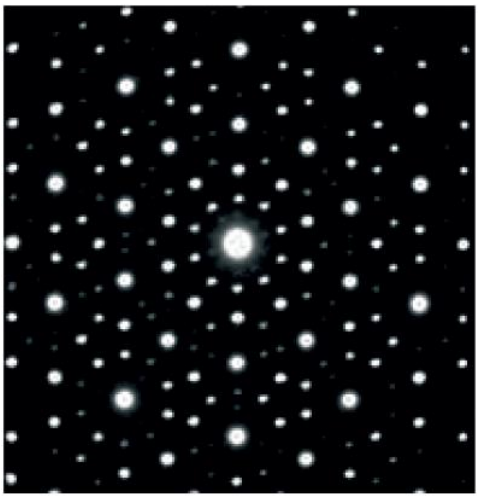

Fig. 7.1: 10-fold symmetry of a quasicrystal in the diffraction pattern [95].

The localization and eigenpolarizability spectra of eigenmodes of twodimensional quasicrystals consisting of metal nanoparticles has been investigated by the eigen-decomposition method [99] [100]. The quasicrystalline plasmonic arrays are also used to enhance the efficiency of solar cells [101]. Recently the plasmonic mode of a quasicrystal-the Penrose pattern made by silver nanoparticles has been studied by a Fourier microscopy [102], allowing the direct measurement of the intensity of various plasmonic modes. However in general, these studies lack discussion of tracking a specific subradiant eigenmode which exhibits strong surface 
lattice resonance (SLR) in plasmonic quasicrystals. We expect that the rotational symmetry and the long range quasi-periodic translational order of quasicrystals can render an interesting SLR to be explored experimentally by the technique introduced in Chapter 6 . This will provide more insights on the application of quasicrystalline plasmonic arrays in some areas requiring the enhancement of light-matter interaction.

\subsection{The potential application of Sierpinski carpet optical antennas}

The application of Sierpinski carpet optical antennas, though based on different designs, has actually been proposed or implemented for the enhancement of solar cell efficiency [103] and surface-enhanced Raman spectroscopy sensitivity [104]. However, these studies just used the fractal arrangement of metal micro- or nano-particles, without an understanding of why fractals work. That is to say, they lack a clear design rule to optimize the yield of the quantum efficiency of solar cells or Raman spectroscopy signals of molecules. The work in this thesis shows a route to compensate this inadequacy.

For other nanophotonic devices, resonant optical antennas have been applied in the photodetector [105] [106] and light-emitting diodes (LEDs) [107] design. The nonradiative decay of plasmons [108] in optical antennas generates energetic or 'hot' electron-hole pairs which are able to generate a photocurrent in the active semiconductor areas of a photodetector, resulting in the detection of light. This could enable low-cost silicon infrared photodetectors that may replace costly InGaAs detectors in the same spectral range. To apply the SLR of the Sierpinski carpet optical antenna on the photodetectors is yet unexplored. The difficulty comes from the effective contact between the active area of a photodetector with the Sierpinski carpet optical antenna. Using relatively larger-size fractal antennas in the photodetector design suffers the shrinkage of size of detection area, which is critical to the signal-to-noise ratio of a photodetector [19].

LEDs utilize the phosphor which absorbs a significant fraction of the light emitted by an electrically driven ultraviolet or blue LED and re-emits at a longer wavelength. The non-absorbed blue light mixes with the re-emitted light from the phosphor to provide white light. Most studies of solid-state 
lighting focus on the development of light emitters with high internal quantum efficiency (QE) [109] and light extraction mechanisms using photonic crystals [110] to meet the requirements for general illumination applications. The SLR of a periodic metal nanoparticle array has been shown to be capable of enhancing the performance of emitters (dyes) with QE close to 1 in the solid-state lighting. The SLR of a periodic metal nanoparticle array can further shape the angular pattern of emission, beaming most of the light into a defined direction with a very narrow angular range [107].

The SLR of a Sierpinski carpet optical antenna can also be considered to be adopted in solid-state lighting to enhance the performance of emitters. Different from the periodic metal nanoparticle array, the minimum distance between metal nanoparticles of Sierpinski carpet optical antenna can be shorter and still meet the resonance condition of the SLR. This increases the contact areas between the metal and the layer of emitters and therefore may increase the performance. 


\section{Bibliography}

[1] G. Marconi, "Wireless Telegraphic Communication: Nobel Lecture, 11 December 1909," Elsevier Publishing Company, Amsterdam, 1967.

[2] W. L. Stutzman and G. A. Thiele, Antenna Theory and Design, New York: John Wiley \& Sons, 2012.

[3] C. A. Pickover, The Math Book: From Pythagoras to the 57th Dimension, 250 Milestones in the History of Mathematics, Sterling Publishing Company, Inc, 2009.

[4] B. Mandelbrot, The fractal geometry of nature, Macmillan, 1983.

[5] W. Sierpinski, "Sur une courbe cantorienne qui contient une image biunivoque et continue de toute courbe donn'ee," C. R. Hebd. Seanc. Acad. Sci., vol. 162, p. 629, 1916.

[6] G. Volpe, G. Volpe and R. Quidant, "Fractal plasmonics: subdiffraction focusing and broadband spectral response by a Sierpinski nanocarpet," Optics Express, vol. 19, p. 3612, 2011.

[7] R. G. Hohlfeld and N. Cohen, "Self-similarity and the geometric requirements for frequency independence in antennae," Fractals, vol. 7, p. 79, 1999.

[8] L. Ukkonen, L. Sydänheimo and M. Kivikoski, "Read Range Performance Comparison of Compact Reader Antennas for a Handheld UHF RFID Reader," 2007 IEEE International Conference on RFID Gaylord Texan Resort, Grapevine, TX, USA, p. 63, 2007. 
[9] N. A. Saidatul, A. A. Azremi, R. B. Ahmad, P. J. Soh and F. Malek, "Multiband fractal planar inverted $\mathrm{F}$ antenna (F-PIFA) for mobile phone application," Progress in Electromagnetics Research B, vol. 14, p. 127, 2009.

[10] FRACTAL ANTENNA SYSTEMS INC., http://www.fractenna.com/.

[11] Fractus Technology, http://www.fractus.com/index.php/fractus/technology/.

[12] V. Radoni'c, K. Palmer, G. Stojanovi'c and C. Crnojevi'c-Bengin, "Flexible Sierpinski Carpet Fractal Antenna on a Hilbert Slot Patterned Ground," International Journal of Antennas and Propagation, p. 1, 2012.

[13] C. F. Bohren and D. R. Huffman, Absorption and scattering of light by small particles, New York: John Wiley \& Sons, 1983.

[14] K. A. Willets and R. P. Van Duyne, "Localized surface plasmon resonance spectroscopy and sensing," The Annual Review of Physical Chemistry, vol. 58, p. 267, 2007.

[15] Editorial article, "Surface plasmon resurrection," Nature Photonics, vol. 6, p. 707, NOVEMBER 2012.

[16] J. B. Pendry, "Negative Refraction Makes a Perfect Lens," Physical Review Letters, vol. 85, no. 18, p. 3966, October 2000.

[17] H. Raether, Surface plasmons on smooth and rough surfaces and on grating, Berlin: Springer, 1988.

[18] L. Novotny and Niek van Hulst, "Antennas for light," Nature Photonics, vol. 5, p. 83, February 2011.

[19] P. Bharadwaj, B. Deutsch and L. Novotny, "Optical antennas," Advances in Optics and Photonics, vol. 1, p. 438, 2009. 
[20] E. Abbe, "Beitrage zur theorie des mikroskops und der mikroskopischen wahrnehmung," Archiv F. Mikroskop. Anat, vol. 9, p. $413,1873$.

[21] Interview with Joachim Krenn, "Perspective on plasmonics," Nature Photonics, vol. 6, p. 714, November 2012.

[22] P. B. Johnson and R. W. Christy, "Optical constants of the noble metals," Physical Review B, p. 4370, 1972.

[23] L. Novotny and B. Hecht, Principles of Nano-Optics, Cambridge: Cambridge University Press, 2006.

[24] B. Hecht, H. Bielefeldt, L. Novotny, Y. Inouye and D. W. Pohl, "Local Excitation, Scattering, and Interference of Surface Plasmons," Physical Review Letters, vol. 77, p. 1889, 1996.

[25] A. Bouhelier, T. Huser, H. Tamaru, H. J. Guntherodt and D. W. Pohl, "Plasmon optics of structured silver films," Physical Review B, vol. 63, p. 155404, 2001.

[26] M. U. González, A. L. Stepanov, J.-C. Weeber, A. Hohenau, A. Dereux and R. Quidant, "Analysis of the angular acceptance of surface plasmon Bragg mirrors," Optics Letters, vol. 32, p. 2704, 2007.

[27] A. Norrman, T. Steala and A. T. Friberg, "Exact surface-plasmon polariton solutions at a lossy interface," Optics Letters, vol. 38, p. 1119, 2013.

[28] E. D. Palik, Handbook of optical constants of solids, Orlando, Florida: Academic press, 1991.

[29] S. Yang, H. Kobori, C. He, M. Lin, H. Chen, C. Li, M. Kanehara, T. Teranishi and S. Gwo, "Plasmon Hybridization in Individual Gold Nanocrystal Dimers: Direct Observation of Bright and Dark Modes," Nano Letters, p. 632, 2010. 
[30] D. E. Gomez, Z. Q. Teo, M. Altissimo, T. J. Davis, S. Earl and A. Roberts, "The Dark Side of Plasmonics," Nano Letters, p. 3722, 2013.

[31] F. Hao, E. M. Larsson, T. A. Ali, D. S. Sutherland and P. Nordlander, "Shedding light on dark plasmons in gold nanorings," Chemical Physics Letters, p. 262, 2008.

[32] S. J. Barrow, D. Rossouw, A. M. Funston, G. A. Botton and P. Mulvaney, "Mapping Bright and Dark Modes in Gold Nanoparticle Chains using Electron Energy Loss Spectroscopy," Nano Letters, p. 3799, 2014.

[33] F. Schertz, M. Schmelzeisen, R. Mohammadi, M. Kreiter, H.-J. Elmers and G. Schönhense, "Near Field of Strongly Coupled Plasmons: Uncovering Dark Modes," Nano Letters, p. 1885, 2012.

[34] W. Zhou and T. W. Odom, "Tunable subradiant lattice plasmons by out-of-plane dipolar interactions," Nature Nanotechnology, p. 423, 2011.

[35] U. Fano, "Effects of configuration interaction on intensities and phase shifts," Physical Reviews, p. 1866, 1961.

[36] B. Luk'yanchuk, N. I. Zheludev, S. A. Maier, N. J. Halas, P. Nordlander, H. Giessen and C. T. Chong, "The Fano resonance in plasmonic nanostructures and metamaterials," Nature Materials, p. 707, 2010.

[37] P. Tassin, L. Zhang, R. Zhao, A. Jain, T. Koschny and C. M. Soukoulis, "Electromagnetically Induced Transparency and Absorption in Metamaterials: The Radiating Two-Oscillator Model and Its Experimental Confirmation," Physical Review Letters, p. 187401, 2012.

[38] C. L. Alzar, M. A. Martinez and P. Nussensveig, "Claasical analog of electromagnetically induced transparency," American Journal of Physics, p. 37, 2002. 
[39] M. Hentschel, M. Saliba, R. Vogelgesang, H. Giessen, A. P. Alivisatos and N. Liu, "Transition from Isolated to Collective Modes in Plasmonic Oligomers," Nano Letters, p. 2721, 2010.

[40] B. Auguie' and W. L. Barnes, "Collective Resonances in Gold Nanoparticle Arrays," Physical Review Letters, vol. 101, p. 143902, 2008.

[41] E. M. Hicks, S. Zou, G. C. Schatz, K. G. Spears, R. P. Van Duyne, L. Gunnarsson, T. Rindzevicius, B. Kasemo and M. Ka, "Controlling Plasmon Line Shapes through Diffractive Coupling in Linear Arrays of Cylindrical Nanoparticles Fabricated by Electron Beam Lithography," Nano Letters, vol. 5, p. 1065, 2005.

[42] F. J. De Abajo, "Colloquium: Light scattering by particle and hole arrays," Review of Modern Physics, vol. 79, p. 1267, 2007.

[43] Y. Chu, E. Schonbrun, T. Yang and K. B. Crozier, "Experimental observation of narrow surface plasmon resonances in gold nanoparticle arrays," Applied Physics Letters, vol. 93, p. 181108, 2008.

[44] V. G. Kravets, F. Schedin and A. N. Grigorenko, "Extremely narrow plasmon resonances based on diffraction coupling of localized plasmons in arrays of metallic nanoparticles," Physical Review Letters, vol. 101, p. 87403, 2008.

[45] G. Vecchi, V. Giannini and J. Gómez Rivas, "Shaping the Fluorescent Emission by Lattice Resonances in Plasmonic Crystals of Nanoantennas," Physical Review Letters, vol. 102, p. 146807, 2009.

[46] J. S. Huang, V. Callegari, P. Geisler, E. Bruning, J. Kern, J. E. Prangsma, X. Wu, T. Feichtner, J. Ziegler, P. Weinmann, M. Kamp, A. Forchel, P. Biagioni, U. Sennhauser and B. Hecht, "Atomically flat single-crystalline gold nanostructures for plasmonic nanocircuitry," Nature Communications, vol. 1, p. 1, 2010.

[47] C. Kan, X. Zhu and G. Wang, "Single-crystalline gold microplates: 
synthesis, characterization, and thermal stability," The Journal of Physical Chemistry B, vol. 110, p. 4651, 2006.

[48] R. Geurts and S. Reyntjens, NOVA Nanolab Training Course, Eindhoven: FEI Electron Optics B.V., 2004.

[49] W. Qian, X. Huang, B. Kang and M. A. El-Sayed, "Dark-field light scattering imaging of living cancer cell component from birth through division using bioconjugated gold nanoprobes," Journal of Biomedical Optics, vol. 154, p. 46025, 2002.

[50] J. J. Mock, M. Barbic, D. R. Smith, D. A. Schultz and S. Schultz, "Shape effects in plasmon resonance of individual colloidal silver Nanoparticles," Journal of Chemical Physics, vol. 116, p. 6755, 2002.

[51] M. Hu, C. Novo, A. Funston, H. Wang, H. Staleva, S. Zou, P. Mulvaney, Y. Xia and G. V. Hartland, "Dark-field microscopy studies of single metal nanoparticles: understanding the factors that influence the linewidth of the localized surface plasmon resonance," Journal of Materials Chemistry, vol. 18, p. 1949, 2008.

[52] C. L. Nehl, N. K. Grady, G. P. Goodrich, F. Tam, N. J. Halas and J. H. Hafner, "Scattering Spectra of Single Gold Nanoshells," Nano Letters, p. 2355, 2004.

[53] M. W. Knight, J. Fan, F. Capasso and N. J. Halas, "Influence of excitation and collection geometry on the dark field spectra of individual plasmonic nanostructures," Optics Express, p. 2579, 2010.

[54] T-L. Chen, D. J. Dikken, J. C. Prangsma, F. Segerink and J. L. Herek, "Characterization of Sierpinski carpet optical antenna at visible and near-infrared wavelengths," New Journal of Physics, vol. 16, p. 093024, 2014.

[55] P. Ghenuche, S. Cherukulappurath, T. H. Taminiau, N. F. van Hulst and R. Quidant, "Spectroscopic mode mapping of resonant plasmon nanoantennas," Physical Review Letters, vol. 101, p. 116805, 2008. 
[56] P. J. Schuck, D. P. Fromm, A. Sundaramurthy, G. S. Kino and W. E. Moerner, "Improving the mismatch between light and nanoscale objects with gold bowtie nanoantennas," Physical Review Letters, vol. 94, p. 17402, 2005.

[57] S. Viarbitskaya, A. Teulle, R. Marty, J. Sharma, C. Girard, A. Arbouet and E. Dujardin, "Tailoring and imaging the plasmonic local density of states in crystalline nanoprisms," Nature Materials, vol. 12, p. 426, 2013.

[58] M. R. Beversluis, A. Bouhelier and L. Novotny, "Continuum generation from single gold nanostructures through near-field mediated intraband transitions," Physical Review B, vol. 68, p. 115433, 2003.

[59] D. W. Pohl, W. Denk and M. Lanz, "Optical stethoscopy: Image recording with resolution $\lambda / 20, "$ Applied Physics Letters, vol. 44, p. $651,1994$.

[60] V. A. Markel, "Antisymmetrical optical states," Journal of the Optical Society of America B, vol. 12, p. 1783, 1995.

[61] K. H. Fung and C. T. Chan, "Plasmonic modes in periodic metal nanoparticle chains: a direct dynamic eigenmode analysis," Optics Letters, vol. 32, p. 973, 2007.

[62] V. L. Lokea and M. P. Mengc", "Discrete-dipole approximation with surface interaction: Computational toolbox for MATLAB," Journal of Quantitative Spectroscopy \& Radiative Transfer, vol. 112, p. 1711, 2011.

[63] B. T. Draine and P. J. Flatau, "Discrete-dipole approximation for scattering calculations," Journal of Optical Society of America A, vol. 11, p. 1491, 1994.

[64] W. T. Doyle, "Optical properties of a suspension of metal spheres," Physical Review B, vol. 39, p. 9852, 1989. 
[65] J.-W. Dong, K.-H. Fung, C.-T. Chan and H.-Z. Wang, "Localization characteristics of two-dimensional quasicrystals consisting of metal nanoparticles," Physical Review B, vol. 80, p. 155118, 2009.

[66] C. Forestiere, G. Miano, G. Rubinacci and L. Dal Negro, "Role of aperiodic order in the spectral, localization, and scaling properties of plasmon modes for the design of nanoparticle arrays," Physical Review $B$, vol. 79, p. 85404, 2009.

[67] M. I. Stockman, S. V. Faleev and D. J. Bergman, "Localization versus delocalization of surface plasmons in nanosystems: can one state have both characteristics?," Physical Review Letters, vol. 108, p. 77404, 2001.

[68] A. Gopinath, S. V. Boriskina, N.-N. Feng, B. M. Reinhard and L. Dal Negro, "Photonic-plasmonic scattering resonances in deterministic aperiodic structures," Nano Letters, vol. 8, p. 2423, 2008.

[69] L. Dal Negro and S. V. Boriskina, "Deterministic aperiodic nanostructures for photonics and plasmonics applications," Lasers \& Photonics Reviews, vol. 6, p. 178, 2012.

[70] R. Dallapiccola, A. Gopinath, F. Stellacci and L. Dal Negro, "Quasiperiodic distribution of plasmon modes in two-dimensional Fibonacci arrays of metal nanoparticles," Optics Express, vol. 16, p. 5544, 2008.

[71] V. E. Ferry, M. A. Verschuuren, C. van Lare, R. J. Walters, R. E. Schropp, H. A. Atwater and A. Polman, "Optimized spatial correlations for broadband light trapping in ultra-thin a-Si:H solar cells," Nano Letters, vol. 11, p. 4239, 2011.

[72] A. Hohenau, J. R. Krenn, F. J. Garcia-Vidal, S. G. Rodrigo, L. MartinMoreno, J. Beermann and S. I. Bozhevolnyi, "Spectroscopy and nonlinear microscopy of gold nanoparticle arrays on gold films," Physical Review B, vol. 35, p. 133, 2010. 
[73] C. Forestiere, M. Donelli, G. F. Walsh, E. Zeni, G. Miano and L. Dal Negro, "Particle-swarm optimization of broadband nanoplasmonic arrays," Optics Letters, vol. 35, p. 133, 2010.

[74] M. Donelli, "Design of broadband metal nanosphere antenna arrays with a hybrid evolutionary algorithm," Optics Letters, vol. 38, p. 401, 2013.

[75] A. Hessel and A. A. Oliner, "A New Theory of Wood's Anomalies on Optical Gratings," Applied Optics, vol. 4, p. 1275, 1965.

[76] U. Fano, "The Theory of Anomalous Diffraction Gratings and of Quasi-Stationary Waves on Metallic Surfaces (Sommerfeld's Waves)," Journal of Optical Society of America A, vol. 31, p. 213, 1941.

[77] M. Sarrazin, J.-P. Vigneron and J.-M. Vigoureux, "Role of Wood anomalies in optical properties of thin metallic films with a bidimensional array of subwavelength holes," Physical Review B, vol. 67, p. 85415, 2003.

[78] M. Segev, M. Soljačić and J. M. Dudley, "Fractal optics and beyond," Nature Photonics, vol. 6, p. 209, 2012.

[79] "2D quasiperiodic plasmonic crystals," Scientific Reports, vol. 2, p. $681,2012$.

[80] C. Forestiere, G. F. Walsh, G. Miano and L. Dal Negro, "Nanoplasmonics of prime number arrays," Optics Express, vol. 17, p. 24288, 2009.

[81] J. W. Goodman, Introduction to Fourier Optics, New York: MaGrawHoll, 1968.

[82] A. Drezet, A. Hohenau, A. L. Stepanov, H. Ditlbacher, B. Steinberger, N. Galler, F. R. Aussenegg, A. Leitner and J. R. Krenn, "How to erase surface plasmon fringes," Applied Physics Letters, vol. 89, p. 91117, 
2006.

[83] B. Hopkins, A. N. Poddubny, A. E. Miroshnichenko and Y. S. Kivshar, "Revisiting the physics of Fano resonances for nanoparticle oligomers," Physical Review A, vol. 88, p. 53819, 2013.

[84] N. I. Zheludev, S. A. Maier, N. J. Halas, P. Nordlander, H. Giessen and C.-T. Chong, "The Fano resonance in plasmonic nanostructures and metamaterials," Nature Materials, vol. 9, p. 707, 2010.

[85] J. A. Fan, C. H. Wu, K. Bao, R. Bardhan, N. J. Halas, V. N. Manoharan, P. Nordlander, G. Shvets and F. Capasso, "Self-Assembled Plasmonic Nanoparticle Clusters," Science, vol. 328, p. 1135, 2010.

[86] M. Frimmer, T. Coenen and F. Koenderink, "Signature of a Fano Resonance in a Plasmonic Metamolecule's Local Density of Optical States," Physical Review Letters, vol. 108, p. 77404, 2012.

[87] S. R. Rodriguez, A. Abass, B. Maes, O. T. Janssen, G. Vecchi and J. Go'mez Rivas, "Coupling Bright and Dark Plasmonic Lattice Resonances," Physical Review X, vol. 1, p. 21019, 2011.

[88] S. A. Maier, "Plasmonics: The benefits of darkness," Nature Materials, vol. 8, p. 699, 2009.

[89] J. N. Anker, W. P. Hall, O. Lyanders, N. C. Shah, J. Zhao and R. P. Van Duyne, "Biosensing with plasmonic nanosensors," Nature Materials, vol. 7, p. 442, 2008.

[90] C. Bauer, G. Kobiela and H. Giessen, "Optical properties of twodimensional quasicrystalline plasmonic arrays," Physical Review B, vol. 84, p. 193104, 2011.

[91] C. Bauer, G. Kobiela and H. Giessen, "2D quasiperiodic plasmonic crystals," Scientific Reports, vol. 2, p. 681, 2012. 
[92] S. Kasture, A. P. Ravishankar, V. J. Yallapragada, R. Patil, N. V. Valappil, G. Mulay and V. G. Achanta, "Plasmonic quasicrystals with broadband transmission enhancement," Scientific Reports, vol. 4, p. 1, 2014.

[93] A. Gopinath, S. V. Boriskina, S. Yerci, R. Li and L. Dal Negro, "Enhancement of the 1.54 um Er3+ emission from quasiperiodic," Applied Physics Letters, vol. 96, p. 71113, 2010.

[94] C. Forestiere, G. Miano, . S. V. Boriskina and L. Dal Negro, "The role of nanoparticle shapes and deterministic aperiodicity for the design of nanoplasmonic arrays," Optics Express, vol. 17, p. 9648, 2009.

[95] "Crystals of golden proportions," Technical Report, The Royal Swedish Academy, 2011.

[96] D. Shechtman, I. Blech, D. Gratias and J. W. Cahn, "Metallic Phase with Long-Range Orientational Order and No Translational Symmetry," Physical Review Letters, vol. 53, p. 1951, 1984.

[97] L. Bindi and P. J. Steinhardt, "The quest for forbidden crystals," Mineralogical Magazine, vol. 78, p. 467, 2014.

[98] D. Levine and P. J. Steinhardt, "Quasicrystals. I. Definition and structure," Physical Review B, vol. 34, p. 596, 1986.

[99] J.-W. Dong, K.-H. Fung, C.-T. Chan and H.-Z. Wang, "Localization characteristics of two-dimensional quasicrystals consisting of metal nanoparticles," Physical Review B, vol. 80, p. 155118, 2009.

[100] Z.-L. Deng, Z.-H. Li, J.-W. Dong and H.-Z. Wang, "In-Plane Plasmonic Modes in a Quasicrystalline Array of Metal Nanoparticles," Plasmonics, vol. 6, p. 507, 2011.

[101] C. Bauer and H. Giessen, "Light harvesting enhancement in solar cells with quasicrystalline plasmonic structures," Optics Express, vol. 21, p. 
A363, 2013.

[102] R. Verre, T. J. Antosiewicz, M. Svedendahl, K. Lodewijks, T. Shegai and M. Ka"1l, "Quasi-isotropic Surface Plasmon Polariton Generation through Near-Field Coupling to a Penrose Pattern of Silver Nanoparticles," ACS Nano, vol. 8, p. 9286, 2014.

[103] L.-H. Zhu, M.-R. Shao, R.-W. Peng, R.-H. Fan, X.-R. Huang and M. Wang, "Broadband absorption and efficiency enhancement of an ultrathin silicon solar cell with a plasmonic fractal," Optics Express, vol. 21, p. 313, 2013.

[104] K.-H. Hsu, J. H. Back, K.-H. Fung, P. M. Ferreira, M. Shim and N. X. Fang, "SERS EM field enhancement study through fast Raman mapping of Sierpinski carpet arrays," Journal of Raman Spectroscopy, vol. 41, p. 1124, 2010.

[105] M. W. Knight, H. Sobhani, P. Nordlander and N. J. Halas, "Photodetection with Active Optical Antennas," Science, vol. 332, p. 702, 2011.

[106] L. TANG, S. E. KOCABAS, S. LATIF, A. K. OKYAY, D.-S. LYGAGNON, K. C. SARASWAT and D. A. B. MILLER, "Nanometrescale germanium photodetector enhanced by a near-infrared dipole antenna," Nature Photonics, vol. 226, p. 2, 2008.

[107] G. Lozano, D. J. Louwers, S. R. Rodri'guez, S. Murai, O. T. Jansen, M. A. Verschuuren and J. G. Rivas, "Plasmonics for solid-state lighting: enhanced excitation and directional emission of highly efficient light sources," Light: Science \& Applications, vol. 2, p. 1, 2013. 


\section{List of publications}

This thesis is based on the following publications:

- Characterization of Sierpinski carpet optical antenna at visible and near-infrared wavelengths, Ting Lee Chen, Dirk Jan Dikken, Jord C. Prangsma, Frans Segerink and Jennifer L. Herek, New J. Phys. 16 093024 (2014). (Chapter 5)

- Observation of a surface lattice resonance in a fractal arrangement of gold nanoparticles, Ting Lee Chen, Jord C. Prangsma, Frans Segerink, Dirk Jan Dikken and Jennifer L. Herek, in preparation. (Chapter 6)

- Analysis of plasmonic eigenmodes of Sierpinski Carpet Optical Antenna by Eigen-Decomposition Method, Ting Lee Chen, Jord C. Prangsma and Jennifer L. Herek, in preparation. (Chapter 4)

Other publication by the author:

- Investigation of adaptive laser pulse shaping by direct estimation of group delay profile, Di Yang, Daan P. Sprünken, Alexander C.W. van Rhijn, Janne Savolainen, Ting Lee Chen, Herman L. Offerhaus, Jennifer L. Herek and Aliakbar Jafarpour, Optics Communications 284, 2748 (2011) 


\section{Summary}

In this thesis, we describe experimental and theoretical investigations of the properties of a fractal optical antenna-the Sierpinski carpet optical antenna. Fractal optical antennas are inspired by fractal antennas designed in radio frequency (RF) region. Shrinking the size of fractal optical antennas from fractal antennas in RF regions by a factor of $10^{-5}$ arises challenges of fabrication, characterization and modelling their response to incident light. The comparison between optical antennas with the Sierpinski carpet geometry and other geometries is also an interesting topic. Generally speaking, this thesis covers the fabrication, the theoretical investigations of the properties of eigenmodes and the characterization of Sierpinski carpet optical antennas by optical techniques. Due to the versatile application of optical antennas, the generalization and application of the theoretical and experimental techniques to other plasmonic arrays, or even the application of the Sierpinski carpet optical antenna itself to other areas of light-matter interaction, can be expected.

In Chapter 2, the fundamental concepts of surface plasmon polaritons (SPPs) and optical antennas have been introduced. The excitation condition of SPPs and localized surface plasmons (LSPs) in metal nanoparticles is presented. Resonant optical antennas utilize LSPs to capture or emit light waves and therefore transform propagating lights into localized electromagnetic fields or vice versa. The concepts of super- (bright) and sub radiant (dark) plasmonic modes are introduced. The coupling between superand sub-radiant plasmonic modes gives rise to the Fano resonance in plasmonic nanostructures, which causes the Fano-dip in scattering spectra of plasmonic nanostructures. This is further discussed in Chapter 6.

In Chapter 3, the concept of fractal geometry and the adoption thereof in fractal antennas for broadband wireless communications is introduced. The idea of the Sierpinski carpet geometry is applied to the optical antenna design. Sierpinski carpet optical antennas are directly carved on single crystalline gold flakes by FIB milling. The schematics and working principles of optical 
diagnostic tools are introduced: the white-light dark field microscopy, the two photon photoluminescence (TPPL) microscopy and the back focal plane microscopy. The white-light dark field microscopy is used for the determination of the resonant wavelength of a plasmonic nanostructure, and the application of it can be found in Chapters 5 and 6. The TPPL microscopy is used for the measurement of near-field intensity enhancement of a plasmonic nanostructure, and the application of it can be found in Chapter 5. The back focal plane microscopy is used to map the plasmonic modes of a plasmonic nanostructure on the back focal plane (or Fourier plane) of an objective, and the application of it is discussed in Chapter 6 .

In Chapter 4, the influence of the self-similarity on the eigenmodes of the Sierpinski carpet optical antenna has been investigated by the eigendecomposition method. The concepts of eigenpolarizability and participation ratio (PR) are introduced. The eigenpolarizability spectrum of out-of-plane eigenmodes has a super- and a sub-radiant peak. The spectral positions of these two peaks remain unchanged as the order of the Sierpinski carpet optical antenna increases. For the in-plane eigenmodes, there does not exist such a clear super- or sub-radiant peak. The self-similarity in out-of-plane and in-plane eigenmodes is illustrated by the plots of dipole moment structures of 1 st - 3rd order Sierpinski carpet optical antennas. The super- and sub-radiance of eigenmodes are investigated by their Fourier amplitudes. Their localization property is indicated by PR values of eigenmodes. This helps us to understand how fractal geometry influences the eigenpolarizability of eigenmodes as the order increases.

In Chapter 5, three different morphologies of optical antennas are investigated: the Sierpinski carpet, pseudo-random and periodic morphologies. The far-field scattering property and near-field intensity enhancement of three morphologies are investigated by the white-light dark field microscopy and the TPPL microscopy, respectively. For the scattering response, we find all three morphologies give broadband response. However, the near-field intensity measured by the TPPL microscopy shows that the Sierpinski carpet has the highest intensity in a narrow spectral range.

In Chapter 6, a surface lattice resonance (SLR) of a Sierpinski carpet optical antenna is visualized by the back focal plane microscopy. This SLR comes from the coupling between a dominant subradiant eigenmode and inplane diffracted waves. This dominant subradiant eigenmode is calculated by 
the eigen-decomposition method. This SLR generates a Fano-dip in the scattering spectrum. The experimental results are supported and explained by the finite difference time domain and coupled dipole approximation methods.

Finally, Chapter 7 explores potential directions for future research and possible applications. 


\section{Acknowledgement}

I thank God and my Lord Jesus for His helps and graces through these years in Netherland. In my need, He has always been there, comforting me with His great faithfulness and unfailing love. May glory and praise be to God and His precious son, my dearest Lord Jesus forever.

For my $\mathrm{PhD}$ works in this thesis, I can't describe how much I am indebted to my supervisor Prof. Jennifer Herek: for accepting me the as a $\mathrm{PhD}$ student in the Optical Sciences group; for her tolerance and patience on allowing me to explore some research areas but without results for quite a long time; for generously encouraging me to attend many nice conferences and an unforgettable summer school in Erice, Italy. Especially I have to express my gratitude on her strict and highly-demanding attitude toward me during my $\mathrm{PhD}$ time to help me focusing on the right track and push this thesis, as she said, a piece of work we are proud of.

Many people in our group contributed their talents and skills to make this thesis possible. I have to give my thanks to Jeroen and Frans, the best scientific engineers I have ever seen. Thank Jeroen for helping me on the construction of my setups and program development; Thank Frans for fabricating high quality samples for my experiments; Thank Postdoc Jord for his ingenius suggestions on my experiments and manuscripts writing. Thank Dirk Jan for building a nice setup to let me use it. Thank Annemarie for suggestions on my thesis writing and schedule planning. Thank Sonia for allowing me to use the Lumerical software and Mustafa for teaching me how to use it. Though not directly involved in my thesis work, Herman's brilliant physics and talents inspire me. Thanks to him, I could hear some fancy ideas like mosquito laser canon, Raman spectroscopy on the sex drug inspection (Essence of Kangaroo?) and lots of bee's knowledge - not practical but terribly funny.

Many friendly and smart people in group enrich my life and should be mentioned here: Joris, Jorick, Sean, Bart, Sven, David, Gerwin, Qing, Rick, 
Sergio, Yean-sheng, Jinfeng, Lourens and Cedric. For some people not in group now: Eric, Martin, Jincy, Di, Ali, Alexander, Jesse, Divya, Bente, Oya, Andew, Bob and Peter. Thank all of you for giving me so much fun and supports during this period of time in the Optical Sciences group.

At last but not the least, I have to thank Prof. Rudolf Bratschitsch, Prof. Kobus Kuipers, Prof. Han Gardiniers, Prof. Klaus Boller and Dr. Mark Bentum for the approval of this thesis. It's really my great pleasure to have them as my thesis committee members.

在此要向我親愛的母親張桂金, 兩位姐姐陳齊陳渝, 在我追求博士學位 期間, 對我的支持與愛護跟為我擔憂, 表達深深的感謝。願主賜福給我 們一家。 


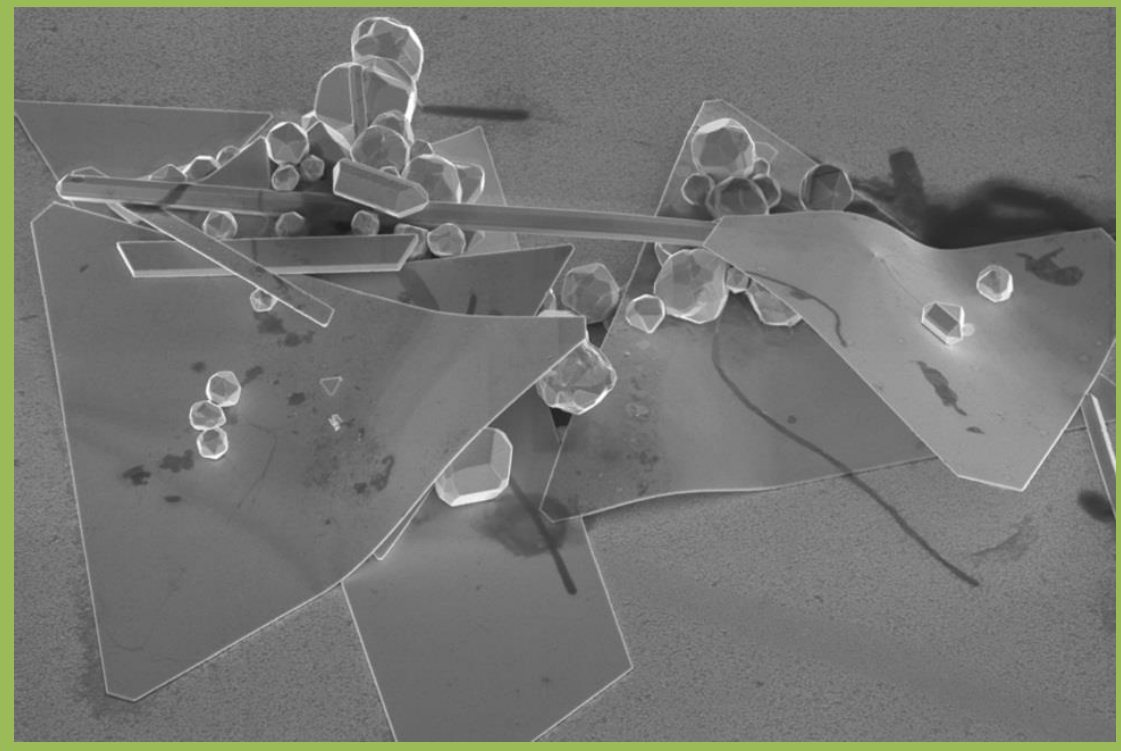

ISBN: 978-90-365-3894-7 